\title{
Statistical Exploratory Analysis of Genetic Algorithms
}

\author{
THIS THESIS IS \\ PRESENTED TO THE \\ School of Computer Science \& Software Engineering \\ FOR THE DEGREE OF \\ Doctor of Philosophy \\ $\mathrm{OF}$ \\ The University of Western Australia
}

By

Andrew Simon Timothy Czarn

February 2008 

(C) Copyright 2008

by

Andrew Simon Timothy Czarn 


\section{Statistical Exploratory Analysis of Genetic Algorithms}

enetic algorithms (GAs) have been extensively used and studied in com-
puter science, yet there is no generally accepted methodology for exploring which parameters significantly affect performance, whether there is any interaction between parameters and how performance varies with respect to changes in parameters.

This thesis presents a rigorous yet practical statistical methodology for the exploratory study of GAs. This methodology addresses the issues of experimental design, blocking, power and response curve analysis. It details how statistical analysis may assist the investigator along the exploratory pathway.

The statistical methodology is demonstrated in this thesis using a number of case studies with a classical genetic algorithm with one-point crossover and bit-replacement mutation. In doing so we answer a number of questions about the relationship between the performance of the GA and the operators and encoding used. The methodology is suitable, however, to be applied to other adaptive optimization algorithms not treated in this thesis.

In the first instance, as an initial demonstration of our methodology, we describe case studies using four standard test functions. It is found that the effect upon performance of crossover is predominantly linear while the effect of mutation is predominantly quadratic. Higher order effects are noted but contribute less to 
overall behaviour. In the case of crossover both positive and negative gradients are found which suggests using rates as high as possible for some problems while possibly excluding it for others. For mutation, optimal rates appear higher than earlier recommendations while supporting more recent work. The significance of interaction and the best values for crossover and mutation are problem specific.

Secondly, an original benchmark test function is developed, FNn, and it is demonstrated that as the test function increases in modality the interaction between crossover and mutation becomes statistically significant. The effect of interaction is striking when examining response curves, which illustrate distinct inflection. It is conjectured that for highly modal functions the possibility of interaction between crossover and mutation must be considered. Moreover, the practical implication of interaction is that when attempting to fine tune a GA on highly modal problems the optimal rates for crossover and mutation cannot be obtained independently. All combinations of crossover and mutation, within given starting ranges, must be investigated in order to allow for the interaction effect.

Thirdly, an important issue in GAs is the relationship between the difficulty of a problem and the choice of encoding. Two questions remain unanswered: is there a statistically demonstrable relationship between the difficulty of a problem and the choice of encoding, and, if so, what is the actual mechanism by which this occurs. In this thesis we use components of the statistical methodology developed to demonstrate that the choice of encoding has a real effect upon the difficulty of a problem. This is illustrated by showing how the use of Gray codes impedes the performance on a lower modality test function compared with a higher modality test function. Computer animation is then used to illustrate the actual mechanism by which this occurs.

Fourthly, the traditional concept of a GA is that of selection, crossover and mutation. However, a limited amount of data from the literature has suggested that the niche for the beneficial effect of crossover upon GA performance may be smaller than has traditionally been held. Based upon previous results on not-linear-separable 
problems an exploration is made by comparing two test problem suites, one comprising non-rotated functions and the other comprising the same functions rotated by 45 degrees in the solution space rendering them not-linear-separable.

It is shown that for the difficult rotated functions the crossover operator is detrimental to the performance of the GA. It is conjectured that what makes a problem difficult for the GA is complex and involves factors such as the degree of optimization at local minima due to crossover, the bias associated with the mutation operator and the Hamming Distances present in the individual problems due to the encoding.

Furthermore, the GA was tested on a real world landscape minimization problem to see if the results obtained would match those from the difficult rotated functions. It is demonstrated that they match and that the features which make certain of the test functions difficult are also present in the real world problem.

Overall, the proposed methodology is found to be an effective tool for revealing relationships between a randomized optimization algorithm and its encoding and parameters that are difficult to establish from more $a d-h o c$ experimental studies alone. 


\section{Preface}

graphic details of the works and where they appear in the thesis are set out
graphic published work which has been co-authored. The bibliobelow.

1. Chapter 2: A.S.T. Czarn, C. MacNish, K. Vijayan B. Turlach, and R. Gupta. Statistical exploratory analysis of genetic algorithms. IEEE Transactions on Evolutionary Computation. Pages 405-421. Number 4, Volume 8, August, IEEE Press, 2004.

This paper was nominated for the IEEE Best Paper Award.

2. Chapter 3: A.S.T. Czarn, C. MacNish, K. Vijayan and B. Turlach. Statistical exploratory analysis of genetic algorithms: the importance of interaction. Proceedings of the 2004 IEEE Congress on Evolutionary Computation (CEC 2004). Pages 2288-2295. June, IEEE Press, 2004.

3. Chapter 4: A.S.T. Czarn, C. MacNish, K. Vijayan and B. Turlach. Statistical exploratory analysis of genetic algorithms: the influence of Gray Codes upon the difficulty of a problem. Proceedings of the 17th Australian Joint Conference on Artificial Intelligence (AI 2004). Pages 1246-1252. LNAI 3339, December, Springer, 2004. 
4. Chapter 5: A.S.T. Czarn, C. MacNish, K. Vijayan and B. Turlach. The Detrimentality of Crossover. Proceedings of the 20th Australian Joint Conference on Artificial Intelligence (AI 2007). Pages 632-636. LNAI 4830, December, Springer, 2007.

Though a number of authors are present on each individual publication, the authors acted in a supervisory capacity only. It is the $\mathrm{PhD}$ candidate that has been responsible for the work presented in this thesis, as signed by the $\mathrm{PhD}$ candidate and supervisors below:

Andrew Czarn

Cara MacNish

Kaipillil Vijayan 


\section{Acknowledgements}

ara MacNish was instrumental in this part of my academic career. I respect $\checkmark$ Cara as an individual of significant intellect and I humbly offer Cara my profound thanks and appreciation.

I should like to also thank Kaipillil Vijayan for the honour of allowing me to complete a PhD thesis under his supervision. I thank Kaipillil Vijayan also for his personal support and assistance.

I could not have completed this doctorate without the collaboration of Berwin Turlach to whom I also owe my profound thanks and appreciation.

During this doctorate I contracted a life-threatening illness which left sustained problems with my health. However, with the support of an exceptional team of health professionals I have been able to complete the present work. Thus, my many thanks go to Simon Byrne, Philip Melling, Avonia Donnellan, Leanne Dusz, John Kennedy, Richard O'Regan, Brian Russell and Andrew Klimaitis. However, special thanks must go to John Martin, one of the most eminent people I will ever have the pleasure to meet in this life.

In conclusion, I would like to thank my parents, Margot and Mark. I dedicate this doctorate to my mother and to the memory of my late father who passed away during its completion. May God bless these two people to whom I owe so much. 


\section{Contents}

$\begin{array}{ll}\text { Preface } & \text { ix }\end{array}$

Acknowledgements $\quad$ xi

1 Introduction $\quad 1$

1.1 Genetic Algorithms . . . . . . . . . . . . . . . . 2

1.2 Thesis Structure . . . . . . . . . . . . . . . . . . 3

$\begin{array}{lll}2 & \text { Statistical Methodology } & 7\end{array}$

2.1 Background ......................... 8

2.2 Non-Statistical Exploratory Analysis . . . . . . . . . . . . . . . . 9

2.3 Statistical Exploratory Analysis . . . . . . . . . . . . . . . 11

2.4 Methods . . . . . . . . . . . . . . . . . . . . . 13

2.4.1 Choice of Standard Test Functions . . . . . . . . . . . . 13

2.4 .2 Implementation of the GA . . . . . . . . . . 14

2.4.3 Experimental Design and Statistical Test . . . . . . . . . . 14

2.4.4 Choice of Level of Significance . . . . . . . . . . . . . . . 20 
2.4.5 Level of Significance for Orthogonal Simultaneous Multiple Comparisons ..................... 20

2.4.6 Power . . . . . . . . . . . . . . . . . . . . 21

2.4.7 Simultaneous Confidence Intervals for the Plotted Response

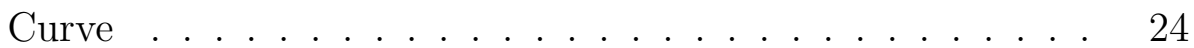

2.4.8 Pooled Analysis Design . . . . . . . . . . . . . . . . . . 24

2.4.9 Estimates of Best Values for Parameters . . . . . . . . . 25

2.4.10 Workup Procedures to Ensure a Balanced ANOVA Design . 25

2.5 Results . . . . . . . . . . . . . . . . . . . . 27

2.5.1 Exploratory Analysis of Test Function F1 . . . . . . . . 27

2.5.2 Exploratory Analysis of Test Function F3 . . . . . . . . 34

2.5.3 Exploratory Analysis of Test Function F2 . . . . . . . . . 36

2.5.4 Exploratory Analysis of Test Function F6 . . . . . . . . . 38

2.6 Discussion . . . . . . . . . . . . . . . . . . . . . . . 41

3 The Importance of Interaction $\quad 45$

3.1 Background ........................... 46

3.2 Methods . . . . . . . . . . . . . . . . . . . 47

3.2.1 Test Functions . . . . . . . . . . . . . . . . . . . . . . . . . 47

3.2 .2 Power . . . . . . . . . . . . . . . . . . . . . 48

3.3 Results . . . . . . . . . . . . . . . . . . . . . . . 49

3.3.1 ANOVA Analysis of Test Functions . . . . . . . . . . . 49

3.3.2 Polynomial Regression Analysis of Test Functions . . . . . . 49

3.3.3 Polynomial Regression Graphs of Test Functions FN5, FN6 51

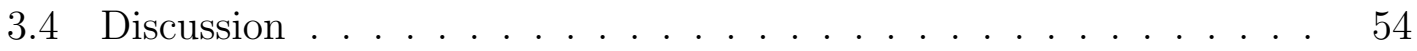


4.1 Background ......................... 57

4.2 Methods . . . . . . . . . . . . . . . . . . . . 58

4.2 .1 Test Functions . . . . . . . . . . . . . . . . 59

4.2 .2 Animation Analysis . . . . . . . . . . . . . . . . . . . . . 59

4.3 Results . . . . . . . . . . . . . . . . . . . 60

4.3.1 Response Curve Analysis of FN3 and FN4 . . . . . . . 60

4.3.2 Dot Diagram Analysis of FN3 and FN4 . . . . . . . . . 60

4.3.3 Dot Diagram Analysis of One Dimensional Projections . . . 61

4.3.4 Animation Analysis of $F N 3_{1 D}$ and $F N 41 D \ldots \ldots 63$

4.3.5 Hamming Distances for $F N_{1 D}$ and $F N_{41 D} \ldots \ldots . . . .65$

4.4 Discussion . . . . . . . . . . . . . . . . . . . . . . 67

5 The Detrimentality of Crossover $\quad 69$

5.1 Background . . . . . . . . . . . . . . . . . . 70

5.2 Observations from Earlier Work . . . . . . . . . . . . . . . 72

5.3 Methods . . . . . . . . . . . . . . . . . . . . . 74

5.3.1 Motivation for our Test Functions . . . . . . . . . . . . . 74

5.3 .2 Power ........................ 75

5.3.3 Estimates of Optimal Values for Crossover and Mutation . . 76

5.4 Results . . . . . . . . . . . . . . . . 76

5.4.1 Exploratory Analysis of Test Functions FN1 to FN6 . . . . 76

5.4.2 Exploratory Analysis of test functions FN1R45 to FN6R45 77

5.5 Factors Affecting the Detrimentality of Crossover . . . . . . . . . 78

5.5.1 Optimization Occurring at Local Minima due to Crossover . 78 
5.5.2 Bias Associated with the Mutation Operator . . . . . . . 81

5.5.3 Relationship between Gray Encoding and the Solution Space 83

5.6 Extending the Results to Difficult Practical Problems . . . . . . . . 87

5.7 Discussion . . . . . . . . . . . . . . . . . . . . . . . . . 89

6 General Conclusions and Future Research $\quad 93$

6.1 Statistical Methodology . . . . . . . . . . . . . . . 93

6.2 The Importance of Interaction . . . . . . . . . . . . . . . . . . . 95

6.3 The Influence of Gray Encoding . . . . . . . . . . . . . . . . . 96

6.4 The Detrimentality of Crossover . . . . . . . . . . . . . . 96

6.5 Future Research . . . . . . . . . . . . . . . . . . 97

$\begin{array}{lr}\text { Bibliography } & 99\end{array}$

$\begin{array}{ll}\text { Appendices } & 105\end{array}$

$\begin{array}{ll}\text { A F1, F3, F2 and F6 } & 105\end{array}$

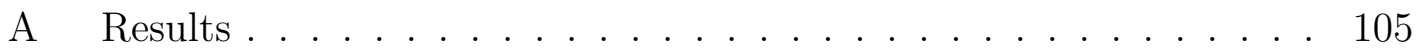

$\begin{array}{ll}\text { B FN1 to FN6 } & 125\end{array}$

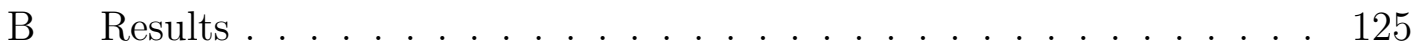

C FN1R45 to FN6R45 and Landscape_20_101 133

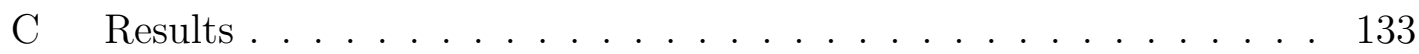




\section{List of Tables}

1 Genetics and GA Terminology . . . . . . . . . . . . 2

2 Recommendations for basic parameter settings . . . . . . . . . . 10

$3 \quad$ Recommendations for basic parameter settings using statistics. . . . 11

4 Details of the GA . . . . . . . . . . . . . . . 15

$5 \quad$ Creating a data-file from replicates of blocks. . . . . . . . . . . 16

$6 \quad$ Final ranges for crossover and mutation. . . . . . . . . . . . . . 27

7 F1-ANOVA of 100 replicates. . . . . . . . . . . . . 28

8 F1-ANOVA of 500 replicates. . . . . . . . . . . . 30

9 F1-Pooled ANOVA analysis. . . . . . . . . . . . . . . 32

10 F1-Overall results for crossover and mutation. . . . . . . . . . . . . 34

11 F3-Pooled ANOVA analysis. . . . . . . . . . . . . . . 34

$12 \quad$ F3-Overall results for crossover and mutation. . . . . . . . . . . 35

13 F2-Pooled ANOVA analysis. . . . . . . . . . . . . . . 36

$14 \quad$ F2-Overall results for crossover and mutation. . . . . . . . . . . 38

15 F6-Pooled ANOVA analysis. . . . . . . . . . . . . 38

$16 \quad$ F6-Overall results for crossover and mutation. . . . . . . . . . . . 41

17 ANOVA results of crossover $80 \%$ to $100 \%$ for FN5. . . . . . . 50

18 Relationship between Local Minima and Detrimental Crossover . . 85 
A-1 F1-Power with 100 replicates . . . . . . . . . . . . . 105

A-2 F1-Power with 100 replicates continued . . . . . . . . . 106

A-3 F1-Power with 500 replicates . . . . . . . . . . . . . . 107

A-4 F1-Power of the pooled analysis . . . . . . . . . . . . . 108

A-5 F3-Power of the pooled analysis . . . . . . . . . . . . . 109

A-6 $\quad$ F2-Power of the pooled analysis . . . . . . . . . . . . . . . . 110

A-7 F6-Power of the pooled analysis f . . . . . . . . . . . . 111

A-8 F6-Power of the pooled analysis for crossover $0 \%$ to $15 \%$. . . . . 112

A-9 F1-Partitioned sum of squares with 100 replicates . . . . . . . . . 113

A-10 F1-Partitioned sum of squares with 500 replicates . . . . . . . . 114

A-11 F1-Partitioned sum of squares of pooled analysis . . . . . . . . 115

A-12 F3-Partitioned sum of squares of pooled analysis . . . . . . . . . 116

A-13 F2-Partitioned sum of squares of pooled analysis . . . . . . . . . . 117

A-14 F2-Partitioned sum of squares of pooled analysis continued . . . . . 118

A-15 F6-Partitioned sum of squares of pooled analysis . . . . . . . . . . 119

A-16 F6-Partitioned sum of squares of pooled analysis continued . . . . . 120

A-17 F6-Partitioned sum of squares of pooled analysis continued . . . . . 121

A-18 F6-Partitioned sum of squares of pooled analysis for crossover . . . 122

A-19 Equations of fitted response curves . . . . . . . . . . . . . . . 123

B-1 ANOVA results of FN1 . . . . . . . . . . . . . . . . . 125

B-2 ANOVA results of FN2 and FN3 f . . . . . . . . . . 126

B-3 ANOVA results of FN4 and FN5 . . . . . . . . . . . . 127

B-4 ANOVA results of FN6 . . . . . . . . . . . . . . . . . 128

B-5 Equations of fitted response curves for FN1 to FN6 . . . . . . . . . 129 
B-6 Polynomial regression of FN1 to FN4 . . . . . . . . . . . . . . 130

B-7 Polynomial regression of FN5 and FN6 . . . . . . . . . . . . 131

C-1 ANOVA results of FN1R45 . . . . . . . . . . . . . . 133

C-2 ANOVA results of FN1R45 and FN2R45 . . . . . . . . . . 134

C-3 ANOVA results of FN4R45 and FN5R45 . . . . . . . . . 135

C-4 ANOVA results of FN6R45 and Landscape_20_101 . . . . . . . 136

C-5 Equations of fitted response curves for FN1R45 to FN6R45 . . . 137

C-6 Equations of fitted response curve for Landscape_20_101 . . . . . . 138

C-7 Polynomial Regression Tables for FN1R45 to FN6R45 and Landscape_20_101 . . . . . . . . . . . . . . . . . 139 


\section{List of Figures}

1 Dot diagram for F1. Each dot represents an instance of censoring. . 26

2a F1-Crossover response curve plot with 100 replicates. . . . . . . . 28

2b F1-Mutation response curve plot with 100 replicates. . . . . . . . . 29

3a F1-Linear curve fitted through simultaneous confidence intervals. . . 29

3b F1-Cubic curve fitted through simultaneous confidence intervals. . . 30

4a $\quad$ F1-Crossover response curve plot with 500 replicates. . . . . . . . 31

4b F1-Mutation response curve plot with 500 replicates. . . . . . . . 31

5a $\quad$ F1-Crossover response curve plot from pooled analysis. . . . . . . . 32

5b F1-Mutation response curve plot from pooled analysis. . . . . . . . 33

6a Fitted response curve: F1-crossover. . . . . . . . . . . . . . 33

6b Fitted response curve: F1-mutation. . . . . . . . . . . . . . . 34

7a Fitted response curve: F3-crossover. . . . . . . . . . . . . 35

7b Fitted response curve: F3-mutation. . . . . . . . . . . . 35

8a $\quad$ Fitted response curve: F2. . . . . . . . . . . . . . . . 37

8b Fitted response curve: F2-crossover. The solid line corresponds to the lower mutation rate of 0.18 and the top dotted line to the upper mutation rate of 0.24 . This applies to all subsequent figures. . . . . 37

8c Fitted response curve: F2-mutation. . . . . . . . . . . . . . . 38 
9a $\quad$ Fitted response curve: F6. . . . . . . . . . . . . . . . . 39

9b Fitted response curve: F6-crossover. . . . . . . . . . . . . . . . 40

9c Fitted response curve: F6-mutation. . . . . . . . . . . . . . . . 40

9d Fitted response curves for crossover 0\% and 10\%: F6-mutation. . . 41

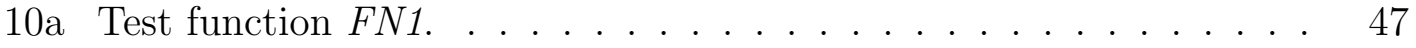

10b Test function $F N 6 . \quad \ldots \ldots \ldots \ldots$

11 Fitted response curves: FN5-crossover. . . . . . . . . . . . 50

12a Fitted response curve: FN5-overall. . . . . . . . . . . . . 51

12b Fitted response curve: FN6-overall. . . . . . . . . . . . 51

13a Fitted response curves FN5-mutation. . . . . . . . . . . 52

13b Fitted response curves FN6-mutation. . . . . . . . . . . . 52

14a Fitted response curve: FN3-overall. . . . . . . . . . . . . . 53

14b Fitted response curve: FN4-overall. . . . . . . . . . . . . 53

15a Test Function: FN3. . . . . . . . . . . . . . . . . . 59

15b Test Function: FN4. . . . . . . . . . . . . . . . . 60

16a Dot Diagram: FN3. . . . . . . . . . . . . 61

16b Dot Diagram: FN4. . . . . . . . . . . . . 61

17a Dot Diagram: $F N 3_{1 D} \ldots \ldots \ldots \ldots \ldots$

17b Dot Diagram: FN41D. . . . . . . . . . . . . . . 62

18a FN3 ${ }_{1 D}$ : Chromosome population after applying a low mutation rate. 63

18b FN $3_{1 D}$ : Chromosome population after selection. . . . . . . 63

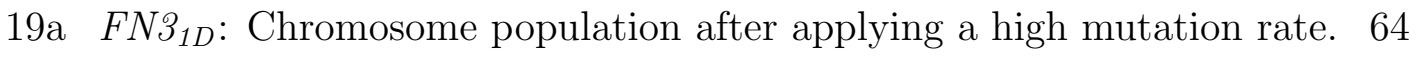

19b FN $3_{1 D}$ : Chromosome population after selection. . . . . . . . 64

20a FN41D: Chromosome population prior to applying mutation. . . . . 64 
20b FN41D: Chromosome population after applying a low mutation rate. 65

20c FN41D: Chromosome population after selection. . . . . . . . 65

21a $F N 3_{1 D}(\mathrm{HD}=$ Hamming Distance $) \ldots \ldots \ldots . \ldots 66$

$21 \mathrm{~b} F N_{41 D}(\mathrm{HD}=$ Hamming Distance $) \ldots \ldots \ldots \ldots 6$

22 Landscape 20_101 from the Huygens Suite. . . . . . . . . . . . 75

23a FN2R45 Initial Chromosome Population before Reproduction. . . . 79

23b FN2R45 Chromosome Population after Crossover. . . . . . . . . 80

23c FN2R45 Chromosome Population after Mutation. . . . . . . . 81

24 Mutation Plot for Test function FN2R45. . . . . . . . . . . . . 82

25 Probabilities associated with the movement of a single two bit chromosome after mutation. . . . . . . . . . . . . . . 82

26a Heat Map of FN2R45 illustrating location of local minima along X

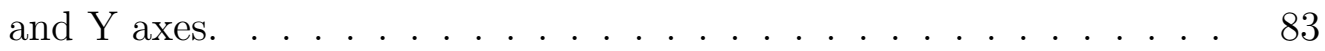

26b Heat Map of FN3R45 illustrating location of local minima along X

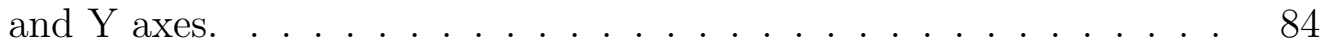

27a Response curve for test function FN2R45. . . . . . . . . . . 84

27b Response curve for test function FN3R45. . . . . . . . . . . . 86

28a Hamming Distances for FN2R45. . . . . . . . . . . . 86

28b Hamming Distances for FN3R45. . . . . . . . . . . . 87

29 Probability of jumping Hamming Gap versus Mutation rate. . . . . 88 


\section{Chapter 1}

\section{Introduction}

$\mathrm{S}$ ince the era of ENIAC, the first successful high speed-computer developed in the 1930s, an emerging component of computer science has been research into artificial intelligence (AI). This encompasses areas such as natural language processing, knowledge representation, automated reasoning, machine learning and evolutionary computation.

A practical application of AI has been the use of computers to solve problems. In order to formulate successful approaches researchers in artificial intelligence have looked to processes found in nature, such as evolution, for assistance. As such the development of this work has come under the heading of evolutionary computation, a general adaptable paradigm for problem solving especially well suited for optimization problems [2].

Such adaptive algorithms are search algorithms which can be used to find solutions to a variety of continuous and discrete problems. The general structure consists of a population of candidate solutions which are adapted in parallel during successive iterations with feedback obtained from an evaluation function [11]. Unlike algorithms that operate on a single solution, adaptive algorithms make improvements by combining the elements of good solutions to create better ones [34]. A classical example is genetic algorithms (GAs) [20]. While this thesis focusses on GAs 
it should be noted that the methodology is readily applicable to other adaptive algorithms.

\subsection{Genetic Algorithms}

GAs were originated by researchers including Holland who put forward the idea of developing adaptive algorithms based upon processes seen in genetics [20]. The relationship between genetics and GA terminology is illustrated in Table 1.

Table 1: Genetics and GA Terminology

\begin{tabular}{|c|c|}
\hline Genetic Terminology & $\begin{array}{c}\text { Realisation in GAs } \\
\text { GA Implementation }\end{array}$ \\
\hline \hline Chromosome & Bit-string \\
\hline Gene & Bit character \\
\hline Allele & Value 1 or 0 \\
\hline Locus & Bit-string position \\
\hline Genotype & Structure \\
\hline Phenotype & Decoded structure (solution) \\
\hline Epistasis & Nonlinearity \\
\hline
\end{tabular}

The classic GA works by encoding potential solutions to a problem as a series of bits or genes on a bit-string or chromosome. The mechanics of a GA are straightforward: in its simplest form new solutions are generated using crossover, where genes are swapped over between pairs of chromosomes, and mutation, where the binary value of a gene is inverted.

While the mechanics of a baseline GA are simple to describe and understand, the way in which a GA actually searches the solution space has been more complex to describe [2]. In addition, previously accepted aspects of GAs are being debated. For example, while it has been traditionally maintained that crossover is a necessary inclusion, the conjecture of naive evolution, where a GA contains selection and mutation only, places this in question $[12,39]$.

Such debates have been fuelled by the fact that little research has been done on how to decide whether a parameter significantly affects performance, how performance 
varies with respect to changes in parameters, whether there is any interaction between parameters, and what ultimately are the best values or range of values for the parameters which are implemented.

Given that there is no generally accepted methodology for exploring a GA in order to address these important basic issues the present thesis comprises the following:

1. The formulation of a rigorous methodology for the statistical exploratory analysis of GAs with its application to a number of benchmark problems;

2. The application of this methodology to the issue of the importance of the interaction between the crossover and mutation operators;

3. The application of this methodology to the issue of the relationship between the encoding that is used and GA performance;

4. The application of this methodology to the issue of the detrimentality of crossover for certain problems.

\subsection{Thesis Structure}

Expanding upon the above, the present thesis has the following structure:

Chapter 2 proposes a rigorous yet practical statistical methodology for the exploratory analysis of GAs. Section 2.1 of this chapter provides some background to the problem of analyzing GA performance. This is followed in Section 2.2 by a discussion of non-statistical exploratory work in this area. Section 2.3 examines work which has used a statistical construct, recognizing the appropriateness of statistical analysis to this problem. However, a number of limitations are found which include issues of experimental design, blocking, power calculations and response curve analysis. In Section 2.4 the newly formulated statistical methodology is described. Following this Section 2.5 illustrates the application of this methodology with case studies of benchmark problems from De Jong's [9] and Schaffer's 
[6] test suites. This includes some unexpected outcomes, particularly on the use of crossover. A discussion in Section 2.6 concludes this chapter.

Chapter 3 examines the issue of whether, in a GA, crossover and mutation interact or whether each exerts its effect independently. Section 3.1 discusses studies which have suggested that interaction between crossover and mutation may exist. Section 3.2 gives an overview of the way in which the statistical methodology presented in this thesis has been applied to a new test function, $F N n$, which has been utilized to demonstrate the existence of interaction between crossover and mutation. Section 3.3 links the existence of interaction between crossover and mutation with the difficulty of the function defined in terms of modality. Section 3.4 provides a concluding discussion to this chapter.

The first section of Chapter 4, Section 4.1, looks at the issue of the choice of encoding and its impact upon GA performance since GA practitioners report differing performances by changing the representation which is used $[6,37]$. Section 4.2 reviews the methods used to investigate this question, including a description of computer animation. Section 4.3 demonstrates how the choice of Gray encoding may have a statistically demonstrable effect upon the difficulty of a problem, utilizing results from both statistical analysis and computer animation. Section 4.4 provides a concluding discussion to this chapter.

Chapter 5 examines the issue of the detrimentality of crossover. This came about as a limited amount of data from the literature suggested that the niche for the beneficial effect of crossover upon GA performance may be smaller than has traditionally been held. Based upon not-linear-separable problems from earlier components of this thesis we decided to explore this by comparing two test problem suites, one comprising non-rotated functions and the other comprising the same functions rotated by 45 degrees rendering them not-linear-separable. Section 5.1 examines the issue of the detrimentality of crossover from the literature. Section 5.2 reviews work from the previous chapters of this thesis which prompted the present research. 
Section 5.3 briefly reviews the methods including any refinements to the statistical methodology. A discussion of the results obtained appears in Section 5.4 and Section 5.6. Section 5.5 examines factors affecting the detrimentality of crossover. Section 5.7 discusses the findings and suggests areas of future research.

Finally, Chapter 6 reviews general conclusions from this thesis. Limitations of the thesis are discussed and areas for future research are suggested. 


\section{Chapter 2}

\section{Statistical Methodology}

$\mathcal{1}_{\text {population of potential solutions [2]. The individuals interact either through }}$ the adaptation operators themselves, or through competitive selection mechanisms for determining subsequent generations. If the adaptation strategy is successful, the population (or part thereof) will converge on an optimal (or at least "good") solution. ${ }^{1}$

While the mechanics of each individual adaptation are quite straightforward, the way individual changes affect the success of the population as a whole is more difficult to determine. This is also true of the parameters that are used to fine tune, or improve the success of, adaptive algorithms. Examples include population size, mutation and crossover rates. Values for these parameters are most commonly set through a process of trial and error, or based on recommendations from related problems in the literature, rather than through statistically sound analysis of their affects on performance.

This chapter presents a methodology designed to assess the impact of these parameters on GA performance. The methodology addresses issues of experimental design, blocking, power calculation and response curve analysis. The approach is

\footnotetext{
${ }^{1}$ Readers unfamiliar with genetic algorithms are referred to [6] for a thorough introduction to GAs and examples of the range of applications to which they have been applied.
} 
demonstrated with case studies applying a baseline GA to benchmark problems from De Jong's [9] and Schaffer's [6] test suites.

\subsection{Background}

GAs are used in search and optimization problems, such as finding the maximum or minimum of a function in a given domain. The characteristics of GAs including bitstring encodings, randomization and operator without domain knowledge [1], have made the way in which a GA population converges on solutions has been more complex to describe [2]. Holland put forward the idea of schemata [20]: similarity templates describing a subset of strings with similarities at certain positions [17]. When the chromosome possesses these schemata its fitness improves. Operators such as crossover and mutation work by altering chromosomes to contain more good schemata. Goldberg elaborated by conceptualizing building blocks (highly-fit, shortdefining-length schemata) and implicit parallelism [17]. However, the increase in sophistication and differences in implementations of GAs, such as quantum-inspired GAs [31] and the use of transposition [40], has made it increasingly difficult to propose newer models of convergence.

In addition, previously accepted aspects of GAs are being debated. For example, while it has been traditionally maintained that crossover is a necessary inclusion, the conjecture of naive evolution, a GA which contains selection and mutation only, places this in question $[12,39]$. Such debates have been fuelled by the fact that little research has been done on how to decide whether a parameter significantly affects performance and how performance varies with respect to changes in parameters. There is currently no generally accepted methodology for exploring a GA in order to address these issues.

The difficulty in developing such a methodology is illustrated by problems encountered in both working from theoretical models and real world data. In the first 
instance, trying to formally describe GAs has been attempted using various mathematical approaches such as Markov chains [8, 19]. These approaches have been limited by the complexity of the calculations. Moreover, the assumptions made in much of the theoretical work may simply not be applicable nor attainable in practice. There has therefore been a realization that research involving real world data will be necessary in order to provide guidelines that may come to be generally accepted by GA practitioners.

Initial empirical work of this kind was carried out by De Jong [9] whose experiments resulted in a set of recommendations that came to represent early guidelines [39]. Later recommendations by Grefensette [18] using a meta-level GA (meta-GA) produced results which did not wholly agree with De Jong. The meta-GA approach is limited in that independent runs of the meta-GA can result in different best values. Furthermore, it does not provide any information as to whether any interaction occurs nor the trend of the performance behaviour over the range of values studied.

A limited number of studies have made use of statistical analysis, recognizing the ability of statistics to address many of these issues. However, as discussed in Section 2.3, these studies have been limited by failing to fully address important issues such as blocking for seed, calculating power and thorough response curve analysis. Thus, results and recommendations from these studies, though obtained from real practical experience, are still subject to debate.

The next sections look more closely at the various studies in this area. In doing so the inconsistency of the results and the limitations of the methodologies are noted.

\section{$2.2 \quad$ Non-Statistical Exploratory Analysis}

As stated above, there is currently no generally accepted methodology for analyzing the relationship between parameters and performance of a GA. Attempting to mathematically describe GAs is complex and has not resulted in practical guidelines. This has given rise to various empirical studies which attempt to provide such 
data. However, both the methodologies and results have varied.

Early work was provided by De Jong who altered the values of parameters such as population size, crossover rate and mutation rate in order to assess the effect on performance. This was defined in terms of online performance, the average performance of all chromosomes tested during the search, and offline performance, the current best chromosome value for each iteration [39]. Five test problems of increasing difficulty were used which became known as the De Jong suite [9]. Table 2 lists De Jong's recommendations for optimal performance for the parameters listed.

Table 2: Recommendations for basic parameter settings

\begin{tabular}{|c|c|c|}
\hline \multirow{4}{*}{ De Jong } & Population size & $50-100$ \\
\cline { 2 - 3 } & Crossover rate & 0.60 \\
\cline { 2 - 3 } & Mutation rate & 0.001 \\
\hline \hline \multirow{5}{*}{ Grefensette } & Population size & 30 (online) \\
\cline { 2 - 3 } & Population size & 80 (offline) \\
\cline { 2 - 3 } & Crossover rate & 0.95 (online) \\
\cline { 2 - 3 } & Crossover rate & 0.45 (offline) \\
\cline { 2 - 3 } & Mutation rate & 0.01 (online) \\
\cline { 2 - 3 } & Mutation rate & 0.01 (offline) \\
\hline \hline \multirow{5}{*}{ Freisleben and Härtfelder } & Population size & 100 (maximal) \\
\cline { 2 - 3 } & Crossover rate & 0.49 \\
\cline { 2 - 3 } & Mutation rate & 0.8 -0.93 \\
\hline
\end{tabular}

At this stage there was little evidence to dispel the idea that such data could serve as generic guidelines for different problem domains. Hence, these data came to represent guidelines for GA practitioners. Subsequent work, however, was not consistent with these recommendations.

This is illustrated in the results of Grefensette who pioneered the use of meta-GAs [18] for finding optimal values for parameters. His results for the De Jong suite are shown in Table 2. Other studies using the meta-GA approach also produced differing results, as seen in the work by Freisleben and Härtfelder [16] in the domain 
of neural network weights optimization (see Table 2).

\subsection{Statistical Exploratory Analysis}

As the previous studies did not clarify the relationship between parameters and performance statistical analysis has been used for this purpose. For example, Schaffer et al [39] conducted a factorial design study using the analysis of variance (ANOVA). This study used the De Jong suite plus an additional five problems. The recommendations for best online performance from this study are shown in Table 3. Close examination of the best online pools suggested a relative insensitivity to crossover which in turn suggested that naive evolution may be a powerful search algorithm in its own right when using bit-string encoding $[12,39]$. Work by Yao, Liu and Lin suggests that this may also be true when using real values [43]. These data challenge the traditional assumption that the crossover operator is a necessary inclusion in a GA [6].

Statistics was also used by Petrovski, Wilson and McCall [33] who carried out fractional factorial experiments in the domain of anti-cancer chemotherapy. These were combined with linear regression in order to pinpoint which parameters were significant and estimate their best values. The outcome measure, $\Psi$, was the number of generations required in order to reach the feasible region in the solution space. The results are shown in Table 3.

Table 3: Recommendations for basic parameter settings using statistics.

\begin{tabular}{|l|c|c|}
\hline \multirow{3}{*}{ Schaffer et al } & Population size & 20-30 (online) \\
\cline { 2 - 3 } & Crossover rate & $0.75-0.95$ (online) \\
\cline { 2 - 3 } & Mutation rate & $0.005-0.01$ (online) \\
\hline \hline \multirow{3}{*}{$\begin{array}{l}\text { Petrovski, Wilson } \\
\text { and McCall }\end{array}$} & Crossover rate using $\Psi$ & 0.6146 \\
\cline { 2 - 3 } & Mutation rate using $\Psi$ & 0.1981 \\
\cline { 2 - 3 } & Crossover rate using $\log (\Psi)$ & 0.7600 \\
\cline { 2 - 3 } & Mutation rate using $\log (\Psi)$ & 0.1069 \\
\hline
\end{tabular}


In overview, it is clear from both the non-statistical and statistical approaches that results have varied, notably for mutation where the more recent studies, including those using statistics, suggest higher rates. This may indicate a more complex effect for this parameter or alternatively that best values are problem specific. Moreover, the influence of differing problem domains must also be considered [42].

Importantly, however, the variation seen in these studies may also be a result of the differing methodologies that have been employed and therefore suggests the need to develop a generally accepted methodology for carrying out such exploratory work. While statistics is promising for this purpose, a number of limitations need to be addressed.

First, little attention has been given to blocking for seed as a source of variation or noise. As pointed out by Davis [7], finding good settings for parameters can be difficult due to the fact that the same parameter settings on the same problems can lead to different results. In practice these differences can be traced to different pseudo-random number generator seeds in the initialization of populations and in the implementation of selection, crossover and mutation. Blocking for seed by grouping experimental units into homogenous blocks, so that each run of the GA for differing levels of crossover and mutation occurs with the same seeds, limits the cause of variation within blocks to the parameters under study. In this way variation or noise is reduced and comparisons are sharpened [24].

Adding to this, issues dealing with the calculation of power and sample size have been ignored. This has meant that it is uncertain whether the studies carried out have had adequate power and thus sample size to detect differences that could be considered noteworthy. Sample sizes which are too small will generally fail to result in statistical significance. This is particularly important if blocking is not carried out since the data-set is akin to a completely randomized design. In such a design effects may not be detected due to the extent of background noise in the data-set produced by seed. Thus, a much larger sample size is required to detect effects of interest. 
A detailed analysis of response curves has also been limited. It is important to undertake such an analysis as it allows one to study the behaviour of the parameter over the range of values implemented. Such data are useful in the optimization process. For example, knowing that a parameter has a linear relationship to performance may suggest that either the value for the parameter is set as high as possible or that the parameter is excluded.

In the next section the experimental set-up is defined and the statistical methodology is described.

\subsection{Methods}

Before describing our methodology we briefly introduce the test functions and the GA used to illustrate our approach.

\subsubsection{Choice of Standard Test Functions}

It was important to select test functions which are well known. Initially, the first three problems from the De Jong [9] suite were tackled which are relatively easy for a GA to solve. This provided a useful set of problems, widely referenced in the literature, on which to demonstrate the initial applicability of the statistical methodology. These were F1 known as the SPHERE, F3 known as the STEP function and F2 known as ROSENBROCK'S SADDLE.

Next a more difficult problem, Schaffer's F6 [6], was tackled. These were all implemented as minimization problems and are displayed in Equation 1, Equation 2, Equation 3 and Equation 4, respectively:

$$
\begin{gathered}
f_{1}(\mathbf{x})=\sum_{i=1}^{3} x_{i}^{2},-5.12 \leq x_{i} \leq 5.12 \\
f_{3}(\mathbf{x})=\sum_{i=1}^{5}\left\lfloor x_{i}\right\rfloor,-5.12 \leq x_{i} \leq 5.12
\end{gathered}
$$




$$
\begin{gathered}
f_{2}(\mathbf{x})=100\left(x_{2}-x_{1}^{2}\right)^{2}+\left(1-x_{1}\right)^{2},-2.048 \leq x_{i} \leq 2.048 \\
f_{6}(\mathbf{x})=0.5+\frac{\left(\sin \sqrt{x_{1}^{2}+x_{2}^{2}}\right)^{2}-0.5}{\left(1.0+0.001\left(x_{1}^{2}+x_{2}^{2}\right)\right)^{2}},-100.0 \leq x_{i} \leq 100.0 .
\end{gathered}
$$

\subsubsection{Implementation of the GA}

The GA was implemented as detailed in Table 4. The implementation of the GA was deliberately simple so that a clear and concise demonstration of the proposed methodology and results could be made.

In this regard parameters such as the population size and bits per variable were not varied but kept at the values shown in Table 4 and only crossover and mutation were investigated in the present Thesis. The same methodology can be straightforwardly applied to the many other parameters suggested in the literature.

\subsubsection{Experimental Design and Statistical Test}

In order to decide upon the most appropriate type of experimental design and statistical test it was necessary to address several items:

1. Blocking for variation or noise due to seed.

2. Choice of an appropriate statistical test.

3. Statistical testing of individual parameters and their interactions.

4. Response curve analysis. This should allow for an estimate to be made of the best value for individual parameters with confidence intervals.

\footnotetext{
${ }^{2}$ Probabilistic selection used here is the random selection of parents with the probability of selection being directly proportional to the fitness of a chromosome.

${ }^{3}$ Mutation is implemented as described by Davis [6]. That is, if the probability test is passed the binary bit is replaced by another binary bit that is randomly generated. Approximately fifty per cent of the time the new bit will be the same as the old bit. The bit-flipping mutation rate is therefore half of the implemented mutation rate.
} 
Table 4: Details of the GA

\begin{tabular}{|l|l|}
\hline Variable representation & Bit-string \\
\hline Bits per variable & 22 \\
\hline Genes & Binary value 1 or 0 \\
\hline Population size & 50 chromosomes \\
\hline Chromosome coding & Gray coding \\
\hline Selection & Probabilistic selection ${ }^{2}$ \\
\hline Experimental unit & $\begin{array}{l}\text { Blocks containing independent runs } \\
\text { of the GA for different } \\
\text { crossover and mutation rates } \\
\text { with the same seeds }\end{array}$ \\
\hline Crossover & $\begin{array}{l}\text { Single point }(\text { randomly selected) } \\
\text { per variable }\end{array}$ \\
\hline Mutation & $\begin{array}{l}\text { Randomly generated bit replacement } \\
3\end{array}$ \\
\hline Performance measure & $\begin{array}{l}\text { Final epoch ie } \\
\text { epoch at which fitness of best } \\
\text { chromosome } \leq 10^{-10} \text { of maximum fitness } \\
\text { for F1, F2 and F3 } \\
\text { and } \\
\text { epoch at which fitness of best } \\
\text { chromosome } \leq 10^{-6} \text { of maximum fitness } \\
\text { for F6 }\end{array}$ \\
\hline
\end{tabular}

5. Calculation of power.

6. A methodology that is rigorous yet practical enough to be undertaken with common statistical packages and available desktop computing power.

7. Statistical principles that can be generically applied to other adaptive algorithms.

These are discussed in turn.

\section{Blocking.}


The variation seen in GA runs is due to the differences in the starting population and the probabilistic implementation of mutation and crossover. This is in turn directly dependent on seed: the value used to generate the pseudo-random sequences. In usual implementations of a GA the effect of seed is not regulated and so the experimental design may be conceived as being entirely randomized. In order to demonstrate statistically significant effects a very large data-set is required in order to detect effects over and above variation or noise due to seed.

To address this issue, it was necessary to control for the effect of seed via the implementation of a randomized complete block design. In such a design every combination of levels of parameters appears the same number of times in the same block and in the present study the blocks are defined through seeds. For example, if there are $i$ levels of parameter A and $j$ levels of parameter B then each block contains all ij combinations.

Seed is used for blocking, thus ensuring that the seeds used to implement items such as initialization of the starting population of chromosomes, selection, crossover and mutation are identical within each block. An increase in sample size occurs by replicating blocks identical except for the seeds. This is illustrated in Table 5. Replicates of this type are necessary to assess whether the effects of parameters are significantly different from variation due to other factors not controlled through seed.

Table 5: Creating a data-file from replicates of blocks.

\begin{tabular}{|c|c|c|c|}
\hline Block & Parameter A & Parameter B & Observations \\
\hline \hline Seed/s for block-replicate 1 & i levels & j levels & $i j$ \\
\hline Seed/s for block-replicate 2 & i levels & j levels & $i j$ \\
\hline Seed/s for block-replicate 3 & i levels & $j$ levels & $i j$ \\
\hline$\vdots$ & $\vdots$ & $\vdots$ & $\vdots$ \\
\hline Seed/s for block-replicate n & i levels & jlevels & $i j$ \\
\hline \hline \multicolumn{3}{|c|}{ Total observations $=i j n$ where $i j \geq 2$} \\
\hline
\end{tabular}




\section{ANOVA.}

In order to compare performances for 2 or more parameters using a randomized complete block design the statistical test for the equality of means known as the analysis of variance (ANOVA) was used. In ANOVA the null hypothesis is that the means for different levels of a parameter are equal. The alternative hypothesis is that the means for levels of a parameter are not equal and thus we conclude that the parameter has an effect upon the response variable. The effect of one parameter on this response variable may depend on the level of the other parameters. This is known as interaction. ANOVA also formally tests whether interaction is present or not.

ANOVA is so called as it essentially splits the total variation in the observations into variation contributed by the parameters (crossover and mutation), their interaction, block and error. Error is conceptualized in terms of residuals which are simply the individual deviations of the observations from the expected values.

Testing to ascertain if a parameter such as crossover or mutation has a statistically significant effect is a straightforward process. Firstly, the variation contributed by the parameter adjusted by the number of levels of the parameter is divided by the variation contributed by error adjusted by the number of levels of the parameters and the observations. This results in a ratio which is called an F value. Secondly, the probability that one would observe an F value as large as that which is calculated under the null hypothesis is determined. This is the p-value associated with the $\mathrm{F}$ value or simply $\operatorname{Pr}(\mathrm{F})$.

If the p-value is equal to or less than a chosen level of significance (see Section 2.4.4) this is taken to suggest that the parameter has an effect upon the response variable. A typical output from ANOVA is shown in Table 7 (see page 28). If we examine the p-values at the $1 \%$ level of statistical significance, we see that both crossover and mutation are highly significant. On the other hand, the interaction term, with a p-value of 0.61 , is non-significant. This 
means that there is no interaction occurring among crossover and mutation. In other words, crossover and mutation are acting independently of each other.

In ANOVA the values for $\operatorname{Pr}(\mathrm{F})$ (p-values) are only (exactly) valid if the responses are normally distributed. Although even moderate departures from normality do not necessarily imply a serious violation of the assumptions on which ANOVA is based [30], particularly for large sample sizes, it is standard procedure to use methods such as plotting a histogram of the residuals or constructing a normal probability plot of the residuals to verify normality of the sampling populations. In the present research, analysis of the residuals did not provide any evidence suggesting that the assumptions on which ANOVA calculations are made were compromised.

\section{Testing individual parameters and interaction.}

ANOVA allows for the testing of significance of individual parameters permitting the effect of crossover and mutation to be statistically demonstrated. For issues which have been raised in the literature such as naive evolution $[12,39]$, ANOVA provides evidence which may or may not support the inclusion of the crossover parameter.

In addition, ANOVA allows for the testing of interaction between parameters. Interaction is simply the failure of one parameter to produce the same effect on the response variable at different levels of another parameter [30]. Examining interaction is important because a significant interaction means the effect of each parameter cannot be considered independently of the others. The interaction parameter is created by multiplying the crossover parameter by the mutation parameter and adding this parameter to the ANOVA model.

\section{Response curve analysis.}

In ANOVA once a parameter is demonstrated to be statistically significant the effect of the parameter may be modelled through an appropriate 
polynomial. Statistical testing can be carried out to assess if the shape of the response curve is predominantly linear or is comprised of higher order polynomials by partitioning the total variation of each parameter into its orthogonal polynomial contrast terms.

Once the shape of the response curve is established, polynomial regression can be carried out to obtain estimates of the coefficients of the various parameters in the response curve equation. Importantly, if the interaction parameter is significant in the ANOVA model then the overall equation must be found. If not, then the equations for crossover and mutation can be obtained separately.

For fitted response curves which are comprised of quadratic or higher components we can obtain the derivatives and find the values where the derivatives equal zero which yield estimates of the best value for each parameter. Additionally, confidence intervals can be calculated if of interest.

However, if the fitted response curve is linear then a negative coefficient will correspond solely to a best rate of $100 \%$ while a positive coefficient will correspond solely to a best rate of $0 \%$ since the minimum of a straight line can only occur at either end.

5. Power.

The calculation of power for ANOVA can be made by using the effect size index, $f$, as described by Cohen [5]. Power is discussed in detail in Section 2.4.6.

6. Availability.

ANOVA and regression are standard statistical models available in virtually all statistical software packages which are used on desktop computers.

\section{Applicability.}

Randomized complete block design can be applied to other adaptive algorithms with little difficulty. It simply requires that the seeds, or any other 
sources of noise, are kept identical within each replicate so that the source can be blocked.

The GA was implemented in Java [41]. Statistical analysis was carried out using S-PLUS [21]. Power calculations were carried out using GPOWER [14].

A number of aspects of the analysis are discussed in more detail below.

\subsubsection{Choice of Level of Significance}

There are 2 types of errors associated with statistical testing. A type I error is the rejection of the null hypothesis when it is true. A type II error is the non-rejection of the null hypothesis when the alternative hypothesis is true. The probability of making a type I error is denoted by $\alpha$ and the probability of a type II error is denoted by $\beta$. Since the null hypothesis represents the most conservative proposal it is considered that a type I error is more serious than a type II error [24]. Thus, $\alpha$ is generally and arbitrarily set at a low level. This level of significance is traditionally set at values such as $10 \%, 5 \%$ or $1 \%$.

For published research a level of significance of $1 \%$ is often used [26]. P-values less than $1 \%$ suggest that the null hypothesis is strongly rejected or that the result is highly statistically significant [24]. In the present study we have employed $1 \%$ as our level of significance and correspondingly calculated $99 \%$ confidence intervals.

\subsubsection{Level of Significance for Orthogonal Simultaneous Mul- tiple Comparisons}

In a situation of orthogonal simultaneous multiple comparisons within a parameter it is necessary to modify the level of significance. This is because the probability of achieving one or more statistically significant results in $n$ simultaneous multiple comparisons will exceed the level of significance chosen ( $1 \%$ in the present study). 
This is illustrated in Equation 5.

$$
P(\text { at least one significant result in } n \text { independent tests })=1-(1-\alpha)^{n} \text {. }
$$

This occurs in ANOVA when the sum of squares for each parameter is partitioned into orthogonal contrast terms. In order to ensure that the probability of achieving one or more statistically significant results in $n$ simultaneous multiple comparisons is exactly 1\%, a modified level of significance was used for testing each of $n$ orthogonal polynomial contrast terms calculated in accordance with Equation 6.

$$
\text { Modified level of significance }=1-(1-\alpha)^{\frac{1}{n}} \text {. }
$$

Our approach is different from the Bonferroni method [21] which would simply divide the overall level of significance by the number of simultaneous multiple comparisons. The Bonferroni method will ensure that the probability of achieving one or more statistically significant results in $n$ simultaneous multiple comparisons is no greater than 1\%. Thus, it yields an upper bound such that the actual probability of achieving one or more statistically significant results in $n$ simultaneous multiple comparisons may be much smaller.

\subsubsection{Power}

As $1-\beta$ is the probability of rejecting the null hypothesis when it is false, this is known as the power of the test. A power of $80 \%(\beta=0.2)$ when there is moderate departure from the null hypothesis is considered desirable by convention [5]. The value of $\beta$ is related to sample size. A sample size that is too small will generally fail to produce a significant result while a sample size that is too large may be difficult to analyze (due to difficulties of handling large data sets) and wastes resources. It is therefore necessary to have some means of calculating whether the size of the sample chosen has sufficient power.

In order to calculate power it is necessary to specify the degree to which the null hypothesis is false. This is quantifiable as a specific non-zero value using the unit-less 
effect size indices $d$ and $f$ as described by Cohen [5]. For ANOVA, by convention, a small effect size is an $f$ value of 0.10 , a medium effect size is an $f$ value of 0.25 and a large effect size is an $f$ value of 0.40 .

In this part of the present study differences in a specified number of epochs were first converted to the effect size index, $d$, where:

$$
d=\frac{\mu_{\max }-\mu_{\min }}{\sigma}
$$

where $\mu_{\max }$ is the maximum mean over the levels of this parameter, $\mu_{\min }$ is the smallest population mean over the levels of this parameter, and $\sigma$ is the population standard deviation.

This results in a unit-less number to index the degree of departure from the null hypothesis of the alternative hypothesis, or more simply, the effect size one wishes to detect [5].

Next, the conversion from $d$ to $f$ for ANOVA requires a knowledge of the pattern of separation for all means for all $k$ levels of the parameter. Patterns identified by Cohen [5] are: 
1. Minimum variability: one mean at each end of $d$, the remaining $k-2$ means all at the midpoint.

2. Intermediate variability: the $k$ means equally spaced over $d$.

3. Maximum variability: the means are all at the end points of $d$.

Tables are available for the conversion from $d$ to $f$ for each scenario. If the pattern of separation is unknown an inspection of these tables illustrates that the most conservative approach is to assume the minimum variability pattern which results in $f$ being at its smallest. In this case $f$ is calculated as:

$$
f=d \sqrt{\frac{1}{2 k}} .
$$

It should be noted that power may be calculated a priori or post hoc. If the population standard deviation is known from prior research one can calculate $a$ priori the sample size required to confer a specified power. On the other hand, if the population standard deviation is unknown but can be estimated once the study is concluded then post hoc power calculations indicate the ability of the present sample size to detect specified effect sizes, given by Equation 7 .

As the present thesis was exploratory in nature and a priori assumptions about the population standard deviation could not be made post hoc calculations were strictly adhered to. Thus, while statistical significance had not been demonstrated in the ANOVA analysis for the interaction parameter, we continued to increase sample size by a factor of 5 . This was enacted until at least $80 \%$ power was achieved for detecting a difference of 5 epochs for the interaction between crossover and mutation. This is because $f$ is smallest for the interaction parameter since $k$ is greatest for this parameter.

As a final remark, in the present research the calculation of power was based upon the ability to detect a difference of at least 5 epochs as noted above. This number was chosen as it most closely approximated the difference in the number of epochs 
detectable for the simplest problem, $F 1$, if one had calculated power using an $f$ of 0.4 (large effect).

\subsubsection{Simultaneous Confidence Intervals for the Plotted Re- sponse Curve}

Plotting mean performance against parameter levels provides an initial estimate of the shape of the response curve. However, the shape of the curve may be compromised if the sample size is insufficient. To gauge the reliability of the trend $99 \%$ simultaneous confidence intervals about each mean can be calculated. The $z$ value for calculating simultaneous confidence intervals for $n$ levels of an individual parameter corresponds to the probability given by equation 9 .

$$
\mathrm{P}_{Z \text { value }}=1-\left(\frac{1-0.99^{\frac{1}{n}}}{2}\right) .
$$

Note that while confidence intervals tighten as sample size increases, showing increased confidence about the location of the population mean, there is still a great deal of randomness in each individual run.

\subsubsection{Pooled Analysis Design}

If large data-sets are required these may not be able to be analyzed when a parameter has too many levels, as this results in the statistical software having to deal with too many and too large matrices. In order to address this issue we devised a pooled analysis design for the present study as follows:

1. For each individual experiment we calculated the mean of the performance measure for each combination of crossover and mutation.

2. These data from individual experiments were concatenated into a new pooled data file. The response variable was now the mean of the performance measure averaged over the number of replicates in the individual experiment. This 
results in a smaller error variance, as the average of a number of observations is expected to be closer than a single observation to the population mean.

Each individual experiment denoted one level of the block parameter.

3. Analysis was carried out in the same manner as for individual experiments.

\subsubsection{Estimates of Best Values for Parameters}

Once the coefficients are obtained from the polynomial regression model it is straightforward to obtain an estimate of the best value for the specified parameter by differentiating and solving the response curve equation. 99\% confidence intervals are then calculated using Taylor's Expansion ( $\delta$ method) [36].

\subsubsection{Workup Procedures to Ensure a Balanced ANOVA Design}

A balanced design for ANOVA occurs if no data are missing or censored. In our case data is censored if that threshold is not reached and therefore stopping criterion not satisfied for a run of the GA. A balanced design is desirable since it results in the test statistic being more robust to small departures from the assumption of equal variances for the number of treatments. In addition, the power of the ANOVA test is maximized. This was achieved by two consecutive workup procedures which were carried out for all four test functions.

\section{Dot Diagrams}

First, to minimize the occurrence of censoring in the present study a crude exploration of the parameter space was conducted. A data-set of an arbitrary 10 replicates was generated for all functions using an interval of 0 to 1 for both the crossover (using an interval of 0.1) and mutation (using an interval of 0.01) parameters. If on at least one occasion the threshold was not reached for a particular 
crossover rate and mutation rate combination, this was shown as a dot on the resultant dot diagram.

Figure 1: Dot diagram for F1. Each dot represents an instance of censoring.

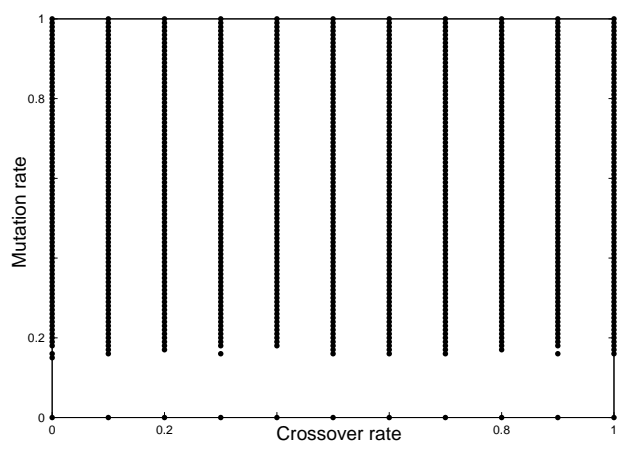

As illustrated in Figure 1, for $F 1$ mutation rates of less than 0.15 and greater than zero were not associated with censoring. In contrast, all crossover rates from 0 to 1 were valid. Thus, at this point for $F 1$ the rates which could be considered to be reasonably free from censoring, so that the threshold value would be reached or exceeded on every run of the GA, were crossover rates of 0 to 1 , and mutation rates of 0.01 to 0.14 . The dot diagrams were also found useful to give us an initial pictorial overview of the difficulty of a function (see Chapter 4).

\section{Finalizing ranges for exploratory statistical analysis}

Second, to further ensure that no censored data would appear in the data-sets for analysis, and so finalize the ranges for exploratory statistical analysis to begin, we conducted the following exercise.

Using crossover and mutation rates not associated with censoring from the dot diagrams, an arbitrary 10 data-sets of 100 replicates each were generated. Using S-PLUS the combination of crossover rate and mutation rate resulting in the best performance was found in each data-set. When these 10 combinations were collated they demonstrated the lowest and highest rates of crossover and mutation associated with best performance. For $F 1$ crossover ranged from 0.8 to 1 and mutation ranged 
from 0.05 to 0.08 .

However, to ensure that the ranges we would study could be considered robust we allowed the ranges to widen one interval step on either side. Thus, as displayed in Table 6 , this made the finalized range for $F 1$ for crossover 0.7 to 1 and for mutation 0.04 to 0.09 .

As a result of these two consecutive workup procedures, a balanced ANOVA design was achieved.

Table 6: Final ranges for crossover and mutation.

\begin{tabular}{|c|c|c|}
\hline Test function & Crossover final range & Mutation final range \\
\hline \hline F1 & $0.7-1$ & $0.04-0.09$ \\
\hline F3 & $0.8-1$ & $0.03-0.07$ \\
\hline F2 & $0-0.7$ & $0.18-0.24$ \\
\hline F6 & $0-0.7$ & $0.11-0.18$ \\
\hline
\end{tabular}

\section{$2.5 \quad$ Results}

\subsubsection{Exploratory Analysis of Test Function F1}

The results of analyzes of data-sets containing 100 replicates, 500 replicates and pooled results from 5 data-sets of 500 replicates are described consecutively to illustrate how statistics can be used to assist in exploratory analysis.

\section{Results with 100 Replicates}

Table 7 displays ANOVA of 100 replicates.

Crossover and mutation were both highly statistically significant while the interaction between crossover and mutation was not. Post hoc power calculations as shown in Table A-1 show that while the power for detecting a difference of 5 epochs 
Table 7: F1-ANOVA of 100 replicates.

\begin{tabular}{|c|c|c|c|c|c|}
\hline Parameter & Df & Sum of Sq & Mean Sq & F Value & $\operatorname{Pr}(\mathrm{F})$ \\
\hline \hline Crossover & 6 & 12347 & 2057.826 & 8.47756 & 0.0000000 \\
\hline Mutation & 10 & 58701 & 5870.091 & 24.18282 & 0.0000000 \\
\hline Interaction & 60 & 13664 & 227.733 & 0.93818 & 0.6117951 \\
\hline Block & 99 & 51956 & 524.813 & 2.16205 & 0.0000000 \\
\hline Residuals & 7524 & 1826361 & 242.738 & - & - \\
\hline
\end{tabular}

Residual standard error: 15.58005, Estimated effects are balanced.

was greater than $97 \%$ for both crossover and mutation the power for the interaction parameter was only $3.38 \%$. Thus, the use of 100 replicates was too small to demonstrate statistical significance for interaction.

The response curve plots for crossover and mutation are displayed in Figure 2a and Figure 2b. While the response curve plot for mutation suggested a quadratic trend, the response curve plot for crossover was less obvious. Since only 100 replicates were used the width of the simultaneous confidence intervals was very wide so that for crossover either a linear curve or a higher order polynomial such as a cubic curve could conceivably have fitted between the simultaneous confidence intervals.

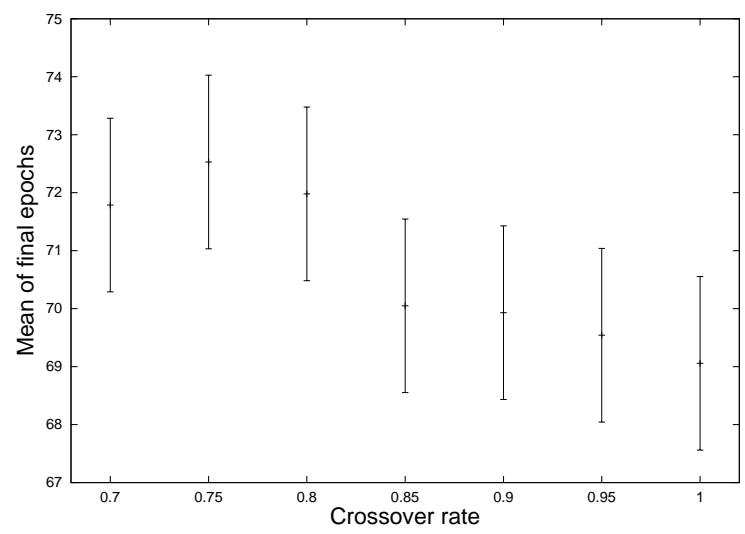

Figure 2a: F1-Crossover response curve plot with 100 replicates.

This is illustrated in Figure $3 \mathrm{a}$ and Figure $3 \mathrm{~b}$. As it is preferable to formally test for the shape of the response curve rather than relying on visual inspection, better information was obtained from the sum of squares partitioned into terms 


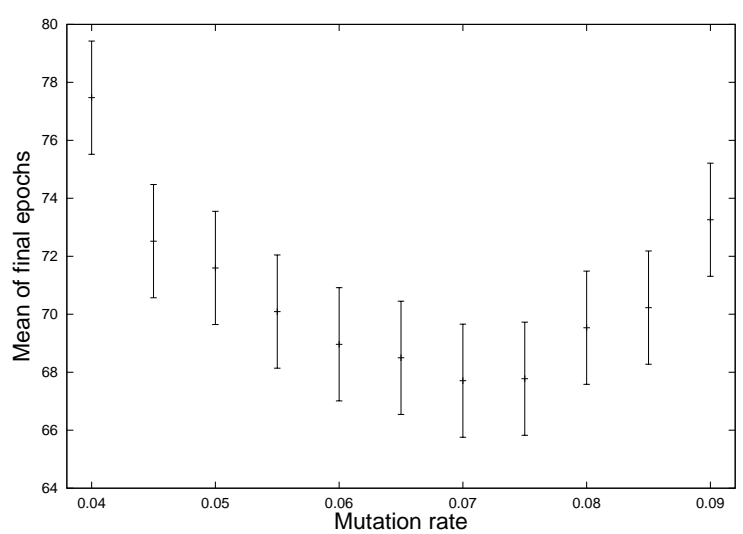

Figure 2b: F1-Mutation response curve plot with 100 replicates.

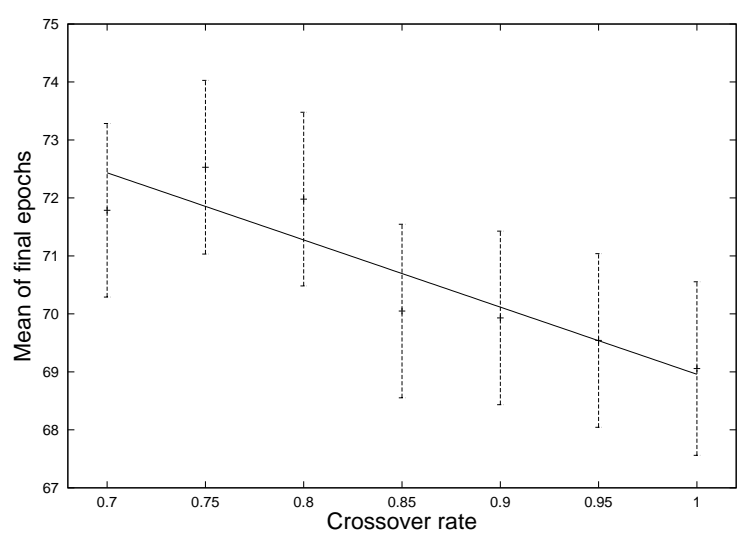

Figure 3a: F1-Linear curve fitted through simultaneous confidence intervals.

corresponding to orthogonal contrasts which represent polynomials. These data are shown in Table A-9 and suggested a linear trend for crossover and a quadratic trend for mutation.

However, given the lack of power associated with interaction it was necessary to repeat the analysis using an increased sample size. Adhering to our protocol of carrying out power calculations on a strictly post hoc basis we enacted a five fold increase in the number of replicates.

\section{Results with 500 Replicates}

ANOVA of 500 replicates is shown in Table 8. 


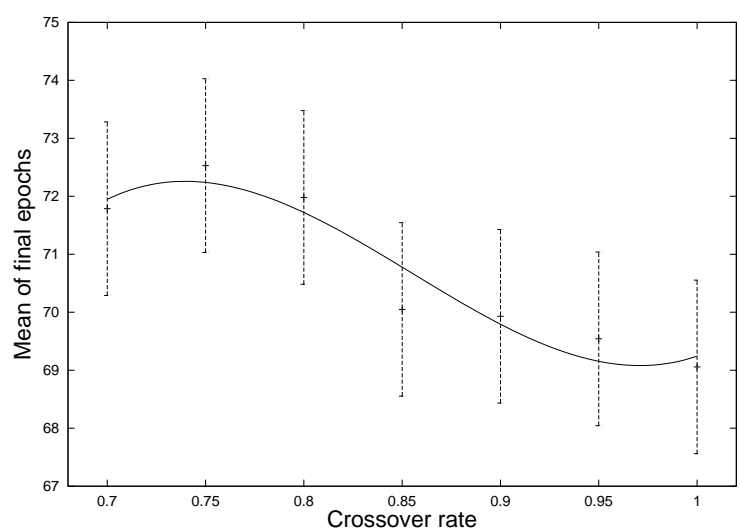

Figure 3b: F1-Cubic curve fitted through simultaneous confidence intervals.

Table 8: F1-ANOVA of 500 replicates.

\begin{tabular}{|c|c|c|c|c|c|}
\hline Parameter & Df & Sum of Sq & Mean Sq & F Value & $\operatorname{Pr}(\mathrm{F})$ \\
\hline \hline Crossover & 6 & 82952 & 13825.38 & 56.20533 & 0.0000000 \\
\hline Mutation & 10 & 208227 & 20822.75 & 84.65223 & 0.0000000 \\
\hline Interaction & 60 & 12386 & 206.44 & 0.83925 & 0.8079445 \\
\hline Block & 499 & 237465 & 475.88 & 1.93464 & 0.0000000 \\
\hline Residuals & 37924 & 9328542 & 245.98 & - & - \\
\hline
\end{tabular}

Residual standard error: 15.68375, Estimated effects are balanced.

A similar pattern for the overall results was evident. That is, a highly significant result for crossover and mutation while a non-significant result for the interaction parameter.

Table A-3 illustrates the improvement in power obtained by increasing the sample size though the power associated with the interaction parameter remained below the study threshold. The effect of increasing the number of replicates upon the width of the simultaneous confidence intervals for the response curves is shown in Figure $4 \mathrm{a}$ and Figure $4 \mathrm{~b}$. The increase in the number of replicates reduced the width of the simultaneous confidence intervals producing clearer linear behaviour for crossover and quadratic behaviour for mutation. Both trends were affirmed in the partitioned sum of squares displayed in Table A-10.

However, the continued lack of power associated with the interaction parameter 


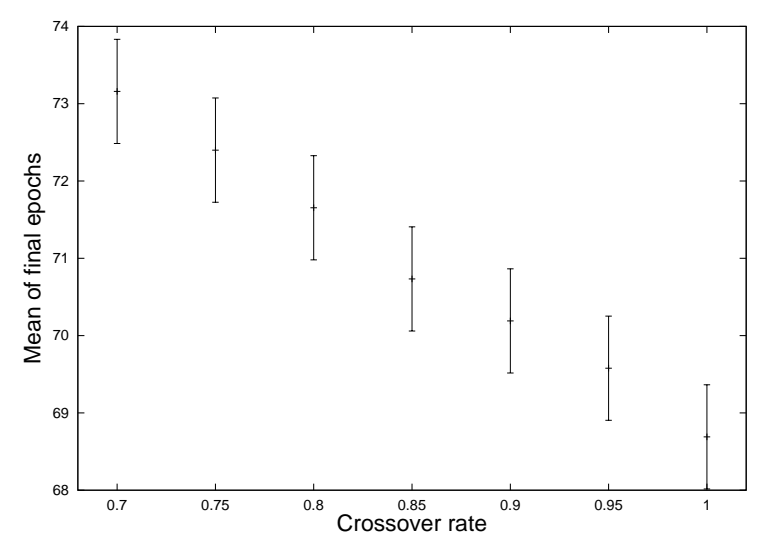

Figure 4a: F1-Crossover response curve plot with 500 replicates.

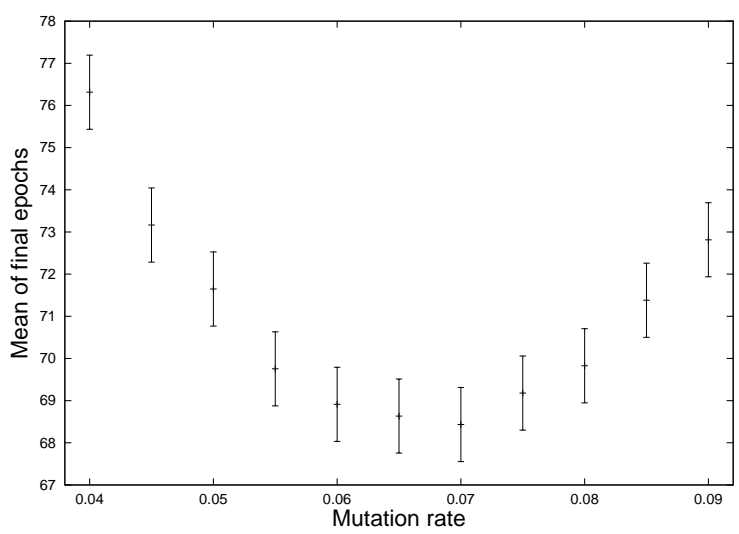

Figure 4b: F1-Mutation response curve plot with 500 replicates.

meant that a further increase in the sample size was again required. We opted again for a five fold increase in the number of replicates to 2500. However, this data-set could not be analyzed by S-PLUS due to the fact that the large number of levels for the block variable meant that the calculations involved too many and too large matrices. As such, the pooled analysis design was implemented.

\section{Results of the Pooled Analysis}

Table 9 shows ANOVA of the pooled data-set from 5 data-sets of 500 replicates. Both crossover and mutation were again highly statistically significant. However, the interaction between crossover and mutation was not with a p-value of 0.0377 . Post hoc power calculations are displayed in Table A-4. The increase in replicates 
Table 9: F1-Pooled ANOVA analysis.

\begin{tabular}{|c|c|c|c|c|c|}
\hline Parameter & Df & Sum of Sq & Mean Sq & F Value & $\operatorname{Pr}(\mathrm{F})$ \\
\hline \hline Crossover & 6 & 714.601 & 119.1002 & 256.1305 & 0.0000000 \\
\hline Mutation & 10 & 2153.876 & 215.3876 & 463.2010 & 0.0000000 \\
\hline Interaction & 60 & 38.977 & 0.6496 & 1.3970 & 0.0377493 \\
\hline Block & 4 & 1.381 & 0.3453 & 0.7426 & 0.5635587 \\
\hline Residuals & 304 & 141.359 & 0.4650 & - & - \\
\hline
\end{tabular}

Residual standard error: 0.6819076, Estimated effects are balanced.

now resulted in $100 \%$ power to detect a difference of 5 epochs for the interaction parameter. As the power threshold of the study had been exceeded it was not necessary to increase the sample size any further.

The response curve plots for crossover and mutation from the pooled analysis are displayed in Figure 5a and Figure 5b. As can be seen the width of the simultaneous confidence intervals has been further tightened. The partitioned sum of squares shown in Table A-11 illustrated strong agreement with the plots. However, for mutation a cubic effect was now significant though the quadratic effect remained predominant as evidenced when comparing the magnitude of the respective sum of squares.

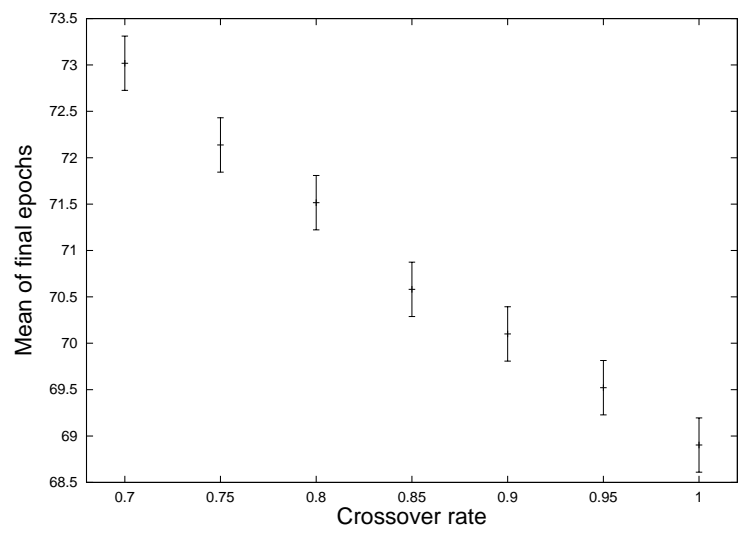

Figure 5a: F1-Crossover response curve plot from pooled analysis. 


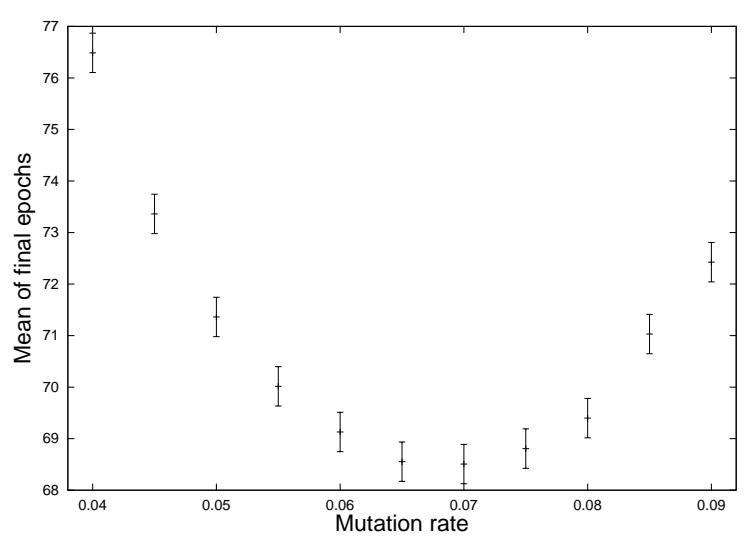

Figure 5b: F1-Mutation response curve plot from pooled analysis.

In conclusion, these data suggested that both crossover and mutation are highly important parameters in the GA for the $F 1$ problem domain. The behaviour of crossover is linear while the behaviour of mutation is predominantly quadratic with some cubic component. The interaction observed between crossover and mutation is not significant and therefore is of little practical importance.

Using polynomial regression separate fitted response curves for crossover and mutation were obtained. These are illustrated in Figure 6a and Figure $6 \mathrm{~b}$ and the equations are given in Table A-19. Using these equations the best values for crossover and mutation were calculated and the overall results are displayed in Table 10.

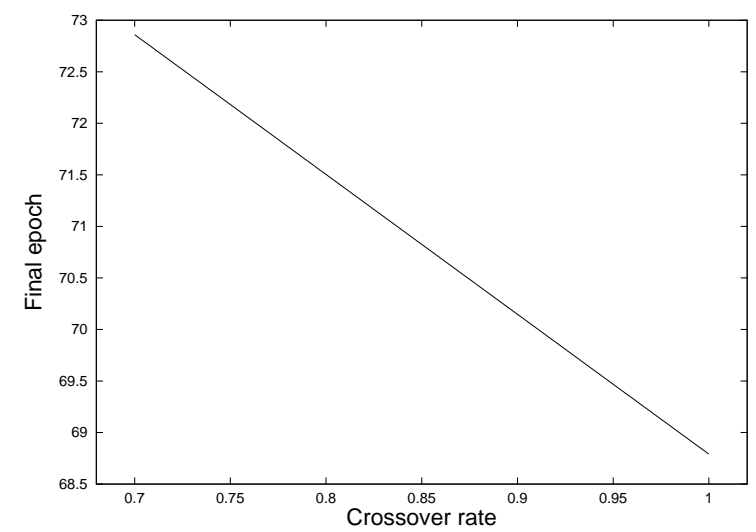

Figure 6a: Fitted response curve: F1-crossover. 


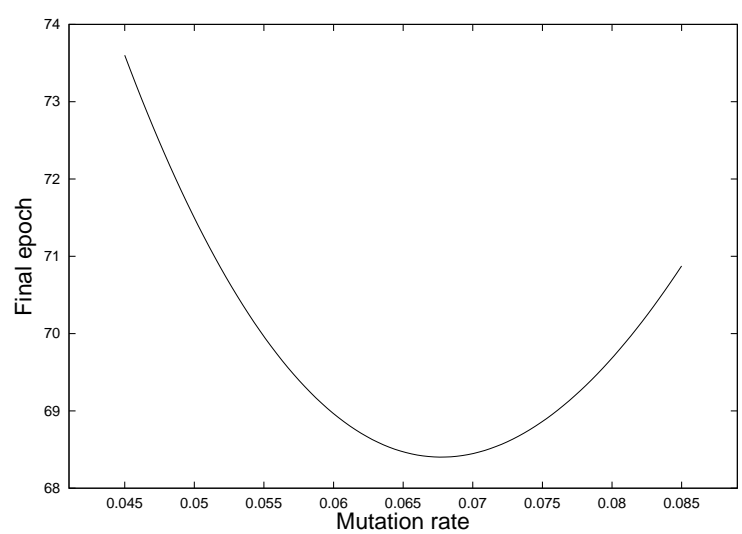

Figure 6b: Fitted response curve: F1-mutation.

Table 10: F1-Overall results for crossover and mutation.

\begin{tabular}{|c|c|c|c|}
\hline Parameter & Response curve shape & Estimated best value & $99 \%$ CI \\
\hline \hline Crossover & Linear & $100 \%$ & - \\
\hline Mutation & Cubic & $6.77 \%$ & $6.60 \%-6.95 \%$ \\
\hline
\end{tabular}

\subsubsection{Exploratory Analysis of Test Function F3}

Table 11: F3-Pooled ANOVA analysis.

\begin{tabular}{|c|c|c|c|c|c|}
\hline Parameter & Df & Sum of Sq & Mean Sq & F Value & $\operatorname{Pr}(\mathrm{F})$ \\
\hline \hline Crossover & 4 & 251.835 & 62.9588 & 51.8074 & 0.0000000 \\
\hline Mutation & 8 & 3460.606 & 432.5757 & 355.9567 & 0.0000000 \\
\hline Interaction & 32 & 50.045 & 1.5639 & 1.2869 & 0.1550913 \\
\hline Block & 4 & 12.390 & 3.0974 & 2.5488 & 0.0409906 \\
\hline Residuals & 176 & 213.884 & 1.2152 & - & - \\
\hline
\end{tabular}

Residual standard error: 1.102383, Estimated effects are balanced.

ANOVA of the pooled data-set for F3 is shown in Table 11. Crossover and mutation were highly statistically significant while the interaction between crossover and mutation was not. Post hoc power calculations displayed in Table A-5 show that the power for detecting a difference of 5 epochs for the interaction parameter was $88.27 \%$, exceeding the threshold for the present study. As such there was no further need to increase the sample size. 
An examination of the partitioned sum of squares shown in Table A-12 confirmed a linear trend for crossover and a quadratic trend for mutation. Using polynomial regression the fitted response curves for crossover and mutation were obtained. These are illustrated in Figure $7 \mathrm{a}$ and Figure $7 \mathrm{~b}$ and the equations given in Table A19. Using these equations the best values for crossover and mutation were calculated and the overall results are displayed in Table 12.

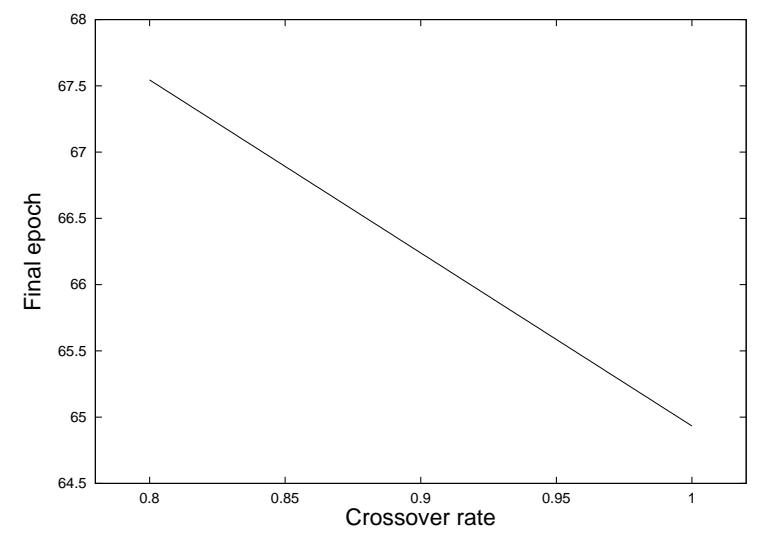

Figure 7a: Fitted response curve: F3-crossover.

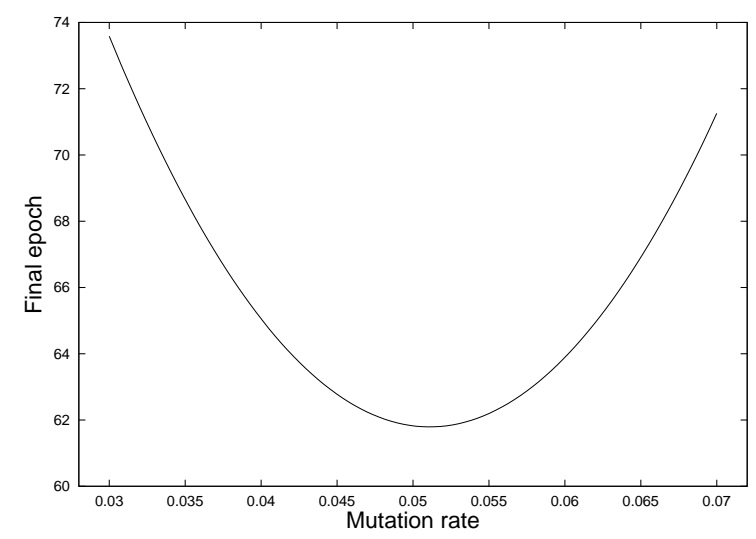

Figure 7b: Fitted response curve: F3-mutation.

Table 12: F3-Overall results for crossover and mutation.

\begin{tabular}{|c|c|c|c|}
\hline Parameter & Response curve shape & Estimated best value & $99 \%$ CI \\
\hline \hline Crossover & Linear & $100 \%$ & - \\
\hline Mutation & Quadratic & $5.11 \%$ & $5.07 \%-5.15 \%$ \\
\hline
\end{tabular}




\subsubsection{Exploratory Analysis of Test Function F2}

\section{Results of the pooled analysis}

Table 13 shows ANOVA analysis of the pooled data-set for F2.

Table 13: F2-Pooled ANOVA analysis.

\begin{tabular}{|c|c|c|c|c|c|}
\hline Parameter & Df & Sum of Sq & Mean Sq & F Value & $\operatorname{Pr}(\mathrm{F})$ \\
\hline \hline Crossover & 14 & 29291.3 & 2092.235 & 46.1088 & 0.000000000 \\
\hline Mutation & 12 & 103575.8 & 8631.317 & 190.2173 & 0.000000000 \\
\hline Interaction & 168 & 10717.5 & 63.795 & 1.4059 & 0.001550061 \\
\hline Block & 4 & 820.0 & 205.006 & 4.5179 & 0.001298162 \\
\hline Residuals & 776 & 35211.8 & 45.376 & - & - \\
\hline
\end{tabular}

Residual standard error: 6.736177, Estimated effects are balanced.

Crossover and mutation were highly statistically significant as was the interaction between crossover and mutation with a p-value of 0.00155. Since the interaction parameter demonstrated strong statistical significance no further increments in sample size were necessary.

Examination of the sum of squares partitioned into orthogonal polynomial contrast terms as shown in Table A-13 suggested a linear trend for crossover and a cubic trend for mutation with the predominant effect for the latter arising from the quadratic term. Partitioning of the sum of squares of the interaction parameter showed only a statistically significant effect ( $p$-value less than 0.01 ) for the linear:linear term (that is, the linear component of crossover multiplied by the linear component of mutation).

As the interaction parameter was found to be significant, in contrast to the results for F1 and F3, polynomial regression incorporating the linear by linear interaction effect was used to obtain the overall 3-dimensional equation for the response curve and this is given in Table A-19. Figure 8a illustrates this overall 3-dimensional response curve and Figure 8b and Figure 8c illustrate 2-dimensional slices corresponding to crossover and mutation, respectively. 


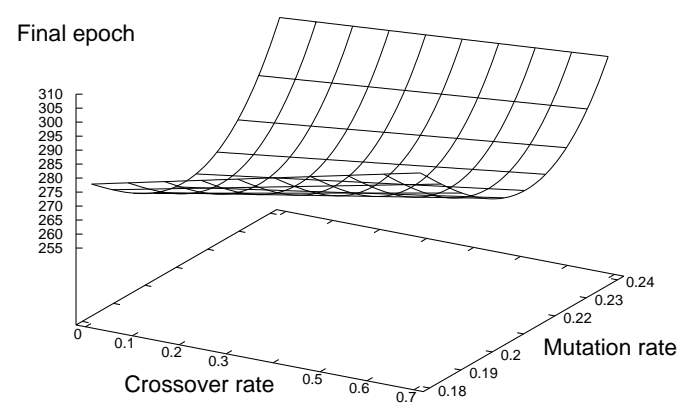

Figure 8a: Fitted response curve: F2.

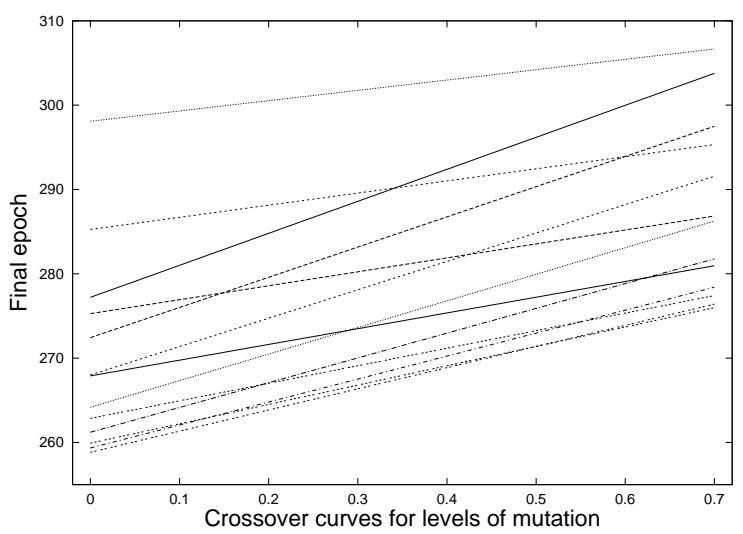

Figure 8b: Fitted response curve: F2-crossover. The solid line corresponds to the lower mutation rate of 0.18 and the top dotted line to the upper mutation rate of 0.24. This applies to all subsequent figures.

Figure 8b illustrates consistent positive slopes for the crossover curves indicating a worsening of performance as the crossover rate increased. Additionally, it should be noted that the top curve (the solid curve) and the second curve from the top correspond to mutation values of $24 \%$ and $18 \%$, respectively. As the other curves fall inside these extremes this illustrates how this cross-section actually curves into the page. In Figure 8c we see the curved trend of each mutation curve. In this graph, the top curve corresponds to a crossover rate of $70 \%$ and the bottom curve corresponds to a crossover rate of $0 \%$. This suggests that mutation performs best when the crossover rate is $0 \%$.

Using the equation where the rate of crossover was $0 \%$ the best value for mutation 


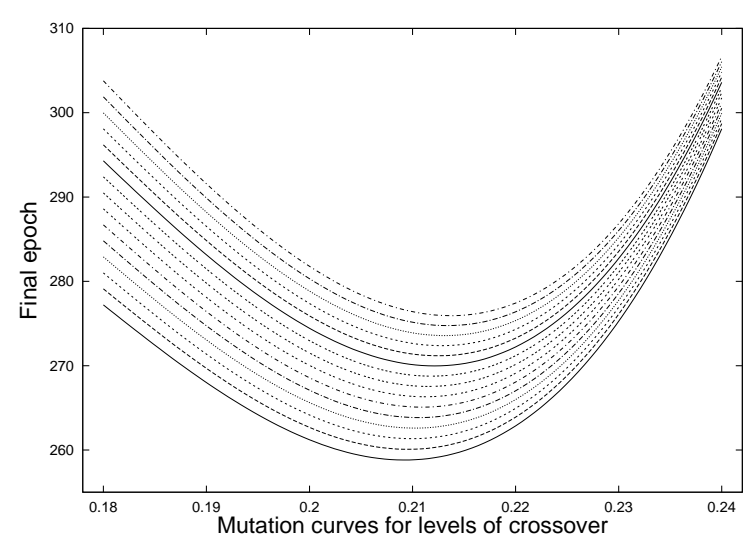

Figure 8c: Fitted response curve: F2-mutation.

was calculated. The overall results of the analysis are shown in Table 14.

Table 14: F2-Overall results for crossover and mutation.

\begin{tabular}{|c|c|c|c|}
\hline Parameter & Response curve shape & Estimated best value & $99 \%$ CI \\
\hline \hline Crossover & Linear & $0 \%$ & - \\
\hline Mutation & Cubic & $21.15 \%$ & $21.01 \%-21.30 \%$ \\
\hline Interaction & Linear:Linear & - & - \\
\hline
\end{tabular}

\subsubsection{Exploratory Analysis of Test Function F6}

\section{Results of the pooled analysis}

Table 15 shows ANOVA analysis of the pooled data-set for $F 6$.

Table 15: F6-Pooled ANOVA analysis.

\begin{tabular}{|c|c|c|c|c|c|}
\hline Parameter & Df & Sum of Sq & Mean Sq & F Value & $\operatorname{Pr}(\mathrm{F})$ \\
\hline \hline Crossover & 14 & 54420.8 & 3887.20 & 93.4536 & 0.0000000 \\
\hline Mutation & 14 & 162014.1 & 11572.44 & 278.2172 & 0.0000000 \\
\hline Interaction & 196 & 50461.5 & 257.46 & 6.1896 & 0.0000000 \\
\hline Block & 4 & 77.3 & 19.31 & 0.4643 & 0.7619715 \\
\hline Residuals & 896 & 37269.1 & 41.59 & - & - \\
\hline
\end{tabular}

Residual standard error: 6.449417, Estimated effects are balanced. 
Paralleling the results for F2, both crossover and mutation were highly statistically significant together with the interaction. As before, strong statistical significance for the interaction parameter meant that no further increments in sample size were necessary.

Inspection of the sum of squares partitioned into orthogonal polynomial contrast terms as shown in Table A-15 demonstrated up to quadratic behaviour for crossover with the linear component being predominant while for mutation up to cubic behaviour with the quadratic effect being predominant. Interaction was more complex than for F2 with significant interaction terms: linear:linear, quadratic:linear, linear:quadratic and linear:cubic.

Again using polynomial regression with appropriate interaction terms, the overall 3dimensional equation for the response curve was obtained and is given in Table A-19. Figure 9a illustrates the overall 3-dimensional response curve and Figures 9b and 9c illustrate 2-dimensional slices corresponding to crossover and mutation, respectively.

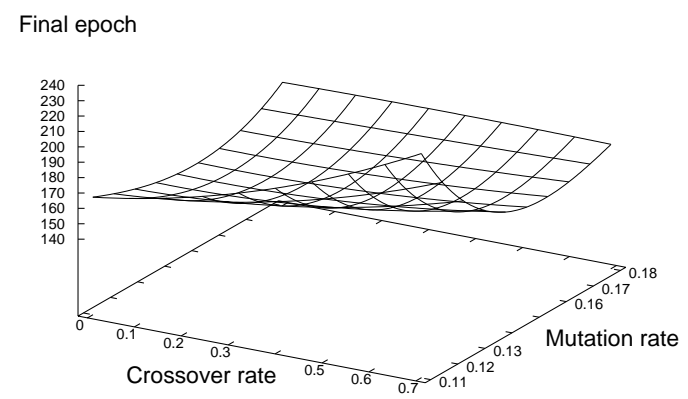

Figure 9a: Fitted response curve: F6.

In Figure 9c we see the curved trend of each mutation curve. However, Figure 9d, which displays mutation curves for crossover rates of $0 \%$ and $10 \%$ respectively illustrates that performance was predicted to improve very slightly with the latter crossover rate of $10 \%$. This was also seen when examining mutation rates for crossover rates of $5 \%$ and $15 \%$. However, to assess in a practical fashion if these differences would be apparent in a data-set focusing upon this range we generated 


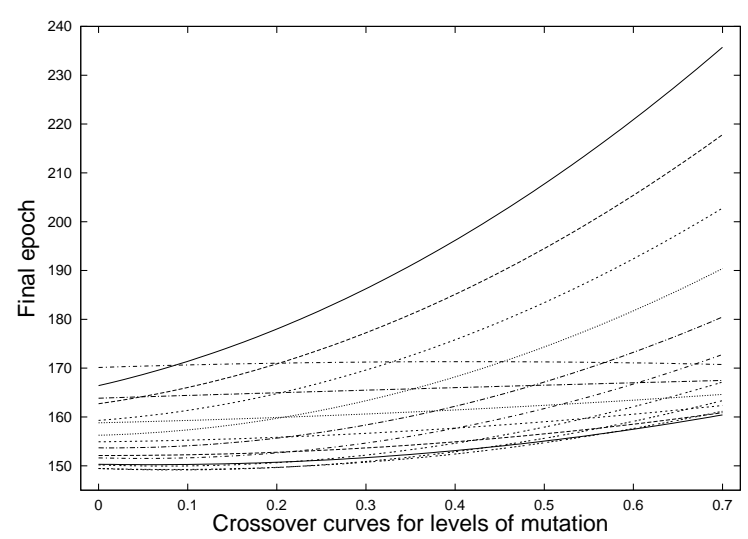

Figure 9b: Fitted response curve: F6-crossover.

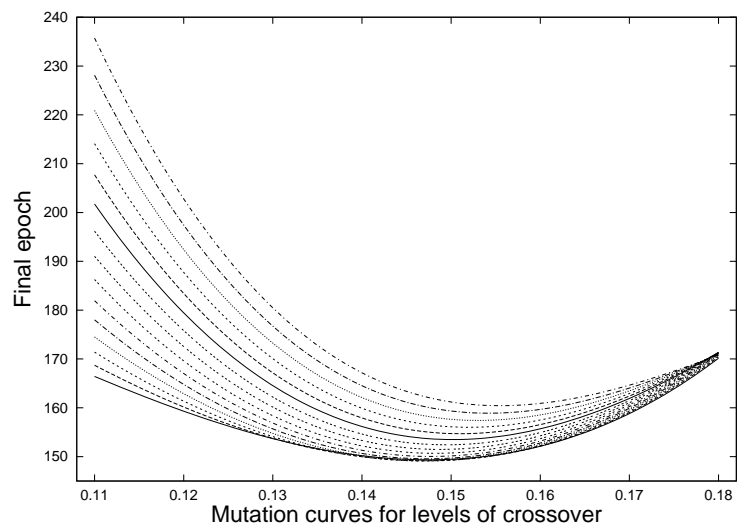

Figure 9c: Fitted response curve: F6-mutation.

five 500 replicate data-sets keeping the mutation range the same but narrowing the range of crossover from $0 \%$ to $15 \%$ inclusive.

As shown in Table A-18 ANOVA analysis illustrated that the differences in performance due to crossover over this range were marginal with a p-value of 0.0208 despite the power being high at $91.63 \%$. Moreover, the partitioned sum of squares illustrated that the effect of crossover was solely linear with a p-value of 0.0003 . Regression analysis confirmed that the coefficient for the linear term was positive indicating a worsening of performance as the crossover rate increased.

Thus, using the equation where the rate of crossover was $0 \%$ the best value for mutation was calculated. The overall results of the analysis are shown in Table 16 . 


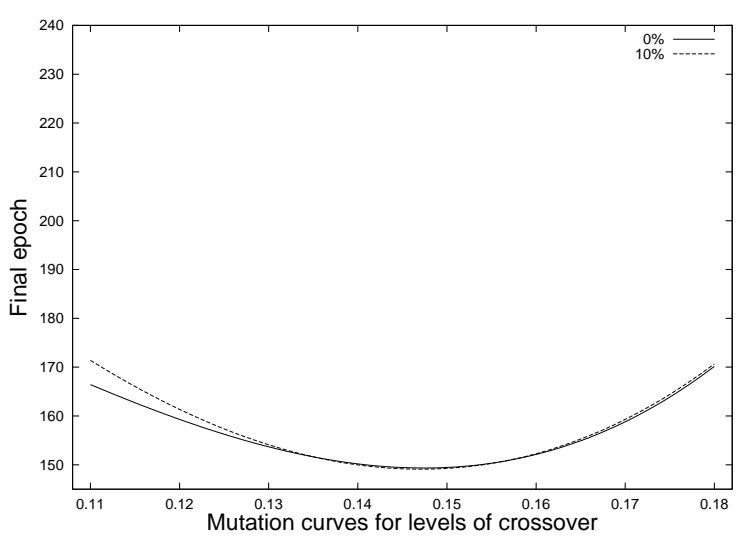

Figure 9d: Fitted response curves for crossover 0\% and 10\%: F6-mutation.

Table 16: F6-Overall results for crossover and mutation.

\begin{tabular}{|c|c|c|c|}
\hline Parameter & Response curve shape & Estimated best value & $99 \%$ CI \\
\hline \hline Crossover & Quadratic & $0 \%$ & - \\
\hline Mutation & Cubic & $15.01 \%$ & $14.80 \%-15.22 \%$ \\
\hline Interaction & Linear:Linear & - & - \\
& Quadratic:Linear & - & - \\
& Linear:Quadratic & - & - \\
& Linear:Cubic & - & - \\
\hline
\end{tabular}

\subsection{Discussion}

Genetic algorithms have been studied in computer science and used in real world applications to find solutions to difficult problems. However, there is no generally accepted methodology to assess which parameters significantly affect performance, whether these parameters interact and how performance varies with respect to changes in parameters. This chapter describes a statistical methodology for the exploratory study of genetic and other adaptive algorithms addressing these issues. Generically, once the algorithm and the problem domain have been specified, the steps in the analysis are:

1. Identify sources of variation and modify the algorithm to generate blocked runs. 
2. Use a workup procedure to minimize the appearance of censored observations and to finalize starting ranges for parameters.

3. Generate an initial data-set consisting of an arbitrary number of replicates. Typically, we have found 100 replicates to be a useful starting point.

4. Calculate power post hoc based upon a chosen effect size. If at least $80 \%$ power is not achieved and the experiment resulted in observing no interaction increase the sample size.

5. Conduct (pooled) ANOVA analysis and determine which parameters are statistically significant.

6. For parameters which are statistically significant partition the sum of squares into polynomial contrast terms. Determine which polynomial terms are statistically significant.

7. Use polynomial regression to obtain the coefficients for the overall response curve (if the interaction parameter is statistically significant) or to obtain the coefficients for the response curve for each parameter separately (if the interaction parameter is not statistically significant).

8. Differentiate and solve the response curve for each parameter to obtain best values and calculate confidence intervals.

Before discussing the specific results of our study it should be prefaced that the present research aimed to provide a statistical methodology by demonstrating its practical use in well known test functions. In this regard, the number of parameters and the suite of problems is restricted. Further research using a statistical approach with an expanded set of parameters, in both continuous and discrete problem domains, will be necessary to expand upon these initial findings.

The analysis of $F 1$ illustrates the way in which our methodology was used to make informed decisions when exploring the relationship between crossover and mutation 
on a specified problem. Initially, workup procedures yielded starting ranges for crossover and mutation. ANOVA of an initial data-set of 100 replicates demonstrated a statistically significant effect upon performance of both crossover and mutation with non-significance for the interaction parameter. Attempting to gauge the shape of the response curve plots was compromised by the small sample size. As seen, the width of the simultaneous $99 \%$ confidence intervals made it unclear as to whether the trend for crossover was linear or included higher order components. In contrast, the sum of squares partitioned into terms corresponding to orthogonal polynomial contrasts demonstrated predominantly linear and quadratic trends for crossover and mutation, respectively. Although this dispelled the ambiguity associated with the data obtained from visual inspection, the subsequent power calculations clearly showed a lack of power for the interaction parameter. Therefore, increases in sample size were required. This was carried out until the appropriate power for the interaction parameter was achieved. At this point polynomial regression was used to obtain fitted response curves and best values with $99 \%$ confidence intervals were calculated.

Looking at the results from the suite of test functions together, crossover appears to have a predominantly linear effect upon performance. For F1 and F3 the positive gradient suggests selecting a rate as high as possible, while for F2 and F6 the negative gradient suggests its possible exclusion. As noted earlier, Schaffer et al [39] documented a relative insensitivity to crossover for these same functions and our research adds to evidence supporting the effectiveness of naive evolution for certain problems. Indeed, as suggested earlier, naive evolution may be a powerful search algorithm in its own right as subtly commented by Eshelman [12]. Given that our study has controlled for the effect of seed we may be obtaining a clearer perspective of the actual behaviour of crossover than has been seen previously. Whatever the case, the observation in our work that crossover appears predominantly linear and that the direction of its slope is problem specific is certainly of practical interest. It may be possible to correlate this behaviour with particular classes of problems 
making it easier to decide how to make the best use of the crossover parameter. This is discussed further in Chapter 5 .

In contrast, mutation appears to have a consistent and predominantly quadratic effect upon performance. Why the effect should be more complex than that of crossover is another question of interest as it may lead to further insights into GA dynamics. The best values of mutation range from $5.11 \%$ to $20.92 \%$ (corresponding to a bit-flipping mutation rate of up to approximately 10\%). These mutation rates add to a growing body of evidence advocating the use of higher mutation rates than have traditionally been used [2]. For example, Petrovski et al [33] who used fractional factorial design followed by regression analysis in order to calculate optimal parameter rates in the domain of cancer chemotherapy reported mutation rates in the range of $10 \%$ to $20 \%$. As with crossover, further statistical work of this kind will assist in the use of the mutation parameter in various problem domains.

The use of statistics also enabled the issue of interaction to be addressed and we found that whether interaction is significant is also problem specific. As to why it is important for some problem domains and not others remains to be answered and may lead to a greater understanding of the interplay between the baseline parameters of crossover and mutation. The kinds of problems for which interaction is significant is further characterized in subsequent chapters.

In conclusion, this chapter has demonstrated a statistical methodology that allows the investigator to undertake exploratory analysis of genetic and other adaptive algorithms. Given the many unique advantages offered by statistical analysis, such as the ability to block for seed, calculation of power and sample size, and rigorous study of response curves, further use of statistics in this exploratory way will assist in the use of GAs as powerful search tools. 


\section{Chapter 3}

\section{The Importance of Interaction}

$\mathcal{1}_{\text {adapting members of a population of potential solutions. Individuals are }}^{\text {s previously discussed, adaptive algorithms such as GAs [6] work by iteratively }}$ adapted through competitive selection mechanisms combined with operators such as crossover and mutation. Since GAs were first developed an important question has been whether crossover and mutation interact or whether each exerts its effect independently in the algorithm.

On the basis of work presented in Chapter 2, particularly for Schaffer's F6, a study was conducted which examined the relationship between the occurrence of interaction between crossover and mutation and increasing modality of a problem. The statistical methodology was applied for assessing the impact of parameter settings and calculating their optimal rates. The results of this work allowed some insight as to when interaction first becomes significant and how this impacts upon the practical task of obtaining optimal rates for crossover and mutation. 


\subsection{Background}

The results of the limited number of studies touching upon the issue of interaction have been conflicting. Petrovski and McCall [32], for example, carried out fractional factorial experiments in the domain of cancer chemotherapy optimization and found only weak interaction between parameters. On the other hand, Schaffer et al [39] conducted a factorial design study which encompassed the De Jong suite and Schaffer's F6, and showed a statistically significant interaction between crossover and mutation which appeared to be function independent.

The difference in the above results may be due to issues such as differing problem domains and the different approaches undertaken. The previous chapter has addressed the limitations of the work of Schaffer et al. In a similar fashion the work of Petrovski and McCall failed to control for the effect of seed, ignored issues dealing with sample size and power, and a detailed analysis of response curves was not considered.

In our own work it was demonstrated that the interaction between crossover and mutation was significant for De Jong's F2 and Schaffer's F6 but not for De Jong's F1 nor De Jong's F3. This led to two important questions.

1. What types of problems are likely to demonstrate statistical significance for the interaction between crossover and mutation?

2. Where interaction between crossover and mutation is statistically significant, what is the practical implication for obtaining optimal rates for these parameters?

In Section 3.2 a brief review is given of the statistical methodology as applied to studying the test functions. The results of this research are then reported in Section 3.3. A discussion in Section 3.4 concludes this chapter. 


\subsection{Methods}

The statistical methodology has already been described in Chapter 2. However, aspects pertinent to this chapter are described below.

\subsubsection{Test Functions}

A generic test function was created, $F N n$, that increases in modality when the integer variable, $n$, is incremented. That is, the function increases in the number of local minima via an increase in peaks and troughs. We formulated this function to elucidate if increasing modality was related to statistical significance for interaction. This was of interest as, particularly for Schaffer's F6 analyzed in Chapter 2, this was a function that was both highly modal and exhibited strong statistical significance for the interaction term. The generic test function, implemented as a minimization problem, is described by Equation 10:

$$
\operatorname{FNn}\left(x_{1}, x_{2}\right)=\sum_{i=1}^{2} 0.5\left(1-\cos \left(\frac{n \pi x_{i}}{100}\right) e^{-\left|\frac{x_{i}}{1000}\right|}\right),-100 \leq x_{i} \leq 100
$$

The test functions for $n=1$ and $n=6$ are shown in Figure 10a and Figure 10b, respectively.

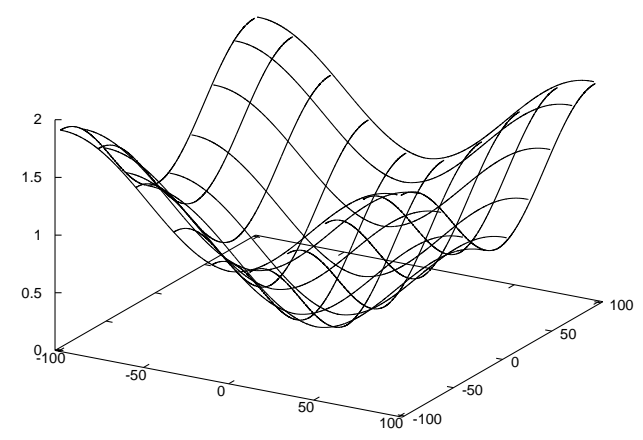

Figure 10a: Test function FN1.

The research consisted of statistical analysis of test functions FN1 to FN6. 


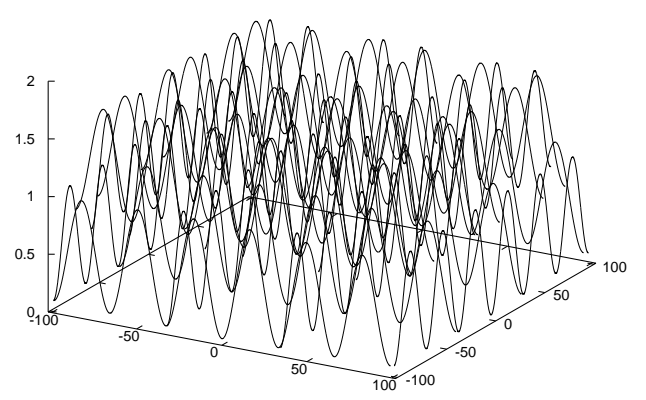

Figure 10b: Test function FN6.

\subsubsection{Power}

Previous work in this thesis has been based on increasing the sample size by a factor of 5 until at least $80 \%$ power is achieved for detecting a difference of at least 5 epochs. However, as $f$ is related to the standard deviation, which may differ considerably according to the problem under study, the previous methodology was refined by calculating power based on an accepted standard value of $f$.

In the previous research the simplest benchmark problem was De Jong's F1 [9] which showed the smallest standard deviation. In reference to this problem a difference of at least 5 epochs was approximated by an $f$ value of 0.4 which denotes a large effect [5]. To obtain a power of at least $80 \%$ using this $f$ value a pooled ANOVA analysis was required (see below) using 5 by 500 replicate data-sets. Therefore 5 by 500 replicate data-sets were used as a starting point in the current study and the level of power achieved for each function was confirmed. The level of power achieved for each function exceeded $80 \%$ except for FN2 where the power using 5 by 500 replicate data-sets was $75.3 \%$. Thus, for FN2 the pooled ANOVA analysis comprised 6 by 500 replicate data-sets where the power achieved was $88.2 \%$.

As the present study was exploratory in nature and a priori assumptions about the standard deviation could not be made we again strictly adhered to post hoc power calculations. 


\subsection{Results}

\subsubsection{ANOVA Analysis of Test Functions}

The results of ANOVA analyzes of pooled results are shown in Table B-1, Table B2, Table B-3 and Table B-4. Analyzes are carried out around the region of best performance in each case.

The effects of crossover and mutation were statistically significant for all test functions. For test functions FN1 to FN4 there was no highly significant effect of interaction between crossover and mutation testing at the $1 \%$ level of statistical significance. However, FN3 with a p-value of 0.011 was marginally significant despite the fact that the function above it in the series, being $F N_{4}$ which is higher in modality, was not statistically significant. This anomaly is explored further in Chapter 4.

By test function FN5 high statistical significance for the interaction between crossover and mutation had been demonstrated at the $1 \%$ level of significance. This continued for FN6.

\subsubsection{Polynomial Regression Analysis of Test Functions}

The results of polynomial regression analyses of pooled results are shown in Table B6 and Table B-7.

For functions $F N 1$ to $F_{4} 4$ and $F N 6$ the response curve for crossover was linear. As the coefficient calculated from polynomial regression for each of these was negative this corresponded to an optimal rate of $100 \%$.

In the case of FN5 the effect of crossover was quadratic. As seen in Figure 11 a crossover rate of $100 \%$ appeared to yield the best performance. In keeping with our previous methodology to verify this we generated 5 by 500 replicate data-sets keeping the mutation range the same but narrowing the range of crossover from 


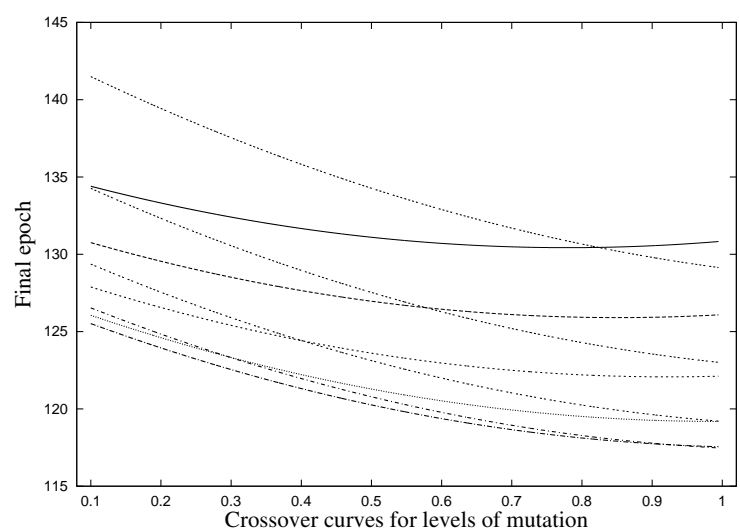

Figure 11: Fitted response curves: FN5-crossover.

Table 17: ANOVA results of crossover $80 \%$ to $100 \%$ for FN5.

\begin{tabular}{|c|c|c|c|c|c|}
\hline \multicolumn{7}{|c|}{ Test function $\boldsymbol{F N 5}$} \\
\hline Parameter & Df & Sum of Sq & Mean Sq & F Value & p-value \\
\hline Crossover & 20 & 127.67 & 6.383 & 0.8516 & 0.6501430 \\
\hline Mutation & 8 & 18725.30 & 2340.663 & 312.2800 & 0.0000000 \\
\hline Interaction & 160 & 1250.80 & 7.817 & 1.0430 & 0.3558088 \\
\hline Block & 4 & 101.05 & 25.262 & 3.3703 & 0.0095623 \\
\hline Residuals & 752 & 5636.54 & 7.495 & - & - \\
\hline \multicolumn{7}{|c|}{ Residual standard error: 2.737773, Power $=99.96 \%}$. \\
\hline
\end{tabular}

$80 \%$ to $100 \%$ inclusive. Table 17 shows that the p-value for crossover was 0.65 illustrating no statistical difference in performance from a crossover rate other than 100\%. Therefore, 100\% was accepted as the optimal crossover rate for FN5.

The optimal rates for mutation for functions FN1, FN2 and FN4 were in the range of $8 \%$ to $9 \%$ (bit-flipping mutation rate in the range of $4 \%$ to $4.5 \%$ ). For FN3, FN5 and FN6 the rates appeared higher in the range of $14 \%$ to $20 \%$ (bit-flipping mutation rate in the range of $7 \%$ to $10 \%$ ). Thus, it also appeared that a relationship existed between the difficulty of the problem and the optimal mutation rate for that problem. That is, the more difficult the problem the higher the optimal mutation rate. The rates themselves compared favourably to other published work that has 
used a statistical approach to calculate the optimal mutation rate, such as the work of Petrovski et al [33] discussed earlier in Chapter 2.

\subsubsection{Polynomial Regression Graphs of Test Functions FN5, FN6}

For FN5 and FN6, the interaction between crossover and mutation was statistically significant and polynomial regression allowed response curves to be generated. The overall response curves are shown in Figure 12a and Figure 12b.

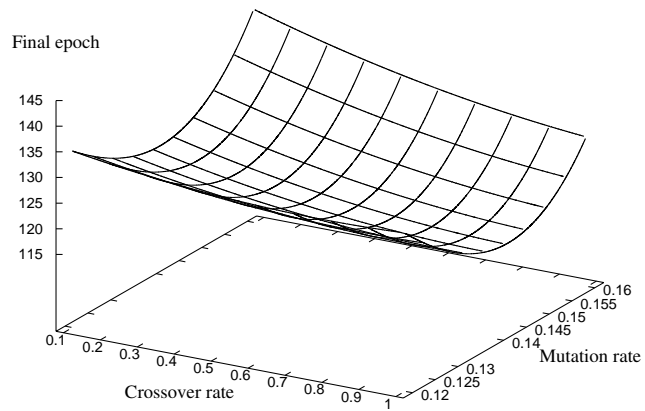

Figure 12a: Fitted response curve: FN5-overall.

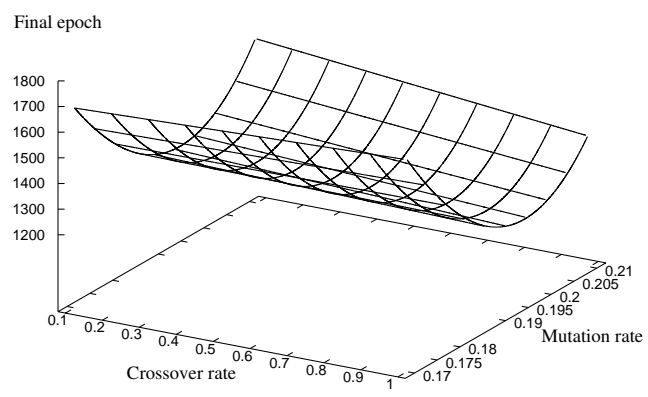

Figure 12b: Fitted response curve: FN6-overall.

The striking effect of interaction upon the relationship between crossover and mutation is best illustrated when viewing the fitted response curves for mutation for 
individual levels of crossover as shown in Figure 13a and Figure 13b.

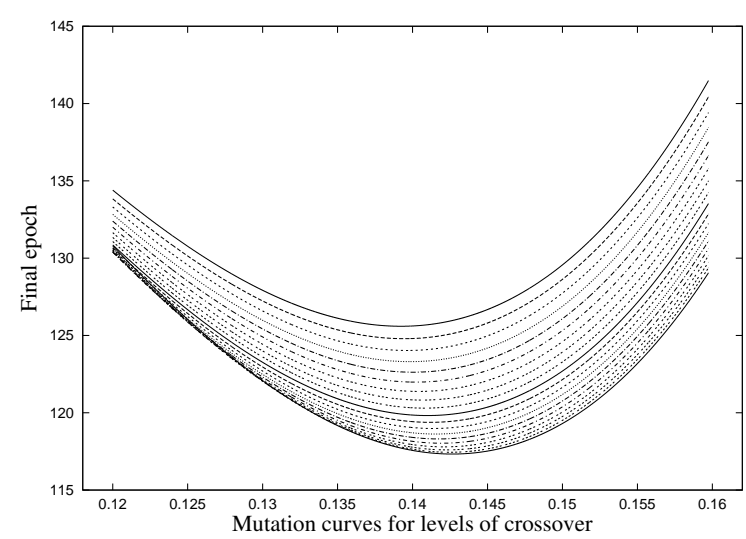

Figure 13a: Fitted response curves FN5-mutation.

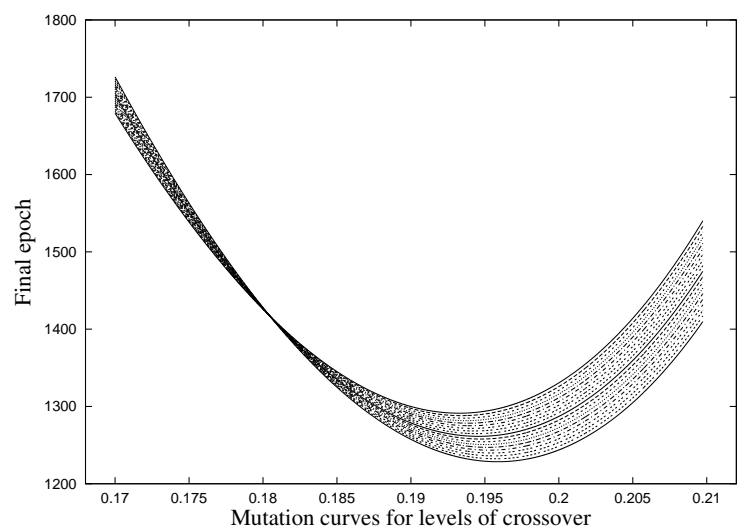

Figure 13b: Fitted response curves FN6-mutation.

For FN5 the left hand side of the curves shows a minor degree of inflection just beginning to occur. For FN6 inflection is well defined and occurs succinctly about a mutation rate of approximately $18 \%$ (bit-flipping mutation rate of approximately $9 \%$ ). The practical implication of these curves is that if attempting to find the optimal rate for crossover and mutation independently, without regard for the effect of interaction, it is uncertain whether the rates obtained will be optimal. For example, optimizing for crossover first using a fixed nominal mutation rate will select a particular curve. Subsequently optimizing mutation will only find a minimum on that particular curve which may differ from the global minimum. In order to allow for the effect of interaction between crossover and mutation each unique combination 
of these parameters, within given starting ranges, must be assessed. An interesting observation from this component of the research was that some problems with lower modality appeared more difficult to solve than problems with higher modality.

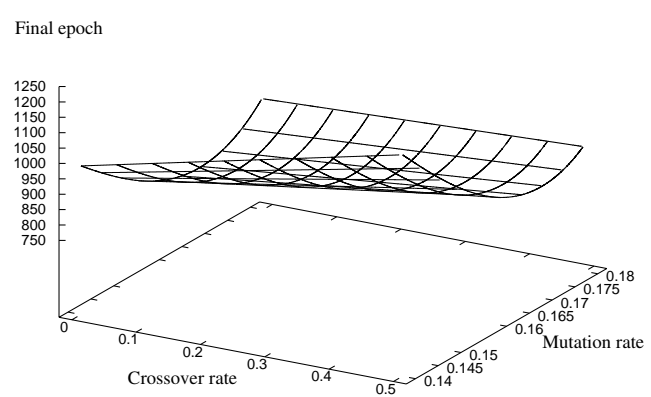

Figure 14a: Fitted response curve: FN3-overall.

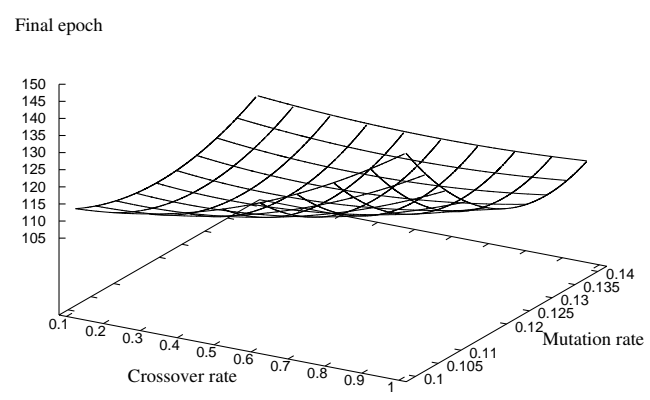

Figure 14b: Fitted response curve: FN4-overall.

Specifically this is illustrated by examining the response curve for FN3 shown in Figure 14a as opposed to FN4 shown in Figure 14b. As can be seen, FN3 proved the more difficult problem to solve despite the fact that it is lower in modality. Moreover, the optimal mutation rate for FN3 was $17.45 \%$ (bit flipping mutation rate of $8.72 \%$ ) while that for FN4 was $8.41 \%$ (bit flipping mutation rate of $4.20 \%$ ). As a high mutation rate appears to be a marker for the difficulty of a problem this added to the evidence supporting the conjecture that FN3 was a more difficult problem to solve than FN4. This observation is explored in greater detail in Chapter 4. 


\subsection{Discussion}

When GAs were first developed they represented a novel approach towards optimization in both continuous and discrete problem domains based primarily on two naturally inspired operations, crossover and mutation. However, a yet unanswered question has been whether crossover and mutation interact or whether each parameter exerts its effect independently during the running of the algorithm. Given the many unique advantages offered by statistical analysis, such as the ability to block for seed, calculation of power and sample size, and rigorous study of response curves, the use of statistical methodology is best suited for this exploratory work. The limited number of statistical studies which have provided data on this topic have been conflicting. However, if interaction does in fact exist between crossover and mutation, this leads to two questions. First, what type of functions are likely to demonstrate interaction between crossover and mutation, and, secondly, what is the practical implication of interaction when attempting to obtain optimal rates for these parameters. An initial attempt to provide answers to these two questions has been made in this chapter by examining the relationship between the occurrence of statistically significant interaction among crossover and mutation and increasing modality of a problem.

Addressing the first question we find that within the class of test functions examined, functions with increased modality are more likely to demonstrate interaction between crossover and mutation. As modality increased beyond $F N_{4}$ the interaction between crossover and mutation was statistically significant. It is conjectured that when dealing with highly modal functions the possibility of interaction must be considered. For simple functions, with low modality, the present research suggests that crossover and mutation are exerting their respective effects independently.

Addressing the second question it has been shown that if interaction is occurring between crossover and mutation attempting to optimize the rate of each parameter independently may result in rates for crossover and mutation which are not optimal. 
In order to account for the effect of interaction all combinations of crossover and mutation, within given starting ranges, must be trialed.

In conclusion, the research in this chapter has made an initial attempt to address the importance of the interaction between crossover and mutation in GAs. Further work of this kind, based on statistical methodology, will afford better insights into the dynamics of GAs. 


\section{Chapter 4}

\section{The Influence of Gray Encoding}

\subsection{Background}

$\mathrm{n}$ integral part of a GA is the type of knowledge representation that is used.
Traditionally, this has been bit encoding with variations such as binary or Gray encoding. Though the operators such as selection and mutation have been studied in some detail, comparatively less formal research has been conducted into the type of knowledge representation that has been implemented.

GA practitioners have reported that changing the representation which is used in GAs affects their performance $[6,37]$. The ability to better understand the influence of intrinsic factors in a GA such as the type of encoding used to represent potential solutions is therefore a major topic of interest.

In the previous chapters interesting results were observed. Firstly, it was noted that for difficult problems (problems with higher modality) increased mutation rates are required. Secondly, as a problem became more difficult, due to increased modality (more local optima), it is generally more likely to demonstrate highly statistically significant interaction between crossover and mutation. 
An unexpected result was that certain problems in our FNn test function series appeared more difficult to solve despite the fact that they have lower modality. Specifically, FN3 appeared a more difficult problem to solve than FN4. This is in contrast to the trend of this test series of increasing difficulty with increasing modality.

This finding led to two important questions which we sought to investigate, building upon the work presented in the previous chapters:

1. Is there a demonstrable relationship between the difficulty of a problem and the choice of encoding or could any observed change in performance be simply due to the stochastic nature of the GA;

2. If the relationship between the difficulty of a problem and the choice of encoding is demonstrable and is thus a real effect, what is the actual mechanism by which this occurs?

In this chapter we use components of our methodology to demonstrate that the type of encoding used can have a real affect upon the difficulty of a problem. Animation is then used to illustrate the actual mechanism by which this effect occurs. This is illustrated using test functions FN3 and FN4 from Chapter 3.

In Section 4.2 a brief review is made of the test functions and methodology. The results of the research are then reported in Section 4.3. A discussion in Section 4.4 concludes this chapter.

\subsection{Methods}

A detailed explanation of the statistical methodology can be found in Chapter 2. Aspects most relevant to this chapter are described below. 


\subsubsection{Test Functions}

Again, use was made of the generic test function series, FNn, that increases in modality as the integer variable, $n$, is incremented. That is, the functions increase in the number of local optima. The test function was implemented as a twodimensional (two bit-string) minimization problem as described by Equation 10:

$$
\operatorname{FNn}\left(x_{1}, x_{2}\right)=\sum_{i=1}^{2} 0.5\left(1-\cos \left(\frac{n \pi x_{i}}{100}\right) e^{-\left|\frac{x_{i}}{1000}\right|}\right),-100 \leq x_{i} \leq 100
$$

Test functions FN3 and FN4 are shown in Figures 15a and 15b, respectively. The test function was deliberately formulated as a linear-separable problem to exploit the fact that optimization of such problems by a GA is decomposable into two independent one-dimensional (one bit-string) sub-problems [38].

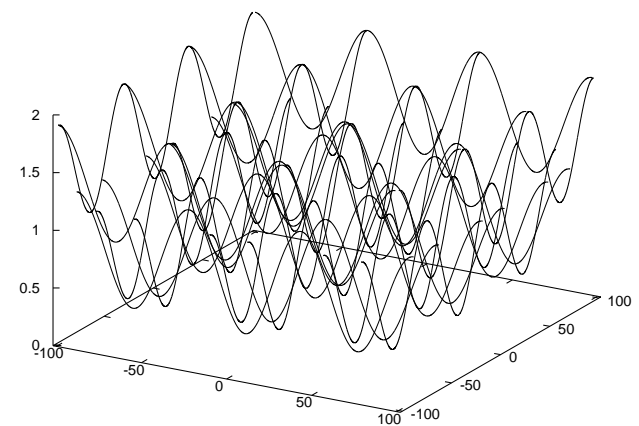

Figure 15a: Test Function: FN3.

\subsubsection{Animation Analysis}

In order to closely study the behaviour of FN3 and FN4 an animation of the GA in solving each function in their one-dimensional (one bit-string) forms was implemented. We were able to visualize the behaviour of the population of chromosomes from epoch to epoch after the processes of selection, crossover and mutation. The previous chapters have shown that the best rate of crossover for FN3 and FN4 is 


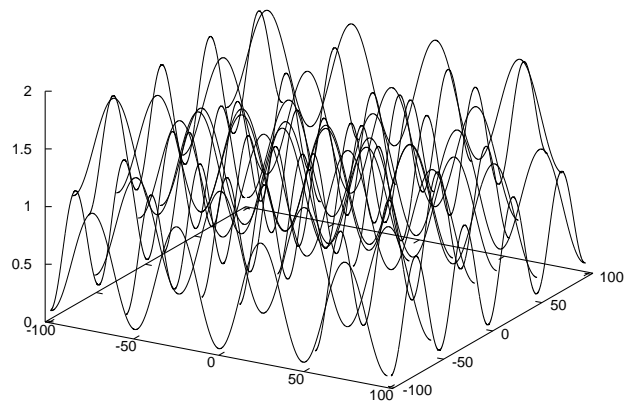

Figure 15b: Test Function: FN4.

$100 \%$. Thus, the study of the behaviour of the chromosomes was carried out by setting crossover at $100 \%$ and varying the rates of mutation in accordance with the results from dot diagram analysis.

\subsection{Results}

\subsubsection{Response Curve Analysis of FN3 and FN4}

As previously discussed in Chapter 3, the number of epochs required to solve the problem, as shown in the response curves, demonstrated that FN3 was the more difficult problem to solve despite it being lower in modality (see Figure 14a and Figure 14b).

\subsubsection{Dot Diagram Analysis of FN3 and FN4}

Dot diagram analysis of FN3 and FN4 are shown in Figures 16a and 16b.

For FN3 mutation rates of $10 \%$ or less were associated with censoring. In contrast, for FN4 low rates of mutation were not associated with censoring. This assessment of the two functions suggested that despite being lower in modality, FN3 was proving a more difficult function to solve than FN4. 


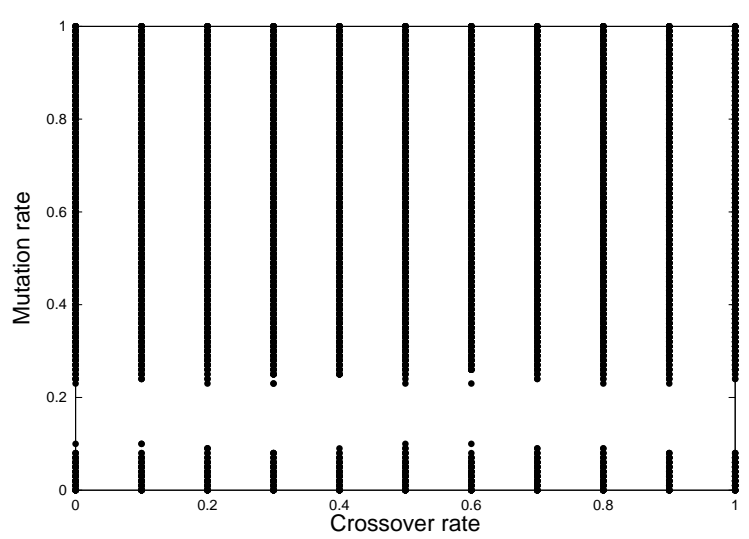

Figure 16a: Dot Diagram: FN3.

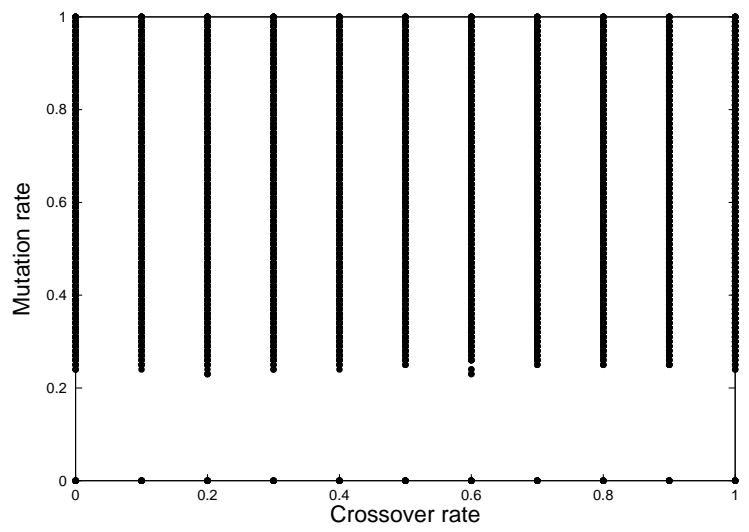

Figure 16b: Dot Diagram: FN4.

\subsubsection{Dot Diagram Analysis of One Dimensional Projec- tions}

In order to explain the above anomaly it was necessary to better understand the interaction between the GA and the solution space. This was achieved by developing a computer animation that allowed observation of the behaviour of individuals as iterations were stepped through.

Visualization of the behaviour is simpler for a one-dimensional (one bit-string) problem. Since our test function is linear separable, its optimization by a GA can be envisaged as decomposable into two independent one-dimensional (one bitstring) sub-problems. Providing those sub-problems exhibit the same phenomenon, 
we can confine our study to their one-dimensional (one bit-string) forms. These are denoted as FN3 $1 D$ and FN41D.

Dot diagram analysis of $F N 3_{1 D}$ and $F N 4_{1 D}$ were undertaken and are shown in Figures $17 \mathrm{a}$ and $17 \mathrm{~b}$. As can be seen, low mutation rates were associated with censoring for $F N 3_{1 D}$, while for $F N 4_{1 D}$ there was an absence of censoring. As these results paralleled those for the two-dimensional (two bit-string) functions we proceeded to study the behaviour of $F N_{1 D}$ and $F N_{41 D}$ via animation.

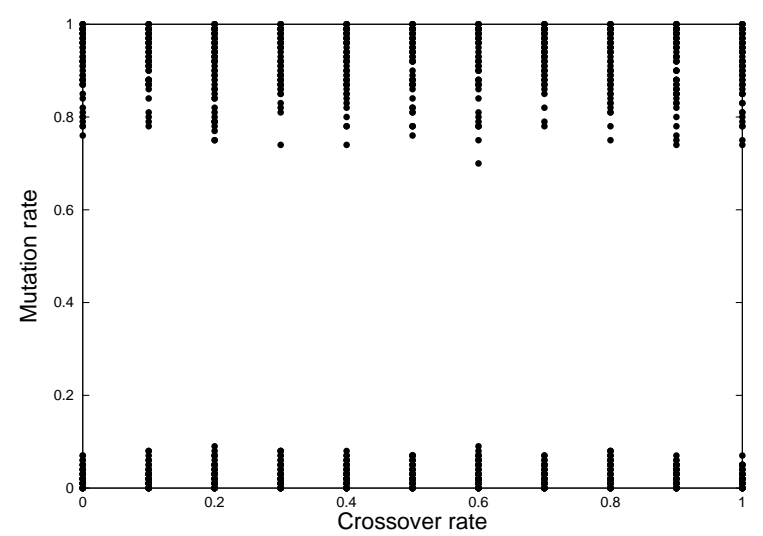

Figure 17a: Dot Diagram: $F N 3_{1 D}$.

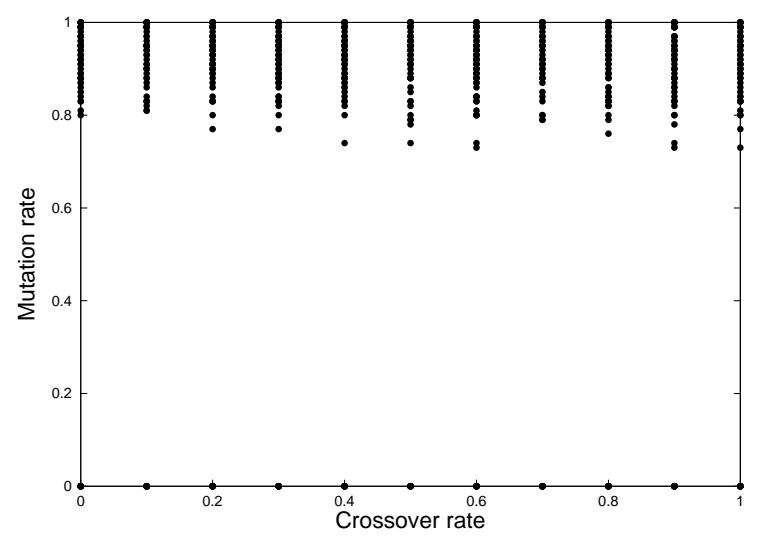

Figure 17b: Dot Diagram: $F N_{41 D}$. 


\subsubsection{Animation Analysis of $F N 3_{1 D}$ and $F N 4_{1 D}$}

The animation analysis of $F N 3_{1 D}$ and $F N 4_{1 D}$ revealed interesting insights into the performance of the GA. Some illustrative examples are discussed below.

As shown in Figure $18 \mathrm{a}$ and Figure $18 \mathrm{~b}$, for $F N_{1 D}$, after applying a low mutation rate a number of chromosomes would lie in the upper part of the "optimal valley" (the valley containing the global optimum). However, after selection these chromosomes would be culled and fail to survive into the next generation.

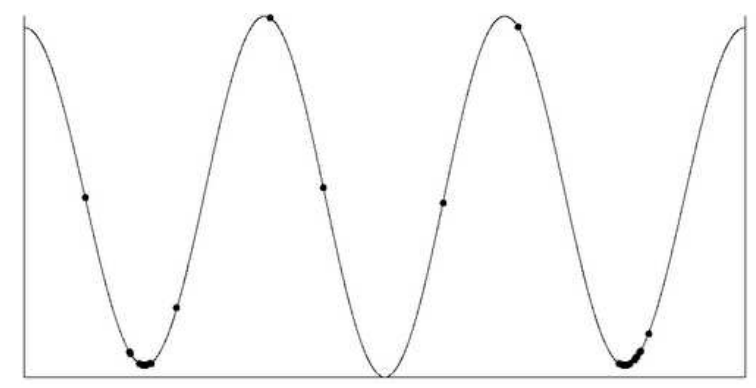

Figure 18a: $F N 3_{1 D}$ : Chromosome population after applying a low mutation rate.

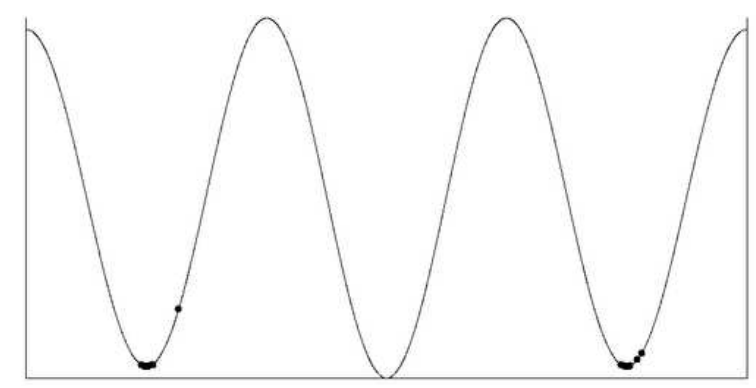

Figure 18b: $F N 3_{1 D}$ : Chromosome population after selection.

In contrast, as illustrated in Figures 19a and 19b, high mutation rates were able to produce chromosomes lying deep enough in the "optimal valley" to survive culling and be selected into the next generation.

Thus, it appeared for $F N 3_{1 D}$ that movement from the local optima to the global optimum was a difficult task that could only be achieved with the use of high mutation rates.

In contrast, for FN41D, again starting outside the global optimum, low mutation 


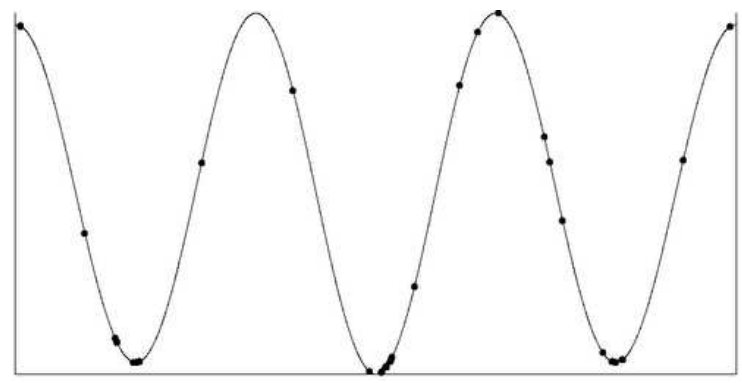

Figure 19a: $F N 3_{1 D}$ : Chromosome population after applying a high mutation rate.

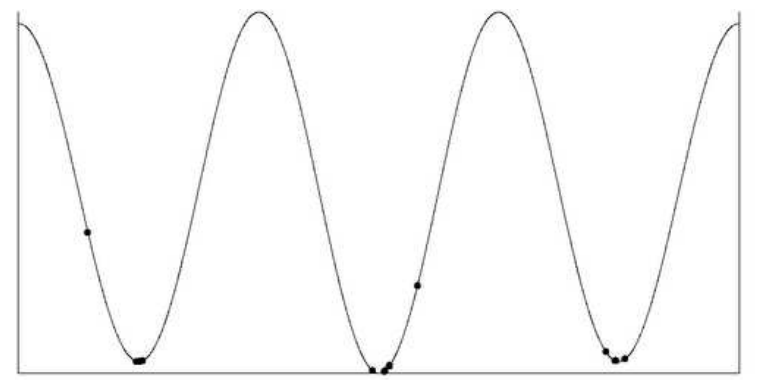

Figure 19b: $F N 3_{1 D}$ : Chromosome population after selection.

rates were able to produce chromosomes lying deep enough in the optimal valley to survive into the next generation. Thus, this movement appeared to be less difficult than for $F N 3_{1 D}$. However, an additional interesting observation from $F N_{41 D}$, as shown in Figure 20a, Figure 20b and Figure 20c, was that chromosomes appeared to move with greater ease again from the outer-most local optima to the local optima adjacent to the global optimum.

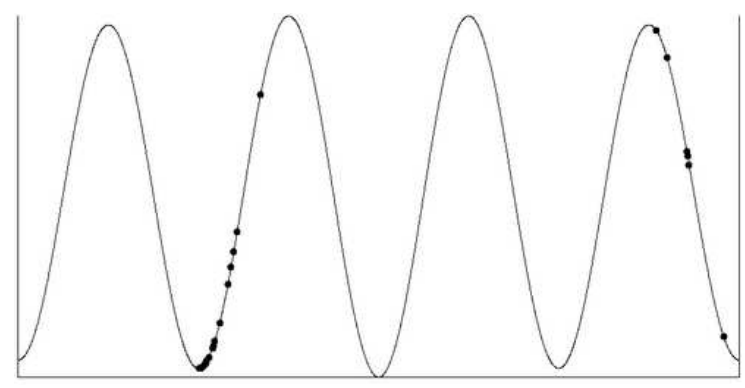

Figure 20a: FN41D: Chromosome population prior to applying mutation.

In overview, the animation revealed that there was a factor causing the chromosomes to move with either greater or lesser difficulty from local optima to the global 


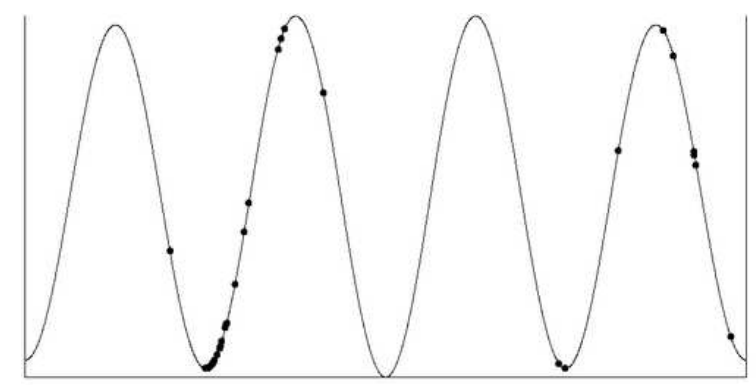

Figure 20b: FN41D: Chromosome population after applying a low mutation rate.

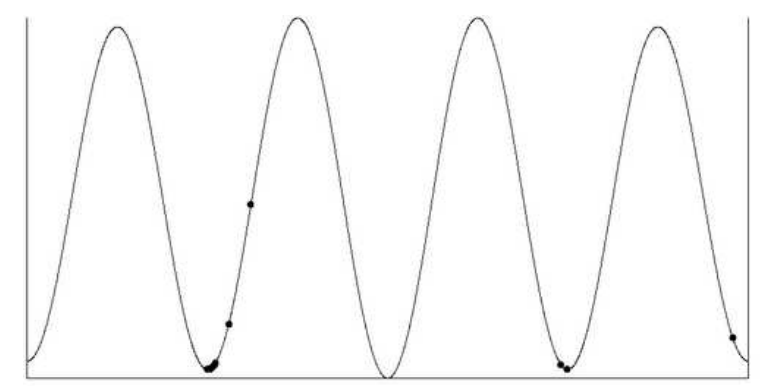

Figure 20c: $F N_{41 D}$ : Chromosome population after selection.

optimum. It was this factor that was making $F N 3_{1 D}$ more difficult for the GA than FN41D.

It was conjectured that the difficulty of jumping between local optima was related to the number of coincident mutations required to make that transition. The probability of a successful jump would therefore reduce with the product of the probabilities of each individual mutation required. To test this hypothesis examination was made of the number of bit changes required to pass between local optima in $F N 3_{1 D}$ and FN41D.

\subsubsection{Hamming Distances for $\boldsymbol{F} N 3_{1 D}$ and $\boldsymbol{F} N 4_{1 D}$}

The number of bit changes required to jump from one bit-string to another is the Hamming Distance of the bit-strings. Gray coding has been proposed as a good encoding for applications such as GAs because the Hamming Distance between any two adjacent solution candidates is one, as compared to binary encoding where all 
bits may change in moving from one decimal integer to the next. The idea is that this allows individuals to explore the solution space via small mutations.

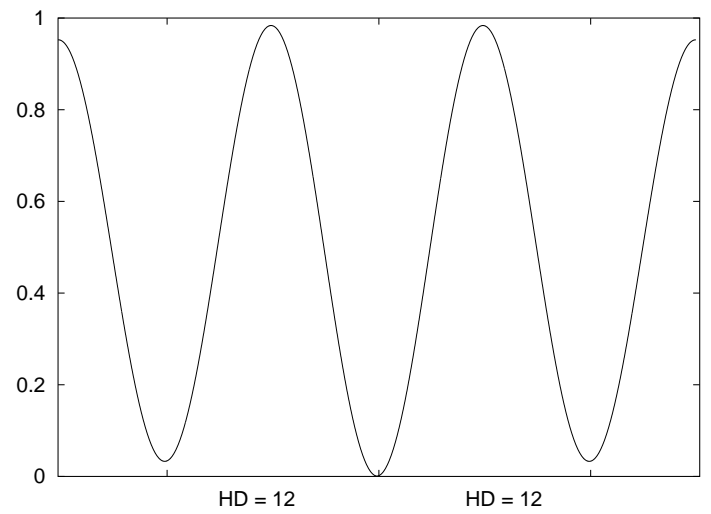

Figure 21a: $F N 3_{1 D}(\mathrm{HD}=$ Hamming Distance).

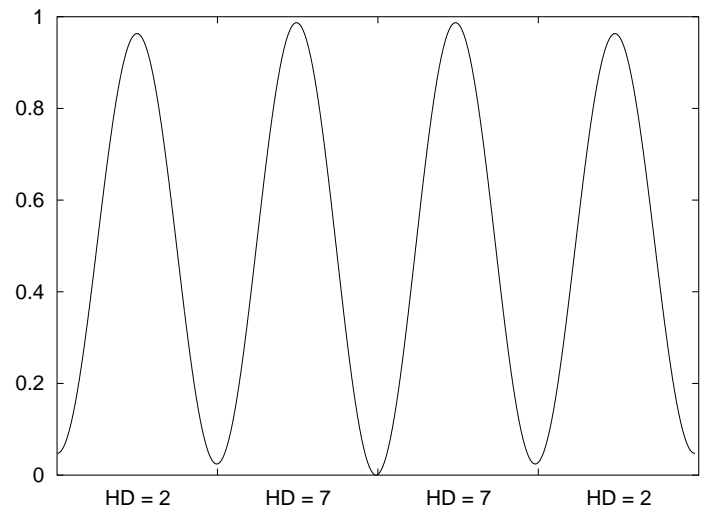

Figure 21b: $F N_{41 D}(\mathrm{HD}=$ Hamming Distance).

To find the mutations actually required for the GA to make progress in solving the multi-modal problems the Hamming Distances between local optima were calculated. For $F N 3_{1 D}$, as illustrated in Figure 21a, the Hamming Distance between the local optima and the global optimum was 12. In contrast for FN41D the Hamming Distance between the local optima adjacent to the global optimum and the global optimum was only 7 . Since mutation probabilities are multiplicative (for example, $0.1^{7}$ versus $0.1^{12}$ ), there existed a much lower probability of chromosomes moving into a sufficiently fit part of the optimal valley to survive selection for $F N 3_{1 D}$ as opposed to FN41D. This explained why higher mutation rates were necessary for 
$F N 3_{1 D}$

Furthermore, for FN41D the Hamming Distance between the outer-most local optima and the local optima adjacent to the global optimum was only 2. Thus, it proved easy for chromosomes to move into the local optima adjacent to the global optimum. Hence, the fact that $F N 41 D$ was more modal than $F N 3_{1 D}$ was of little consequence since the Hamming Distance between these local optima was comparatively small.

In overview, the results demonstrated by the dot diagram analysis, ANOVA, and finally by animation analysis, all consistently demonstrated that FN3 was a more difficult problem than FN4. By computing Hamming Distances it was found that, despite $F N 3_{1 D}$ being of lower modality than $F N_{41 D}$, these Hamming Distances were significantly higher for $F N 3_{1 D}$ making it a more difficult problem. This was a direct result of the relationship between the encoding and the solution space.

\subsection{Discussion}

In respect of the intrinsic factors which may affect GA performance two important questions have been whether there is a significant relationship between the difficulty of a problem and the choice of encoding, and, if so, what is the actual mechanism by which this occurs.

In this chapter the first question has been addressed by showing that a lower modality problem is more difficult to solve with a Gray encoding than a higher modality problem. This is in contrast to the identified trend of problem difficulty increasing with increasing modality. Specifically, response curve analysis and dot diagram analysis suggested that FN3 is a more difficult problem than FN4, despite the fact that FN4 is higher in modality. To investigate this further, since the original functions are linear-separable, our test functions were decomposed into their onedimensional (one bit-string) forms. Subsequent dot diagram analysis confirmed the ability to do so. 
To address the second question animations of the GA in solving each function in their one-dimensional (one bit-string) form were created which clearly demonstrated that the ability of chromosomes to move between local optima and avoid culling in the two functions was significantly different. Movement towards the global optimum was much more difficult in $F N 3_{1 D}$ than for $F N 41 D$.

The probability of a successful jump is dependent on the Hamming Distance. Calculation was therefore made of the Hamming Distances between local optima present in the two functions and it was found that movement within $F N 3_{1 D}$ was more difficult because of the significantly higher Hamming Distances involved. Moreover, even though $F N_{41 D}$ is higher in modality the very small Hamming Distance between the outer-most local optima and the local optima adjacent to the global optimum counteracted the influence of its increased modality. These Hamming Distances are a result of the relationship between the encoding and the shape of the functions. 


\section{Chapter 5}

\section{The Detrimentality of Crossover}

$\mathrm{I}$

t has been traditionally maintained that the crossover operator is an integral component of a GA. This has been held to the extent that some GA researchers believe that it is the inclusion of the crossover operator that distinguishes GAs from all other optimization algorithms [6].

Despite this, work by Eshelman and Schaffer [13], entitled Crossover's Niche, suggested that there exists a unique niche for which crossover is advantageous, and that it is smaller than has traditionally been held in the GA community. Saloman [38] suggested that Crossover's Niche is linear-separable problems. From his work with Rastrigin-like functions he conjectured that crossover implicitly exploits the decomposability property of the fitness function: the optimization is decomposable into $n$ independent one-dimensional (one bit-string) sub-problems. If such a conjecture is true, it adds further to the debate concerning crossover since most problems in the real world are not-linear-separable, but tend to be non-linear, chaotic and stochastic [10].

We explored Salomon's conjecture to see if linear-separability was indeed linked to crossover's niche or whether other factors came into play in rendering the crossover operator detrimental upon GA performance.

In Section 5.1 the literature on the detrimentality of crossover is reviewed. This 
is followed in Section 5.2 by a review of the work in the present thesis on this subject. Section 5.3 briefly reviews the statistical methodology. Next, Section 5.4 and Section 5.6 details the results of our experiments with the latter carrying over to a more difficult practical optimization problem. Section 5.5 reviews the factors affecting the detrimentality of crossover. Section 5.7 concludes this chapter.

\section{$5.1 \quad$ Background}

As discussed above, from a traditional perspective it has been maintained that crossover is a necessary inclusion in a GA. Mutation, on the other hand, has been traditionally seen as a background operator with the unique role, as described by Holland, of ensuring that no allele or value of a bit character ( 0 or 1 ) permanently disappears from the population [20]. However, there is considerable debate with some suggesting that the crossover operator may not always make a useful contribution to GA performance. As Eshelman [12] subtly conjectured, naive evolution (a GA which is composed of selection and mutation only) is a much more powerful algorithm than many people in the GA community have been willing to admit.

The results of research into the detrimentality of crossover have been inconclusive. As discussed above, Eshelman and Schaffer conjectured the idea of crossover's niche. The authors argued that what distinguishes the GA among population-based hillclimbers is pairwise mating and that problems can be devised where crossover is given a competitive advantage. However, as discussed before, many problems do not have these features and it remains an open question as to how important crossover may be for real world problems. In addition, because GAs are susceptible to premature convergence the niche for which crossover is beneficial to GA performance may be smaller than most GA practitioners maintain [13]. Moreover, Reeves and Wright [35] suggested that the amount of information in a sample can never be sufficient to enable one to decide on the amount of epistasis in a problem. This implies that the problems that Eshelman and Schaffer describe as being most apt 
for the crossover operator may not be easily recognizable in practice.

Jones [25] added to this by showing that a macromutational hillclimber (one that involves large scale mutations) easily outperforms a standard GA on Holland's Royal Road problem [29] which has the properties that Eshelman and Schaffer ascribe to problems residing in crossover's niche. Thus, the niche may be even smaller than Eshelman and Schaffer had intended.

Further evidence on the usefulness of crossover was contributed by Fogel and Atmar [15] who conducted several experiments that required solving systems of linear equations. They concluded that the crossover operator provided no significant benefit. Jansen and Wegener [22], on the other hand, proved that the crossover operator can be useful if the current population of strings has a certain diversity. They proved that an evolutionary algorithm can produce enough diversity such that the use of crossover can speed up the expected GA optimization time from superpolynomial to a polynomial of small degree. This was shown only for small crossover probabilities, however, and they remarked that it was an open question as to whether similar results could be shown for more realistic crossover rates [23]. Moreover, they proved [23] that for some explicitly defined fitness function, namely the Royal Road functions, a GA with crossover can optimize in expected polynomial time while all evolutionary strategies based only on mutation (and selection) required exponential time.

Statistical analyses of GA performance have failed to clarify this situation. As discussed previously, Schaffer et al [39] conducted a factorial study using ANOVA to examine the De Jong suite plus an additional five problems. Close examination of the best on-line pools suggested a relative insensitivity to the crossover operator when using Gray encoding. However, again this work did not block for seed, ignored power calculations and was limited in its analysis of response curves.

Thus, in reference to the above studies three important questions were raised:

1. Can the crossover operator be statistically demonstrated to be detrimental for a 
given problem in the first instance?

2. In reference to the work of Salomon, is not-linear-separability a sufficient determinant of the detrimentality of crossover?

3. If not, what other factors are involved?

\subsection{Observations from Earlier Work}

Our previous work with ANOVA involved examination of four benchmark problems. These are displayed again below:

$$
\begin{gathered}
f_{1}(\mathbf{x})=\sum_{i=1}^{3} x_{i}^{2},-5.12 \leq x_{i} \leq 5.12, \\
f_{3}(\mathbf{x})=\sum_{i=1}^{5}\left\lfloor x_{i}\right\rfloor,-5.12 \leq x_{i} \leq 5.12 \\
f_{2}(\mathbf{x})=100\left(x_{2}-x_{1}^{2}\right)^{2}+\left(1-x_{1}\right)^{2},-2.048 \leq x_{i} \leq 2.048 \\
f_{6}(\mathbf{x})=0.5+\frac{\left(\sin \sqrt{x_{1}^{2}+x_{2}^{2}}\right)^{2}-0.5}{\left(1.0+0.001\left(x_{1}^{2}+x_{2}^{2}\right)\right)^{2}},-100.0 \leq x_{i} \leq 100.0 .
\end{gathered}
$$

It was found that for De Jong's F1 and F3 the traditional GA, where crossover was included, performed optimally when the crossover rate was $100 \%$. In contrast for De Jong's F2 and Schaffers F6, the crossover operator was statistically demonstrated to be having a detrimental effect upon performance. It was also found for these latter two functions that the ANOVA interaction term between crossover and mutation was significant and negative, which indicates an inverse relationship between crossover and mutation. Moreover, the difficulty of a problem was associated with the optimal mutation rate, with De Jong's F2 and Schaffer's F6 demonstrating optimal mutation rates significantly higher that traditional recommendations. 
When considering the possible difference in these functions that could produce such varied results a clear demarcation between them was that De Jong's F1 and F3 are linear-separable ${ }^{1}$, echoing the conjecture made by Salomon that linear-separable problems are crossover's niche. In contrast, De Jong's F2 and Schaffer's F6 are not-linear-separable problems. However the functions are also quite different in structure, allowing explanations other than linear-separability.

To address the second question therefore, we compared two test functions differing only in that one test function series was linear-separable while the other was notlinear-separable.

The two test functions we decided to compare comprised firstly of the test function series, FNn, which was used in Chapter 3 to examine the importance of the ANOVA interaction term between crossover and mutation. This is a linear-separable problem which increases in modality as the value for $n$ increases.

The second test function series consisted of the same functions rotated by 45 degrees in the solution space. This rotation rendered the series of problems, which we call FNnR45, not-linear-separable.

By comparing the linear-separable form of the problem to the not-linear-separable form we expected to see a difference in the effect of the crossover operator. Given the suggestions from the literature and previous experience with linear-separable versus not-linear-separable functions, it was conjectured that we would observe a largely beneficial effect of crossover for the linear-separable problems, $F N n$, but a detrimental effect for the not-linear-separable problems, FNnR45. Furthermore, if the latter turned out to be true, then an attempt would be made to explain the reasons why crossover acts detrimentally for not-linear-separable problems.

Finally, given the conjecture by Eshelman and Schaffer that it remains an open question as to how important crossover may be for real-world problems [13] the

\footnotetext{
${ }^{1}$ We define linear-separable problems as those where the objective function can be written as a sum of univariate functions, which are allowed to be not-linear, where each of the functions can take one component of the input vector as an argument.
} 
GA was trialed on a practical (but still highly multimodal) landscape minimization problem to see if the results from the test functions would carry over to those obtained on the real world landscape.

\subsection{Methods}

Our statistical methodolgy has been discussed in the previous chapters. Here we focus on some aspects of the experimental setup for this particular chapter.

\subsubsection{Motivation for our Test Functions}

As discussed, to determine whether linear-separability is indeed a determining factor while minimizing other effects, we examined a series of functions of increasing difficulty, while also examining the same functions in different orientations (that is, the only difference was the frame of reference). We achieved this by rotating the functions by 45 degrees rendering them not-linear-separable. We then tested the algorithm on a newly devised benchmarking problem from the Huygens Suite [28]. These functions are detailed below:

1. Test functions $F N n$ for $\mathrm{n}=1$ to $\mathrm{n}=6$, which are linear-separable equations, as displayed in Equation 10 below:

$$
F N n\left(x_{1}, x_{2}\right)=\sum_{i=1}^{2} 0.5\left(1-\cos \left(\frac{n \pi x_{i}}{100}\right) e^{-\left|\frac{x_{i}}{1000}\right|}\right),-100 \leq x_{i} \leq 100 .
$$

2. Test function $F N n R 45$ ( $R 45$ standing for the original test function $F N n$ having been rotated by 45 degrees in the solution space), being not-linearseparable, for $\mathrm{n}=1$ to $\mathrm{n}=6$ as displayed in Equation 11 below: 


$$
\begin{gathered}
F N n R 45\left(x_{1}, x_{2}\right)=0.5\left(1-\cos \left(\frac{n \pi \frac{x_{1}+x_{2}}{\sqrt{2}}}{100}\right) e^{-\left|\frac{\frac{x_{1}+x_{2}}{\sqrt{2}}}{1000}\right|}\right)+ \\
\quad 0.5\left(1-\cos \left(\frac{n \pi \frac{x_{1}-x_{2}}{\sqrt{2}}}{100}\right) e^{-\left|\frac{\frac{x_{1}-x_{2}}{\sqrt{2}}}{1000}\right|}\right),-100 \leq x_{i} \leq 100
\end{gathered}
$$

3. MacNish has devised a problem series for benchmarking, that based on fractal landscapes, reflect the attributes of highly multimodal problems seen in real world situations $[27,28]$. We chose to run our GA on the first landscape in MacNish's 20 series for which a plot was provided, shown in Figure 22.

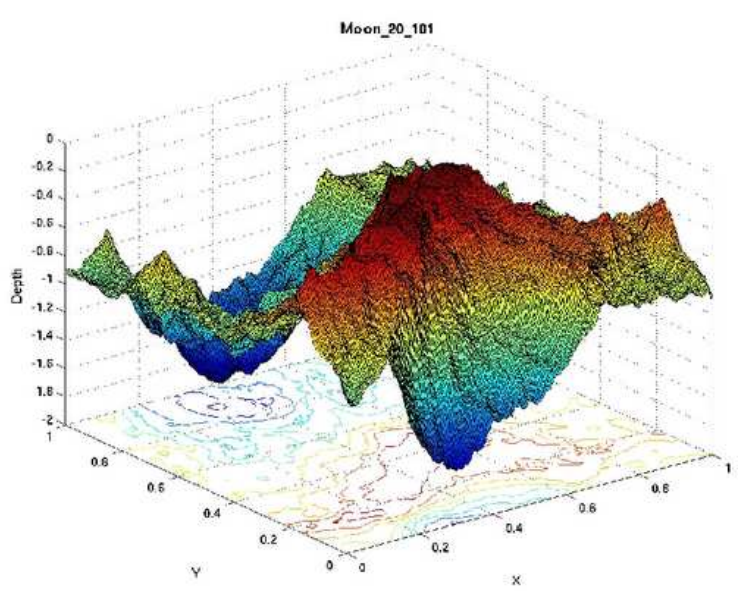

Figure 22: Landscape 20_101 from the Huygens Suite.

\subsubsection{Power}

As outlined previously it is imperative to have some means of calculating whether the size of the sample chosen has sufficient power. In order to do so it is necessary to specify the degree to which the null hypothesis is false. This can be done by using the effect size index, $f$, as described by Cohen [5]. 
As $f$ is related to the standard deviation, which may differ considerably according to the problem under study, we again refined our previous methodology by calculating power based on an accepted standard value of $f$.

Given the previous experience in power calculations with GA analysis, a value of 0.4 was utilized as a standard for the effect size when attempting to analyze the performance of a GA. It should also be noted that in using this approach it is possible to calculate power a priori and thus ascertain if a given sample size will confer a required level of power. However, in this chapter we continued to adhere to post hoc power calculations in line with the work of the previous chapters.

\subsubsection{Estimates of Optimal Values for Crossover and $\mathrm{Mu}-$ tation}

The aim of the present research was to explore the detrimentality of crossover. That is, to statistically determine the optimal crossover rate for each test function with detrimental crossover corresponding to an optimal crossover rate of $0 \%$. Therefore, use was made of previous described methodology which enlisted polynomial regression to obtain an estimate of the optimal rate for both crossover and the mutation operators.

\subsection{Results}

\subsubsection{Exploratory Analysis of Test Functions FN1 to FN6}

Full ANOVA tables and regression analyses for test functions FN1 to FN6 are to be found in Table B-1 to Table B-7. The results showed that the crossover operator proved beneficial to the performance of the GA in every instance: Table B-6 and Table B-7 show that the optimal value of crossover was $100 \%$ for each of the six functions. 


\subsubsection{Exploratory Analysis of test functions FN1R45 to FN6R45}

ANOVA tables and regression analyses for test functions FN1R45 to FN6R45 are shown in Table C-1 to Table C-7. For the test function series, FNnR45, where the test function $F N n$ had been rotated by 45 degrees in the solution space, there was a marked difference in the results obtained.

Firstly, crossover was detrimental for test functions FN2R 45, FN4R45 and FN5R45, where for these rotated forms the optimal crossover rate was $0 \%$. This is in contrast to the non-rotated form of these functions, as described above, where in each case crossover proved to be beneficial. By contrast, crossover was beneficial for FN1R45, FN3R45 and FN6R 45. This shows that linear-separability alone is not a sufficient indicator for the detrimentality of crossover.

Also, where crossover was shown to be detrimental the mutation rate was also higher than in instances where crossover was having a beneficial effect. For example, for FN2R45 the optimal mutation rate was $25.45 \%$ (bit flipping mutation rate of $12.72 \%$ ), for $F_{4} R_{4} 5$ the optimal mutation rate was $35.30 \%$ (bit flipping mutation rate of $17.65 \%$ ) and for $F N 5 R 45$ the optimal mutation rate was $33.38 \%$ (bit flipping mutation rate of $16.69 \%$ ). In contrast, for FN1R45 the optimal mutation rate was $8.78 \%$ (bit flipping mutation rate of $4.39 \%$ ), for FN3R45 the optimal mutation rate was $12.36 \%$ (bit flipping mutation rate of $6.18 \%$ ) and for FN6R45 the optimal mutation rate was $12.97 \%$ (bit flipping mutation rate of $6.48 \%$ ). Thus, in all cases where crossover was detrimental the optimal mutation rate proved to be notably greater than those instances where crossover was beneficial. These mutation rates also reflected those obtained from the literature when a statistical approach was adopted [33].

As noted above, as a high mutation rate is a conjectured marker for the difficulty of a problem the above results indicate that the crossover operator proved to be detrimental for the most difficult of the not-linear-separable rotated functions. 


\subsection{Factors Affecting the Detrimentality of Crossover}

In the preceding work it was demonstrated that crossover was detrimental for three of the six not-linear-separable rotated functions analyzed. As indicated by the optimal mutation rates, these proved to be the most difficult of the six functions to solve. Thus, it is conjectured that crossover proves to have a detrimental effect upon GA performance if the not-linear-separable problem is difficult for the GA to solve.

What makes a GA hard to solve is a complex issue and involves factors such as the degree of optimization occurring at local minima due to crossover, the bias of the mutation operator and the Hamming Distances involved in the individual problems. In the next sections each of these factors is discussed in turn.

\subsubsection{Optimization Occurring at Local Minima due to Crossover}

The first factor which influenced the difficulty of the problem for the GA was the optimization occurring at local minima due to crossover. In order to discuss this an investigation must firstly be carried out to determine what roles crossover, and also mutation, are playing in the GA.

Figure 23a, Figure 23b, and Figure 23c show examples of chromosomes situated in a heat map of function FN2R45. The heat map represents a view of the function looking down from above with white areas denoting troughs and dark areas denoting peaks. These heat maps show the location of the 50 chromosomes during iterations of the GA to enable one to gain a pictorial understanding of their behaviour.

Figure 23a shows a population taken from a random epoch while solving FN2R45 (note that some chromosomes are occluded).

Figure 23b, shows the location of the chromosomes after crossover. The chromosomes have dissipated little, moving by only a small amount at the local minima sites (denoted by the white areas). Crossover is performing its classical function of 


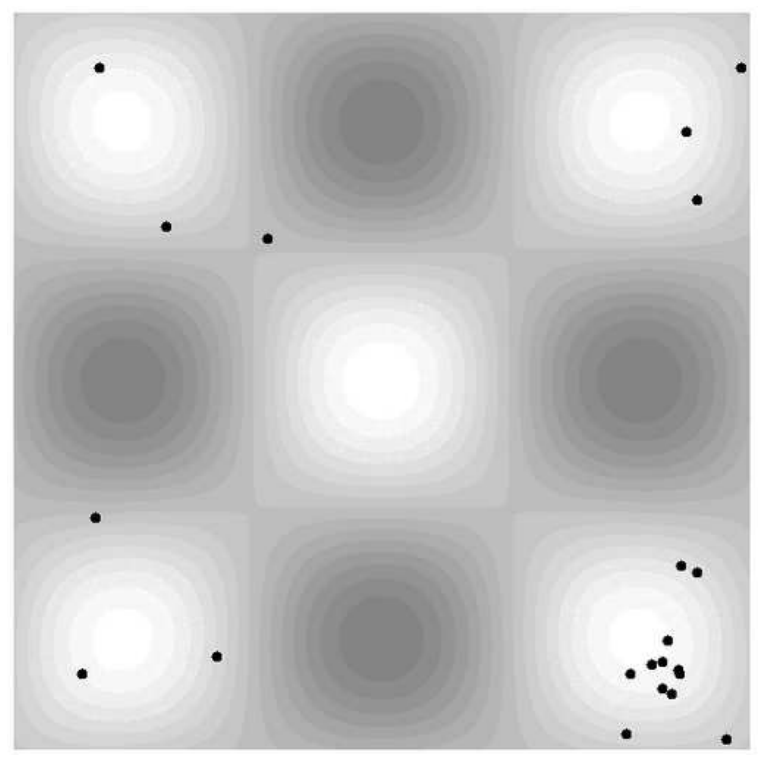

Figure 23a: FN2R45 Initial Chromosome Population before Reproduction.

exploitation within, or converging on, the local minima occupied by the chromosomes $[20]$.

In contrast, in Figure 23c after mutation the chromosomes have dissipated more widely over the solution space. In this sense, mutation is performing its classical function of exploration of the solution space [20]. It is also important to note that it is largely only with mutation that the chromosomes are able to move out of the local optima that they are in and into newer regions of the solution space. This can be seen visually by referring to the bottom right hand corner of Figure 23c where several chromosomes have moved from the local optimum situated there into outer lying regions of the solution space.

The heat maps shown are typical of all those reviewed. The maps showed that while mutation was responsible for exploration of the solution space, crossover was enacting exploitation at the sites of local minima. 


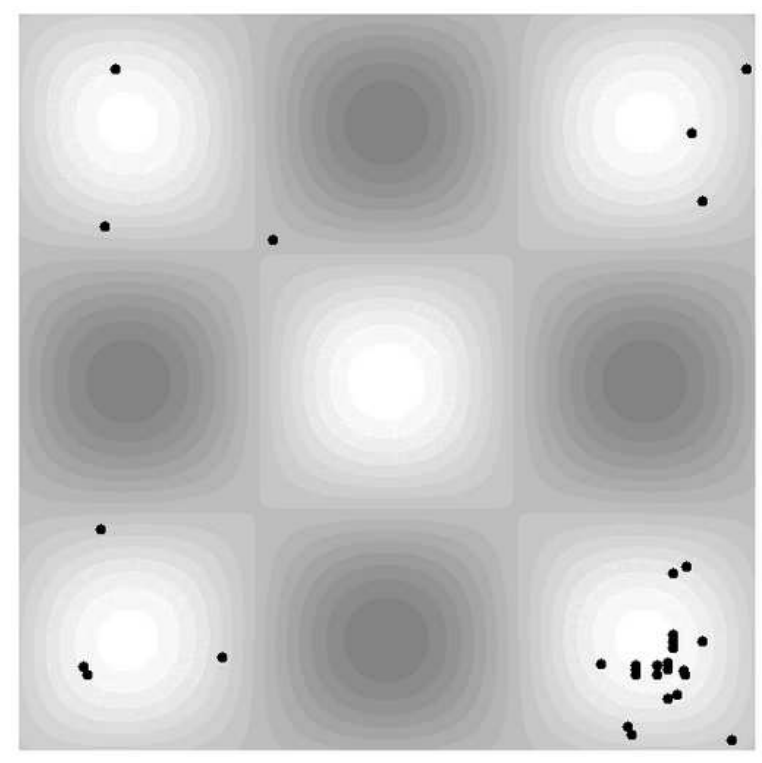

Figure 23b: FN2R45 Chromosome Population after Crossover.

That is, the heat maps showed that crossover was in effect responsible for optimization taking place at the site of local minima thereby keeping chromosomes "stuck" in those local minima. This meant that crossover was having the effect of hindering the movement of chromosomes from local minima into the global minimum.

In order to quantify the degree of optimization at the local minima carried out by crossover the relative proportion of times crossover and mutation improved the best fitness obtained by the population was recorded and compared.

The results were that crossover improved fitness at sites of local minima $82 \%$ of the time out of the total number of epochs (with a $99 \%$ confidence interval of $80 \%$ to $84 \%$ ) compared to mutation with a value of only $30 \%$ (with a confidence interval of $29 \%$ to $31 \%$ ). This lent support to what was visualized on the heat maps, namely, that optimization of chromosomes at local minima due to the crossover operator was hindering chromosomes moving out of these local minima into newer regions of the solution space. 


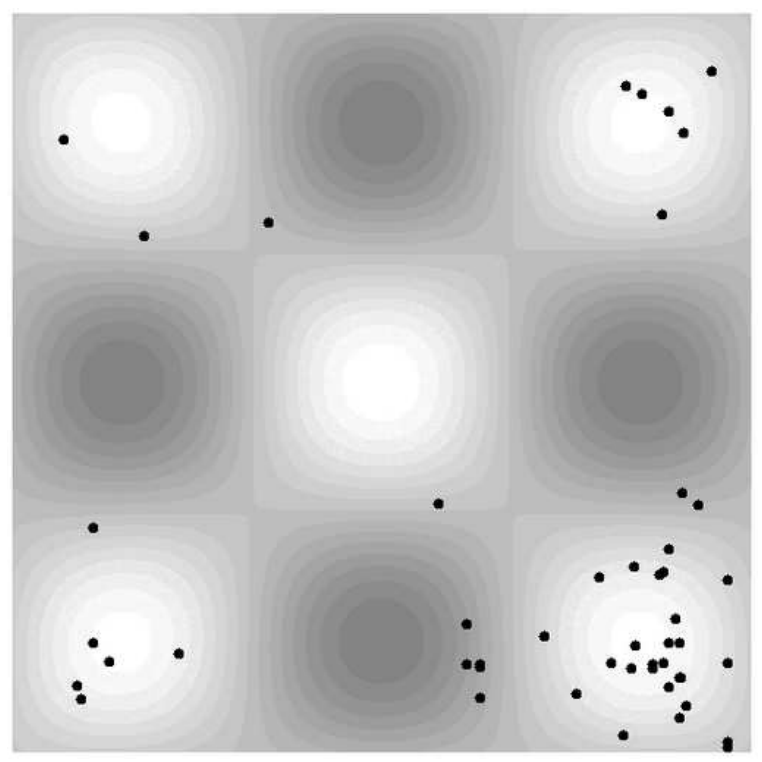

Figure 23c: FN2R45 Chromosome Population after Mutation.

\subsubsection{Bias Associated with the Mutation Operator}

The mutation operator corrupts the reproduction of genotypes thereby introducing the variety that fuels natural selection [4]. This being said, there is discussion in the literature as to the possible biases inherent in various implementations of mutation and the degree to which this makes a problem hard for a GA to solve [3, 4].

Thus, to ascertain in the present work if there was any bias associated with the mutation operator which might make the problems harder for the GA to solve, experiments were carried out where many copies of a single chromosome were mutated and then plotted onto a heat map surface of the rotated function. The chromosome comprised of two bit strings, which were initially placed in the center of the local minimum located in the bottom right hand corner of the heat map of FN2R45. Figure 24 shows an example of this for FN2R45 using the optimal mutation rate of $25.45 \%$ (bit flipping mutation rate of $12.72 \%$ ) with 10000 samples.

As can be seen, after mutation the chromosomes landed in a grid-like pattern along the $x$ and $y$ directions illustrating that it is biased in the axial directions. The reason for this may be explained using a simple example as follows. 


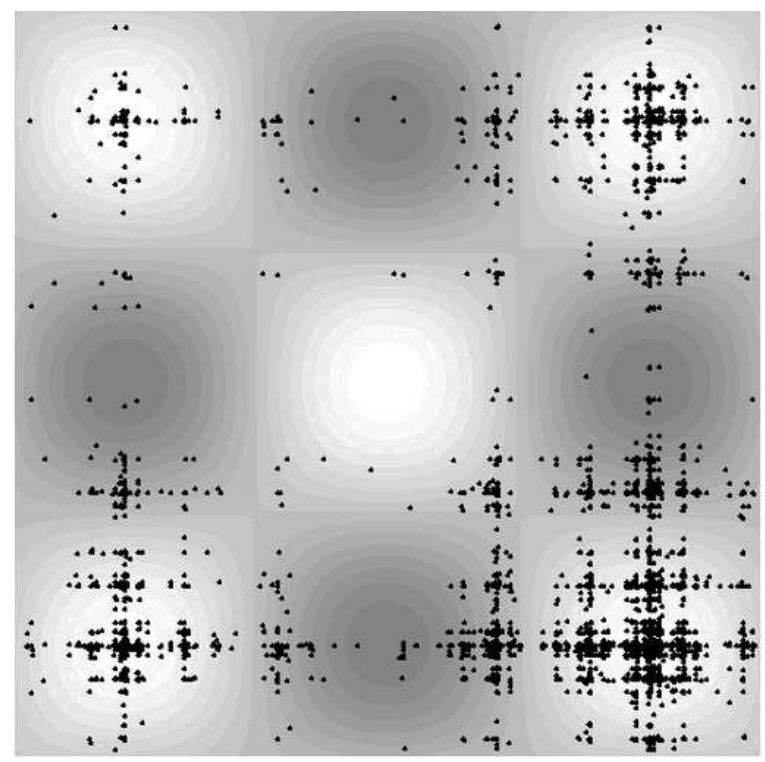

Figure 24: Mutation Plot for Test function FN2R45.

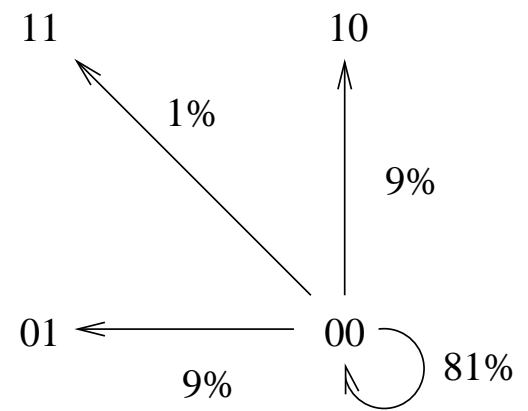

Figure 25: Probabilities associated with the movement of a single two bit chromosome after mutation.

Figure 25 illustrates the probabilities associated with moving in the $x, y$ and diagonal directions for a single two bit chromosome. If we assume that a change in a bit has a probability of $10 \%$, then movement in either the $x$ or $y$ direction has a probability of $9 \%(0.9 \times 0.1)$. By contrast, movement in the diagonal direction requires a change in both bit strings with a resultant probability of $1 \%$. Also, the probability of no change occurring to the chromosome, and hence no movement, is $81 \%$.

Simplistically speaking, for the not-linear-separable problems investigated, the degree to which this bias made the problem hard for the GA was related to the percentage of the local minima which lay on the $x$ and $y$ axes, given that the global 
minimum was at the origin. In Figure 26a for FN2R45 none of the local minima lay on the $x$ or $y$ axes compared with Figure 26b for FN3R45 where 4 of the 12 local minima lay on the $x$ or $y$ axes. Chromosomes in these local minima were more likely to be shifted towards the global minimum due to the bias of the mutation operator. Overviewing the results for all the rotated functions, it was observed that if roughly $20 \%$ or more of the local minima lay along the $x$ or $y$ axes, as shown in Table 18, the crossover operator proved to be beneficial for the function, otherwise it was detrimental.

More generally speaking, this axial bias is a special case of the more general relationship between the problem encoding and the solution space, discussed below.

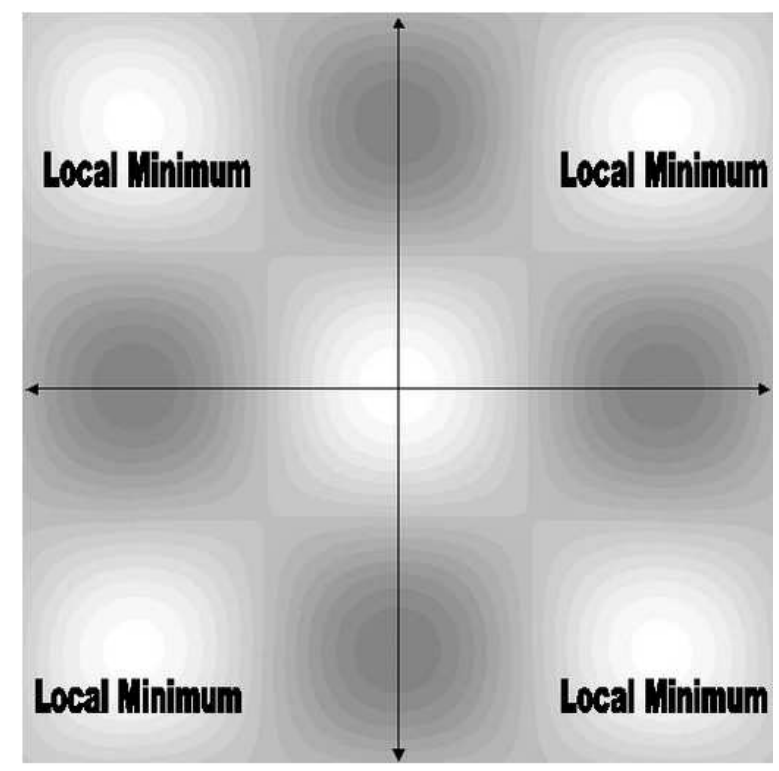

Figure 26a: Heat Map of FN2R45 illustrating location of local minima along $\mathrm{X}$ and $\mathrm{Y}$ axes.

\subsubsection{Relationship between Gray Encoding and the Solu- tion Space}

Figure 24 shows a bias not just in axial directions, but towards a grid-like pattern with regions of higher density and others of much lower density. In general it is much harder to make a "jump" to some areas of the space than others. 


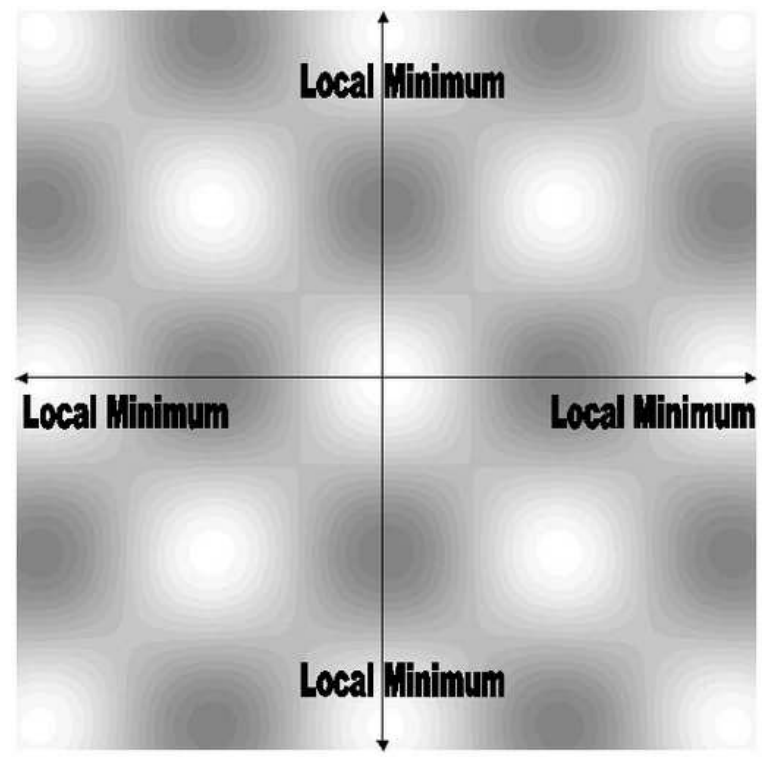

Figure 26b: Heat Map of FN3R45 illustrating location of local minima along $\mathrm{X}$ and $\mathrm{Y}$ axes.

The selection generator compounds the effect of this bias by eliminating candidates that are part way towards a better local minimum but have low fitness.

An illustrative case for the rotated functions is that of FN2R45 and FN3R45. As shown in the response curves depicted in Figure 27a and Figure 27b, FN2R45 was the more difficult of the two functions for the GA. This is evidenced by the fact that the number of epochs taken to reach the threshold was an order of magnitude greater. This is despite the fact that FN3R45 is the more modal of the two functions.

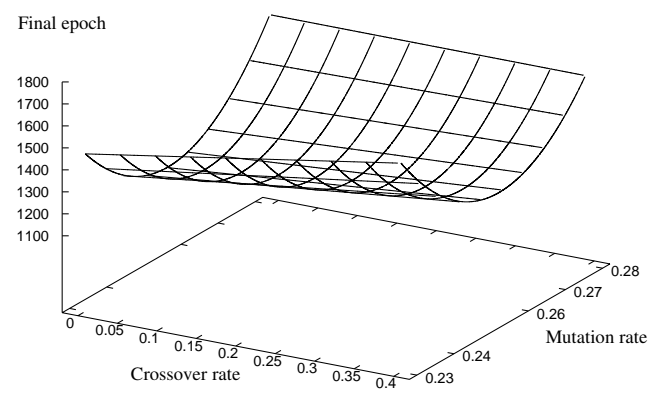

Figure 27a: Response curve for test function FN2R45. 
Table 18: Relationship between Local Minima and Detrimental Crossover

\begin{tabular}{|c|c|c|c|}
\hline $\begin{array}{c}\text { Test } \\
\text { Function }\end{array}$ & $\begin{array}{c}\text { \% Local Minima } \\
\text { on and Y Axes }\end{array}$ & $\begin{array}{c}\text { Detrimental } \\
\text { Crossover }\end{array}$ & $\begin{array}{c}\text { Mean Epochs } \\
\text { to Threshold }\end{array}$ \\
\hline \hline FN1R45 & Nil Local Minima & No & 63.63 \\
\hline FN2R45 & $0 \%$ & Yes & 1381 \\
\hline FN3R45 & $25 \%$ & No & 103.42 \\
\hline FN4R45 & $16.67 \%$ & Yes & 880.0 \\
\hline FN5R45 & $16.67 \%$ & Yes & 727.1 \\
\hline FN6R45 & $20 \%$ & No & 111.1 \\
\hline
\end{tabular}

To illustrate why this is the case, we can examine the Hamming Distances of the two functions. The Hamming Distance is a measure of the difference or distance between two binary sequences of equal length. Hamming Distances between the global minimum and the surrounding local minima for functions FN2R45 and FN3R45 are shown in Figure 28a and Figure 28b, respectively.

As can be seen, FN2R45 has the larger Hamming Distance of 12 from any of the local optima to the global optimum for both the $x$ bit string or the $y$ bit string. The probability of making this (exact) jump with a bit-flipping mutation rate of $m$ for 44-bit chromosomes is:

$$
P_{1}=m^{24}(1-m)^{20}
$$

(Clearly a range of nearby jumps are possible, but we use the minima for illustration. The probability will be higher if nearby jumps are taken into account).

In contrast, for FN3R45, the Hamming Distance from any of the local minima to the global minimum is only 7 or 8 . The probability of making the (exact) jump is therefore of the order:

$$
P_{2}=m^{15}(1-m)^{29}
$$




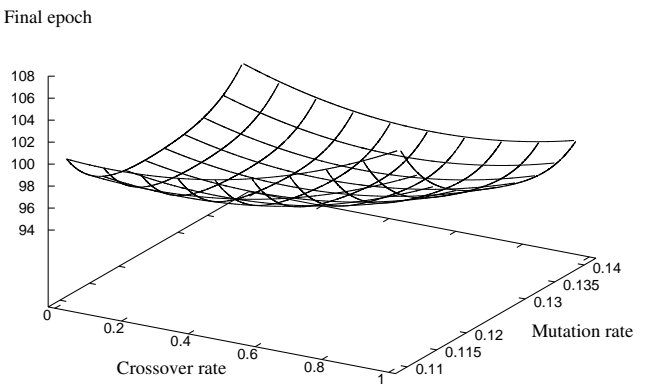

Figure 27b: Response curve for test function FN3R 45.

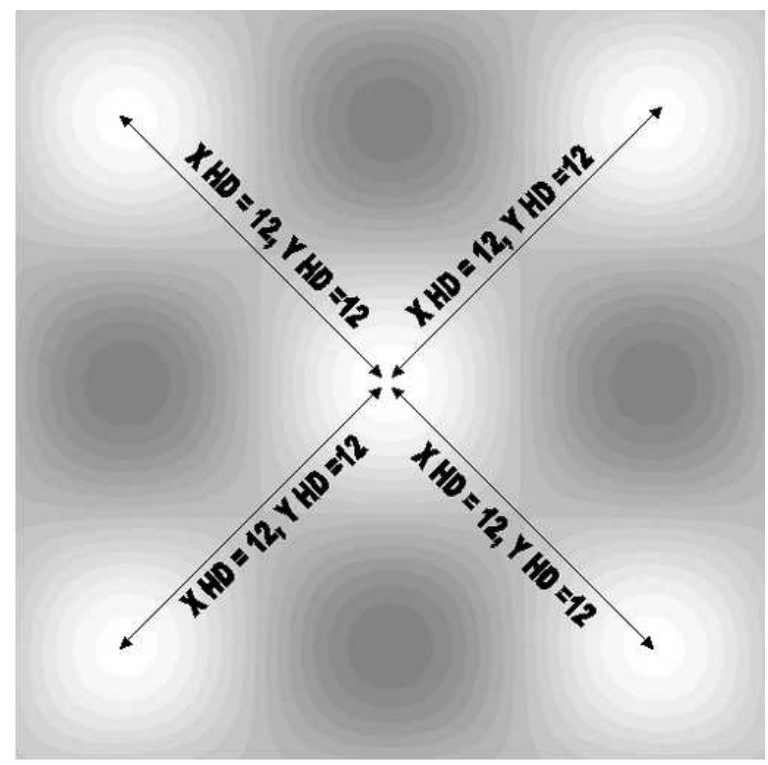

Figure 28a: Hamming Distances for FN2R45.

As can be seen in Figure 29, the probability of making the required jump is far greater for $F N_{3} 45$ for low mutation rates.

The larger Hamming Distances for the functions explained why the optimal mutation rate for FN2R45 was higher (25.45\% corresponding to a bit flipping rate of $12.72 \%)$ than for FN3R45D (12.36\% corresponding to a bit flipping rate of $6.18 \%)$. 


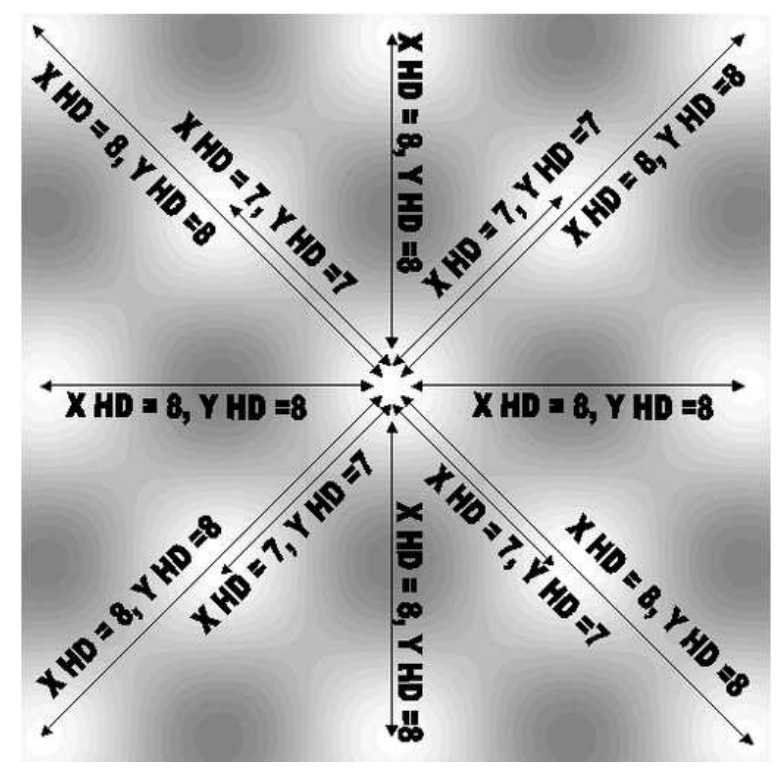

Figure 28b: Hamming Distances for FN3R 45 .

This is because the greater Hamming Distances meant that a greater number of bit flips are required in order to move chromosomes from any of the local optima into the global optimum. These Hamming Distances are a direct consequence of the relationship between the encoding and the solution space.

It is interesting to note that finding the optimal mutation rate appears to be a case of finding a fixed point that is high enough up the Hamming Distance probability curves for the space while at the same time minimizing the disruptive effect of mutation on convergence.

\subsection{Extending the Results to Difficult Practical Problems}

We have discussed a number of properties that make a problem difficult for a GA to solve, such as high modality and local minima not artificially aligned within the encoding to make the solution easier, and their impact on the performance of crossover. However, these have only been tested on artificial sequences of problems 


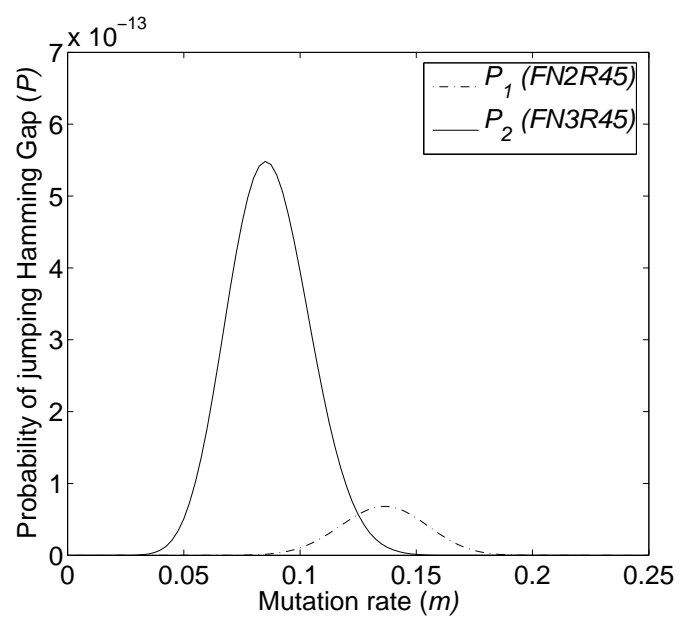

Figure 29: Probability of jumping Hamming Gap versus Mutation rate.

that possess features such as symmetry and a regular repetition of local minima. Before leaving this topic, we wanted to see if there was evidence the results would carry over to real-world problems exhibiting the same properties for difficult problems. In order to extend the results to a difficult practical problem, we tested our GA on Landscape 20_101 shown in Figure 22. The results are illustrated in Table C-4, Table C-6 and Table C-7.

The same behaviour of the GA emerged as for the difficult rotated functions. That is, crossover, mutation and their interaction had a statistically significant effect upon GA performance. However, for crossover the effect was detrimental with an optimal crossover rate of $0 \%$.

For mutation the optimal rate was a high $18.93 \%$ (bit flipping mutation rate of $9.46 \%$ ), comparable to the high mutation rates seen with the difficult not-linearseparable problems discussed above. Again as noted above, a high mutation rate is a conjectured marker for the difficulty of the problem.

It can be conjectured that this problem proved difficult for the GA for similar reasons to the problems analyzed earlier. In the first case the random arrangement of the local minima of this problem makes it unlikely that any of the local minima are aligned in the axial directions. Thus, the bias of mutation means that it is less 
likely that the global minimum will be found by chromosomes moving in the $x$ and $y$ directions.

In reference to crossover, the fact that the surface of the Landscape 20_101 has a great number of local minima means that it is very likely that crossover was enacting optimization at the local minima sites. This is supported by the fact that the optimal mutation rate was high at $18.93 \%$ (bit flipping mutation rate of $9.46 \%$ ), suggesting that a high mutation rate was required to get chromosomes to jump out of regions of local minima where they were "stuck" due to local optimization carried out by crossover.

\subsection{Discussion}

The traditional concept of a GA, that of selection, crossover and mutation, is being challenged as literature has emerged which suggests that the crossover operator may not necessarily be essential in a GA. However, there has not as yet been a direct statistical attempt to prove the detrimentality of crossover nor an attempt to describe the conditions under which such detrimentality may occur. This chapter used our statistical methodology to explore the issue of the detrimentality of crossover. In particular, we were interested in establishing whether not-linear-separability was a sufficient determinant of the detrimentality of crossover and if not, what other factors was it characterized by.

In the first instance the results from the linear-separable test function series, FNn, show that crossover is beneficial for these linear-separable problems. This concurs with the suggestion of Salomon that Crossover's Niche is in fact linear-separable problems [38].

On the other hand, results from the rotated not-linear-separable test function series demonstrated several instances where crossover was statistically proven to be detrimental. This occurred for not-linear-separable problems which required the highest mutation rates, which has been a marker for the difficulty of a problem. Thus, what 
makes a not-linear-separable problem hard for a GA to solve is linked to whether crossover will be detrimental to the performance of the GA solving the problem.

In the course of the present research it was found that three factors were involved in making a not-linear-separable problem hard for the GA to solve. These were optimization carried out by crossover at the sites of local minima, the bias of the mutation operator and the Hamming Distances for the individual problems.

In the first case, the difficulty of a problem was impacted by the degree of optimization at local minima carried out by the crossover operator. That is, crossover was carrying out optimization on chromosomes "stuck" in local minima resulting in their moving deeper into the local minima sites. Our experiments on this showed that at least $80 \%$ of the time crossover improved the fitness of chromosomes at sites of local minima.

Secondly, it was found that the mutation operator was biased along the $x$ and $y$ axes. If a function had at least some of the local minima and the global minimum aligned in the axial directions this made the problem easier to solve as the chromosomes from these minima would be shifted with a greater likelihood towards the global minimum.

Thirdly, the relationship between the problem and the solution space resulted in situations where a less modal problem was actually more difficult to solve because of the greater Hamming Distance between its local minima and the global minimum. This was illustrated for FN2R45 and FN3R 45 where the latter was the more modal function, yet proved easier to solve as the Hamming Distances between its local minima and its global minimum were lower.

Finally, the detrimentality of crossover was demonstrated on a difficult practical problem, namely, a problem from the Huygens suite. The results showed that crossover can be detrimental on a real world problem. The reasons for this occurring may be extrapolated from the reasons found for the difficult rotated $F N n$ problem series. These include the degree of local optimization attributable to the crossover 
operator and the bias of the mutation operator.

In conclusion, it has been demonstrated that crossover is statistically detrimental for the difficult not-linear-separable problems and also the difficult real world problem in the given configuration. Further research will be required to extend the class of problems and illustrate if crossover can be demonstrated to be detrimental with different encodings and in discrete problem domains. However, the results suggest that crossover can prove to have a truly detrimental effect upon GA performance. It should be noted that the results apply to specific (one-point) crossover operator and mutation operator and further tests would be required to determine whether other crossover operators (such as uniform crossover) and mutation give similar results. 


\section{Chapter 6}

\section{General Conclusions and Future}

\section{Research}

G enetic algorithms have been the focus of extensive study in computer science and have been applied to both theoretical and real world problems. However, there has been no generally accepted methodology to assess which parameters significantly affect performance of genetic algorithms, whether these parameters interact and how performance varies with respect to changes in parameters. The focus of this thesis has been to formulate a statistical methodology for the exploratory study of genetic and other adaptive algorithms and to demonstrate the application of the methodology through the investigation of properties of a GA.

\subsection{Statistical Methodology}

The first part of the present thesis dealt with the development of a statistical methodology for the exploratory analysis of genetic and other adaptive algorithms. To recap, once the algorithm and the problem domain have been specified, the steps in the statistical methodology proceed as follows:

1. Identify sources of variation and modify the algorithm to generate blocked 
runs.

2. Use a workup procedure to minimize the appearance of censored observations and to finalize starting ranges for parameters.

3. Generate an initial data-set consisting of an arbitrary number of replicates. Typically, we have found 100 replicates to be a useful starting point.

4. Calculate power based upon a chosen effect size. We recommend an effect size index of 0.4 (large effect). If at least $80 \%$ power is not achieved and the experiment resulted in observing no interaction increase the sample size.

5. Conduct (pooled) ANOVA analysis and determine which parameters are statistically significant.

6. For parameters which are statistically significant partition the sum of squares into polynomial contrast terms. Determine which polynomial terms are statistically significant.

7. Use polynomial regression to obtain the coefficients for the overall response curve (if the interaction parameter is statistically significant) or to obtain the coefficients for the response curve for each parameter separately (if the interaction parameter is not statistically significant).

8. Differentiate and solve the response curve for each parameter to obtain best values and calculate confidence intervals.

The statistical methodology developed was initially trialed on well known test functions. Looking at the results from the suite of test functions together, we found that crossover appears to have a predominantly linear effect and that the direction of its slope is problem specific. In contrast, mutation appears to have a predominantly quadratic effect upon performance. The mutation rates observed advocate the use of higher mutation rates than have traditionally been used. The use of statistics 
also enabled the issue of interaction to be addressed and we found that whether interaction is significant is also problem specific.

These initial trials enabled the identification of key features affecting GA performance that deserved more detailed investigation. Our subsequent work demonstrated how the statistical methodology can assist in guiding the GA practitioner to explore such features.

\subsection{The Importance of Interaction}

The second part of this thesis examined the issue of whether crossover and mutation interact or if each parameter exerts its effect independently. This led to two important questions. First, what type of functions are likely to demonstrate interaction between crossover and mutation, and, secondly, what is the practical implication of interaction when attempting to obtain optimal rates for these parameters. These questions were addressed by examining the relationship between the occurrence of statistically significant interaction among crossover and mutation and increasing modality of a problem.

Addressing the first question it was found that functions with increased modality are more likely to demonstrate interaction between crossover and mutation. It is conjectured that when dealing with highly modal functions the possibility of interaction must be considered. For simple functions, with little or no multi-modality, it is conjectured that crossover and mutation are exerting their respective effects independently.

Addressing the second question it has been shown that if interaction is occurring attempting to optimize the rate of crossover and mutation independently may result in rates which are not optimal. In order to account for the effect of interaction all combinations of crossover and mutation, within given starting ranges, must be trialed. 


\subsection{The Influence of Gray Encoding}

The third part of this thesis explored which factors may affect GA performance. This led to two important questions, namely, whether there is a statistically significant relationship between the difficulty of a problem and the choice of encoding, and, if so, what is the actual mechanism by which this occurs.

In addressing the first question, this chapter demonstrated that a lower modality problem may be significantly more difficult to solve with a Gray encoding than a higher modality problem. This contrasts with the usual trend of problem difficulty increasing with increasing modality.

In addressing the second question, animations of the GA clearly showed the ability of chromosomes to move between local optima and avoid culling in the functions studied. The probability of a successful jump is dependent on the Hamming Distance. Calculation was therefore made of the Hamming Distances between local optima present in the two functions and it was found that movement within the lower modality function was more difficult because of the significantly higher Hamming Distances involved. These Hamming Distances are a direct result of the encoding. In conclusion, it has been demonstrated that there is a real relationship between the difficulty of a problem and the choice of encoding, in this instance Gray codes. It has further been conjectured that the mechanism by which this occurs is related to the different Hamming Distances occurring at specific regions in the solution space.

\subsection{The Detrimentality of Crossover}

In the first part of the present thesis an interesting observation was that the optimal crossover rate for De Jong's F2 and Schaffer's F6 was $0 \%$ in our experimental set-up. This implied that crossover was acting detrimentally on these occasions. A limited amount of work has conjectured that the niche for the beneficial effect of crossover upon GA performance is related to linear-separability, and this was borne in these 
initial test functions. To explore this relationship in more detail, we compared two problem suites, one of which was linear-separable and the other not-linear-separable (the latter functions having been rotated by 45 degrees in the solution space).

Rather, we found that not-linear-separability was not, on its own, a sufficient determinant for the detrimentality of crossover. It was shown that the crossover operator was detrimental to the performance of the GA for difficult rotated functions. It is conjectured that what makes a problem difficult for the GA involves factors such as the degree of optimization at local minima due to crossover, the bias associated with the mutation operator and the Hamming Distances present in the individual problems due to the encoding.

Finally, the GA was tested on a real world landscape minimization problem to ascertain if the results obtained would match those associated with the difficult rotated functions. It was shown that they match and that the features which make certain of the test functions difficult are also present in the real world problem.

\subsection{Future Research}

This thesis has demonstrated a statistical methodology that allows the investigator to undertake exploratory analysis of genetic and other adaptive algorithms. This methodology has then been used to explore the issue of interaction between crossover and mutation, the influence of the encoding used (in this thesis Gray encoding) and the detrimentality of crossover. Given the unique advantages offered by statistical analysis, such as the ability to block for seed, calculation of power and sample size, and rigorous study of response curves, further use of statistics will assist in the development of GAs as powerful search tools.

This being said, there are a number of limitations in the present thesis which warrant future research. In the first instance, the implementation of the GA was deliberately simple so that a clear and concise demonstration of the proposed methodology and results could be made. In this regard parameters such as the population size 
and bits per variable were not varied and only crossover and mutation were investigated in the present thesis. The methodology described in this thesis can be straightforwardly applied to the many other parameters suggested in the literature by including these as extra parameters.

Secondly, the functions examined in this thesis have been continuous functions. There are, however, many other problem types to which this methodology may be applied. Examples include constrained optimization problems, multi-objective optimization problems and discrete combinatorial optimization problems. Application of the statistical methodology presented in this thesis to these problem domains and others would provide a greater understanding of the performance of genetic algorithms.

Finally, this thesis has concerned itself solely with GAs. The methodology however can be applied to other adaptive algorithms such as Particle Swarm Optimization (PSO) and Differential Evolution in a similar fashion to that applied to GAs. Research in this field would greatly increase our understanding about the comparative performance of different types of adaptive algorithms and their sensitivity to the parameters on which they are based. 


\section{Bibliography}

[1] T Bäck, D Fogel and Z Michalewicz (editors). Handbook of Evolutionary Computation. Oxford University Press, 1997.

[2] T Bäck, U Hammel and H Schwefel. Evolutionary computation: comments on the history and current state. IEEE Transactions on Evolutionary Computation, Volume 1, Number 1, April 1997.

[3] S Bullock. Are artificial mutation biases unnatural? In European Conference on Artificial Life, pages 64-73. Springer, 1999.

[4] S Bullock. Smooth operator? Understanding and visualising mutation bias. Lecture Notes in Computer Science, Volume 2159, pages 602-612, Springer, 2001.

[5] J Cohen. Statistical Power Analysis for the Behavioral Sciences. Lawrence Erlbaum, 1988.

[6] L Davis (editor). Handbook of genetic algorithms. Van Nostrand Reinhold, 1991.

[7] L Davis. Adapting operator probabilities in genetic algorithms. In Proc. Third International Conference on Genetic Algorithms, pages 61-69, Morgan Kaufmann, 1989. 
[8] T E Davis and J C Principe. A markov chain framework for the simple genetic algorithm. Evolutionary Computation, Volume 1, Number 3, pages 269-288, 1993.

[9] K A De Jong. Analysis of the Behavior of a Class of Genetic Adaptive Systems. Phd dissertation, Department of Computer and Communication Sciences, University of Michigan, Ann Arbor, MI, 1975.

[10] S Droste, T Jansen and I Wegener. On the analysis of the $(1+1)$ evolutionary algorithm. Theoretical Computer Science, Volume 276, pages 51-81, 2002.

[11] A E Eiben, R Hinterding and Z Michalewicz. Parameter control in evolutionary algorithms. IEEE Transactions on Evolutionary Computation, Volume 3, Number 2, pages 124-141, 1999.

[12] L Eshelman. Bit-climbers and naive evolution. Genetic Algorithms Digest, Volume 5, Number 39, December 1991.

[13] L J Eshelman and J D Schaffer. Crossover's niche. In Proc. Fifth International Conference on Genetic Algorithms, pages 9-14, Morgan Kaufmann, 1993.

[14] F Faul and E Erdfelder. GPOWER: A priori, post-hoc, and compromise power analyses computer program for MS-DOS. Available: http://www.psycho.uniduesseldorf.de/aap/projects/gpower/index.html. Bonn, FRG: Bonn University, Dep. of Psychology.

[15] D B Fogel and J W Atmar. Comparing genetic operators with gaussian mutations in simulated evolutionary processes using linear systems. Biological Cybernetics, Volume 63, Number 2, pages 111-114, 1990.

[16] B Freisleben and M Härtfelder. Optimization of genetic algorithms by genetic algorithms. In Proc. International Conference on Aritifical Neural Nets and Genetic Algorithms, pages 392-399. Springer-Verlag, 1993. 
[17] D E Goldberg. Genetic algorithms in search, optimization and machine learning. Addison-Wesley, 1989.

[18] J Grefenstette. Optimization of control parameters for genetic algorithms. IEEE Transactions on Systems, Man, and Cybernetics, Volume SMC-16, Number $1,1986$.

[19] J He and X Yao. From an individual to a population: an analysis of the first hitting time of population-based evolutionary algorithms. IEEE Transactions on Evolutionary Computation, Volume 6, Number 5, pages 495-511, 2002.

[20] J H Holland. Adaptation in natural and artificial systems. University of Michigan Press, 1975.

[21] Insightful. S-PLUS. Available: http://www.insightful.com/.

[22] T Jansen and I Wegener. The analysis of evolutionary algorithms - A proof that crossover really can help. Algorithmica, Volume 34, pages 47-66, 2002.

[23] T Jansen and I Wegener. Real royal road functions-where crossover provably is essential. Discrete Applied Mathematics, Volume 149, pages 111-125, 2005.

[24] R A Johnson and G K Bhattacharyya. Statistics: Principles and Methods. John Wiley \& Sons, 1996.

[25] T Jones. Crossover, macromutation, and population-based search. In Proc. of the Sixth International Conference on Genetic Algorithms, pages 73-80, Morgan Kaufman, 1995.

[26] R I Levin and D S Rubin. Applied Elementary Statistics. Prentice-Hall, 1980.

[27] C MacNish. Towards unbiased benchmarking of evolutionary and hybrid algorithms for real-valued optimisation. To appear in Connection Science, special issue on Evolutionary Learning and Optimization. 
[28] C MacNish. Benchmarking evolutionary and hybrid algorithms using randomized self-similar landscapes. In Proc. Sixth International Conference on Simulated Evolution and Learning SEAL, Volume 4247, pages 361-368. Springer, 2006.

[29] M Mitchell, S Forrest and J Holland. The royal road for genetic algorithms: Fitness landscapes and GA performance. In Towards a Practice of Autonomous Systems: Proc. of the First European Conference on Artificial Life, pages 245254. MIT Press, 1991.

[30] D C Montgomery. Design and Analysis of Experiments. John Wiley and Sons, 1976.

[31] A Narayanan and M Moore. Quantum inspired genetic algorithms. In International Conference on Evolutionary Computation, pages 61-66. IEEE, 1996.

[32] A Petrovski and J McCall. Optimising GA parameters using statistical approaches. Technical report, Robert Gordon University, 1997.

[33] A Petrovski, A Wilson and J McCall. Statistical analysis of genetic algorithms and inference about optimal factors. Technical report, Robert Gordon University, 1998.

[34] A Prugel-Bennett and J L Shapiro. Analysis of genetic algorithms using statistical mechanics. Physical Review Letters, Volume 72, Number 9, pages 13051309, 1994.

[35] C R Reeves and C C Wright. Genetic algorithms and the design of experiments. In Evolutionary Algorithms: IMA Volumes in Mathematics and its Applications, Volume 111, pages 207-226. Springer-Verlag, New York, 1999.

[36] J A Rice. Mathematical Statistics and Data Analysis. Duxbury Press, 1995.

[37] F Rothlauf. Representations for Genetic and Evolutionary Algorithms. Springer, 2003. 
[38] R Salomon. Re-evaluating genetic algorithm performance under coordinate rotation of benchmark functions: a survey of some theoretical and practical aspects of genetic algorithms. BioSystems, Volume 39, Number 3, pages 263$278,1996$.

[39] J D Schaffer, R A Caruana, L J Eshelman and R Das. A study of control parameters affecting online performance of genetic algorithms for function optimization. In Proc. Third International Conference on Genetic algorithms, pages 51-60. Morgan Kaufmann, 1989.

[40] A B Simões and E Costa. Transposition versus crossover: an empirical study. In Proc. Genetic and Evolutionary Computation Conference GECCO, pages 612-619. Morgan Kaufmann, 1999.

[41] Sun Microsystems, Inc. Java version 1.3.1_02.

[42] D H Wolpert and W G Macready. No free lunch theorems for optimization. IEEE Transactions on Evolutionary Computation, Volume 1, Number 1, pages 67-82, 1997.

[43] X Yao, Y Liu and G Lin. Evolutionary programming made faster. IEEE Transactions on Evolutionary Computation, Volume 3, Number 2, pages 82102, 1999. 


\section{Appendix A}

\section{F1, F3, F2 and F6}

\section{A Results}

\section{ANOVA Tables}

Table A-1: F1-Power with 100 replicates

\begin{tabular}{|c|c|c|c|}
\hline Parameter & Difference (epochs) & Effect size index $f$ & Power \\
\hline \hline Crossover & 10 & 0.17154 & $100 \%$ \\
\hline Crossover & 5 & 0.08578 & $99.99 \%$ \\
\hline Crossover & 3 & 0.05146 & $84.11 \%$ \\
\hline Crossover & 2 & 0.03431 & $35.36 \%$ \\
\hline Crossover & 1 & 0.01715 & $5.19 \%$ \\
\hline Crossover & Large & 0.4 & $100 \%$ \\
\hline Crossover & Medium & 0.25 & $100 \%$ \\
\hline Crossover & Small & 0.1 & $100 \%$ \\
\hline \hline Mutation & 10 & 0.13684 & $100 \%$ \\
\hline Mutation & 5 & 0.06842 & $97.84 \%$ \\
\hline Mutation & 3 & 0.04105 & $44.53 \%$ \\
\hline Mutation & 2 & 0.02737 & $13.03 \%$ \\
\hline Mutation & 1 & 0.01368 & $2.57 \%$ \\
\hline Mutation & Large & 0.4 & $100 \%$ \\
\hline Mutation & Medium & 0.25 & $100 \%$ \\
\hline Mutation & Small & 0.1 & $100 \%$ \\
\hline
\end{tabular}


Table A-2: F1-Power with 100 replicates continued

\begin{tabular}{|l|c|c|c|}
\hline Parameter & Difference (epochs) & Effect size index $f$ & Power \\
\hline \hline Interaction & 10 & 0.05172 & $27.58 \%$ \\
\hline Interaction & 5 & 0.02586 & $\mathbf{3 . 3 8 \%}$ \\
\hline Interaction & 3 & 0.01552 & $1.62 \%$ \\
\hline Interaction & 2 & 0.01034 & $1.25 \%$ \\
\hline Interaction & 1 & 0.00517 & $1.06 \%$ \\
\hline Interaction & Large & 0.4 & $100 \%$ \\
\hline Interaction & Medium & 0.25 & $100 \%$ \\
\hline Interaction & Small & 0.1 & $99.52 \%$ \\
\hline
\end{tabular}

Mean square error $=15.58005$ epochs. 
Table A-3: F1-Power with 500 replicates

\begin{tabular}{|c|c|c|c|}
\hline Parameter & Difference (epochs) & Effect size index $f$ & Power \\
\hline \hline Crossover & 10 & 0.17041 & $100 \%$ \\
\hline Crossover & 5 & 0.08520 & $100 \%$ \\
\hline Crossover & 3 & 0.05112 & $100 \%$ \\
\hline Crossover & 2 & 0.03408 & $>99.37 \%$ \\
\hline Crossover & 1 & 0.01704 & $>36.65 \%$ \\
\hline Crossover & Large & 0.4 & $100 \%$ \\
\hline Crossover & Medium & 0.25 & $100 \%$ \\
\hline Crossover & Small & 0.1 & $100 \%$ \\
\hline \hline Mutation & 10 & 0.13594 & $100 \%$ \\
\hline Mutation & 5 & 0.06797 & $100 \%$ \\
\hline Mutation & 3 & 0.04078 & $>99.94 \%$ \\
\hline Mutation & 2 & 0.02719 & $>83.66 \%$ \\
\hline Mutation & 1 & 0.01359 & $>13.55 \%$ \\
\hline Mutation & Large & 0.4 & $100 \%$ \\
\hline Mutation & Medium & 0.25 & $100 \%$ \\
\hline Mutation & Small & 0.1 & $100 \%$ \\
\hline \hline Interaction & 10 & 0.05138 & $>99.84 \%$ \\
\hline Interaction & 5 & 0.02569 & $>\mathbf{2 9 . 0 6 \%}$ \\
\hline Interaction & 3 & 0.01541 & $>5.40 \%$ \\
\hline Interaction & 2 & 0.01028 & $>2.33 \%$ \\
\hline Interaction & 1 & 0.00514 & $>1.26 \%$ \\
\hline Interaction & Large & 0.4 & $100 \%$ \\
\hline Interaction & Medium & 0.25 & $100 \%$ \\
\hline Interaction & Small & 0.1 & $100 \%$ \\
\hline
\end{tabular}

Mean square error $=15.68375$ epochs.

Note: GPOWER can only accept sample sizes of up to 32000.

The sample size for 500 replicates was 38500 .

Thus, where a $>$ symbol is used power was calculated using a sample size of 32000 while the actual power would be marginally greater. 
Table A-4: F1-Power of the pooled analysis

\begin{tabular}{|c|c|c|c|}
\hline Parameter & Difference (epochs) & Effect size index $f$ & Power \\
\hline \hline Crossover & 10 & 3.9193 & $100 \%$ \\
\hline Crossover & 5 & 1.9597 & $100 \%$ \\
\hline Crossover & 3 & 1.1758 & $100 \%$ \\
\hline Crossover & 2 & 0.78386 & $100 \%$ \\
\hline Crossover & 1 & 0.39193 & $100 \%$ \\
\hline Crossover & Large & 0.4 & $100 \%$ \\
\hline Crossover & Medium & 0.25 & $90.39 \%$ \\
\hline Crossover & Small & 0.1 & $9.83 \%$ \\
\hline \hline Mutation & 10 & 3.1265 & $100 \%$ \\
\hline Mutation & 5 & 1.5633 & $100 \%$ \\
\hline Mutation & 3 & 0.93796 & $100 \%$ \\
\hline Mutation & 2 & 0.62531 & $100 \%$ \\
\hline Mutation & 1 & 0.31265 & $97.94 \%$ \\
\hline Mutation & Large & 0.4 & $99.99 \%$ \\
\hline Mutation & Medium & 0.25 & $82.55 \%$ \\
\hline Mutation & Small & 0.1 & $6.96 \%$ \\
\hline \hline Interaction & 10 & 1.1817 & $100 \%$ \\
\hline Interaction & 5 & 0.59086 & $\mathbf{1 0 0 \%}$ \\
\hline Interaction & 3 & 0.35452 & $79.01 \%$ \\
\hline Interaction & 2 & 0.23634 & $23.79 \%$ \\
\hline Interaction & 1 & 0.11817 & $3.11 \%$ \\
\hline Interaction & Large & 0.4 & $92.65 \%$ \\
\hline Interaction & Medium & 0.25 & $29.05 \%$ \\
\hline Interaction & Small & 0.1 & $2.33 \%$ \\
\hline
\end{tabular}

Mean square error $=0.6819076$ epochs. 
Table A-5: F3-Power of the pooled analysis

\begin{tabular}{|c|c|c|c|}
\hline Parameter & Difference (epochs) & Effect size index $f$ & Power \\
\hline \hline Crossover & 10 & 2.6652 & $100 \%$ \\
\hline Crossover & 5 & 1.3326 & $100 \%$ \\
\hline Crossover & 3 & 0.79956 & $100 \%$ \\
\hline Crossover & 2 & 0.53304 & $100 \%$ \\
\hline Crossover & 1 & 0.26652 & $75.25 \%$ \\
\hline Crossover & Large & 0.4 & $99.49 \%$ \\
\hline Crossover & Medium & 0.25 & $67.45 \%$ \\
\hline Crossover & Small & 0.1 & $6.26 \%$ \\
\hline \hline Mutation & 10 & 1.9865 & $100 \%$ \\
\hline Mutation & 5 & 0.99327 & $100 \%$ \\
\hline Mutation & 3 & 0.59596 & $100 \%$ \\
\hline Mutation & 2 & 0.39731 & $97.74 \%$ \\
\hline Mutation & 1 & 0.19865 & $26.92 \%$ \\
\hline Mutation & Large & 0.40 & $97.93 \%$ \\
\hline Mutation & Medium & 0.25 & $51.41 \%$ \\
\hline Mutation & Small & 0.1 & $4.12 \%$ \\
\hline \hline Interaction & 10 & 0.88840 & $100 \%$ \\
\hline Interaction & 5 & 0.44420 & $\mathbf{8 8 . 2 7 \%}$ \\
\hline Interaction & 3 & 0.26652 & $23.21 \%$ \\
\hline Interaction & 2 & 0.17768 & $6.34 \%$ \\
\hline Interaction & 1 & 0.08884 & $1.76 \%$ \\
\hline Interaction & Large & 0.4 & $75.30 \%$ \\
\hline Interaction & Medium & 0.25 & $18.64 \%$ \\
\hline Interaction & Small & 0.1 & $2.02 \%$ \\
\hline
\end{tabular}

Mean square error $=1.1865$ epochs . 
Table A-6: F2-Power of the pooled analysis

\begin{tabular}{|c|c|c|c|}
\hline Parameter & Difference (epochs) & Effect size index $f$ & Power \\
\hline \hline Crossover & 10 & 0.27104 & $100 \%$ \\
\hline Crossover & 5 & 0.13552 & $56.28 \%$ \\
\hline Crossover & 3 & 0.08131 & $11.87 \%$ \\
\hline Crossover & 2 & 0.05421 & $4.05 \%$ \\
\hline Crossover & 1 & 0.02710 & $1.53 \%$ \\
\hline Crossover & Large & 0.4 & $100 \%$ \\
\hline Crossover & Medium & 0.25 & $99.96 \%$ \\
\hline Crossover & Small & 0.1 & $22.88 \%$ \\
\hline \hline Mutation & 10 & 0.29113 & $100 \%$ \\
\hline Mutation & 5 & 0.14557 & $70.38 \%$ \\
\hline Mutation & 3 & 0.08734 & $16.61 \%$ \\
\hline Mutation & 2 & 0.05823 & $5.24 \%$ \\
\hline Mutation & 1 & 0.02911 & $1.69 \%$ \\
\hline Mutation & Large & 0.40 & $100 \%$ \\
\hline Mutation & Medium & 0.25 & $99.98 \%$ \\
\hline Mutation & Small & 0.1 & $25.48 \%$ \\
\hline \hline Interaction & 10 & 0.07517 & $2.04 \%$ \\
\hline Interaction & 5 & 0.03759 & $\mathbf{1 . 2 1 \%}$ \\
\hline Interaction & 3 & 0.02255 & $1.07 \%$ \\
\hline Interaction & 2 & 0.01503 & $1.03 \%$ \\
\hline Interaction & 1 & 0.00752 & $1.01 \%$ \\
\hline Interaction & Large & 0.4 & $99.97 \%$ \\
\hline Interaction & Medium & 0.25 & $62.57 \%$ \\
\hline Interaction & Small & 0.1 & $3.32 \%$ \\
\hline
\end{tabular}

Mean square error $=6.736177$ epochs. 
Table A-7: F6-Power of the pooled analysis

\begin{tabular}{|c|c|c|c|}
\hline Parameter & Difference (epochs) & Effect size index $f$ & Power \\
\hline \hline Crossover & 10 & .28308 & $100 \%$ \\
\hline Crossover & 5 & .14154 & $72.65 \%$ \\
\hline Crossover & 3 & .08492 & $17.11 \%$ \\
\hline Crossover & 2 & .05661 & $5.30 \%$ \\
\hline Crossover & 1 & .02830 & $1.69 \%$ \\
\hline Crossover & Large & .4 & $100 \%$ \\
\hline Crossover & Medium & .25 & $99.99 \%$ \\
\hline Crossover & Small & .1 & $28.86 \%$ \\
\hline \hline Mutation & 10 & .28308 & $100 \%$ \\
\hline Mutation & 5 & .14154 & $72.65 \%$ \\
\hline Mutation & 3 & .08492 & $17.11 \%$ \\
\hline Mutation & 2 & .05661 & $5.30 \%$ \\
\hline Mutation & 1 & .02830 & $1.69 \%$ \\
\hline Mutation & Large & .4 & $100 \%$ \\
\hline Mutation & Medium & .25 & $99.99 \%$ \\
\hline Mutation & Small & .1 & $28.86 \%$ \\
\hline \hline Interaction & 10 & .07309 & $2.05 \%$ \\
\hline Interaction & 5 & .03654 & $\mathbf{1 . 2 1 \%}$ \\
\hline Interaction & 3 & .02192 & $1.07 \%$ \\
\hline Interaction & 2 & .01461 & $1.03 \%$ \\
\hline Interaction & 1 & .00730 & $1.01 \%$ \\
\hline Interaction & Large & 0.4 & $99.99 \%$ \\
\hline Interaction & Medium & 0.25 & $69.01 \%$ \\
\hline Interaction & Small & 0.1 & $3.56 \%$ \\
\hline
\end{tabular}

Mean square error $=6.449417$ epochs. 
Table A-8: F6-Power of the pooled analysis for crossover 0\% to $15 \%$

\begin{tabular}{|c|c|c|c|}
\hline Parameter & Difference (epochs) & Effect size index $f$ & Power \\
\hline \hline Crossover & 10 & .32905 & $100 \%$ \\
\hline Crossover & 5 & .16452 & $\mathbf{9 1 . 6 3 \%}$ \\
\hline Crossover & 3 & .09871 & $29.32 \%$ \\
\hline Crossover & 2 & .06581 & $8.24 \%$ \\
\hline Crossover & 1 & .03290 & $2.02 \%$ \\
\hline Crossover & Large & .4 & $100 \%$ \\
\hline Crossover & Medium & .25 & $100 \%$ \\
\hline Crossover & Small & .1 & $30.54 \%$ \\
\hline
\end{tabular}

Mean square error $=5.372283$ epochs. 


\section{Partitioned Sum of Squares}

Table A-9: F1-Partitioned sum of squares with 100 replicates

\begin{tabular}{|c|c|c|c|c|c|}
\hline Parameter & Df & Sum of Sq & Mean Sq & F Value & $\operatorname{Pr}(\mathrm{F})$ \\
\hline \hline Crossover & 6 & 12347 & 2057.83 & 8.4776 & $\mathbf{0 . 0 0 0 0 0 0 0}$ \\
\hline \multicolumn{6}{|c|}{ Crossover adjusted level of significance $=0.001673654$} \\
\hline Power of 1 & 1 & 10330 & 10329.82 & 42.5554 & $\mathbf{0 . 0 0 0 0 0 0 0}$ \\
\hline Power of 2 & 1 & 38 & 38.13 & 0.1571 & 0.6918712 \\
\hline Power of 3 & 1 & 976 & 975.98 & 4.0207 & 0.0449809 \\
\hline Power of 4 & 1 & 681 & 680.92 & 2.8052 & 0.0940032 \\
\hline Power of 5 & 1 & 14 & 13.70 & 0.0564 & 0.8122398 \\
\hline Power of 6 & 1 & 308 & 308.41 & 1.2705 & 0.2597008 \\
\hline \hline Mutation & 10 & 58701 & 5870.09 & 24.1828 & $\mathbf{0 . 0 0 0 0 0 0 0}$ \\
\hline Mutation adjusted level of significance $=0.001004529$ \\
\hline Power of 1 & 1 & 11389 & 11388.70 & 46.9176 & $\mathbf{0 . 0 0 0 0 0 0 0}$ \\
\hline Power of 2 & 1 & 44725 & 44724.56 & 184.2503 & $\mathbf{0 . 0 0 0 0 0 0 0}$ \\
\hline Power of 3 & 1 & 2 & 2.16 & 0.0089 & 0.9248439 \\
\hline Power of 4 & 1 & 1069 & 1068.68 & 4.4026 & 0.0359176 \\
\hline Power of 5 & 1 & 553 & 552.87 & 2.2776 & 0.1312950 \\
\hline Power of 6 & 1 & 452 & 451.55 & 1.8602 & 0.1726404 \\
\hline Power of 7 & 1 & 2 & 1.66 & 0.0068 & 0.9340925 \\
\hline Power of 8 & 1 & 487 & 486.78 & 2.0054 & 0.1567837 \\
\hline Power of 9 & 1 & 20 & 20.44 & 0.0842 & 0.7717104 \\
\hline Power of 10 & 1 & 4 & 3.52 & 0.0145 & 0.9041185 \\
\hline
\end{tabular}


Table A-10: F1-Partitioned sum of squares with 500 replicates

\begin{tabular}{|c|c|c|c|c|c|}
\hline Parameter & Df & Sum of Sq & Mean Sq & F Value & $\operatorname{Pr}(\mathrm{F})$ \\
\hline \hline Crossover & 6 & 82952 & 13825.4 & 56.2053 & $\mathbf{0 . 0 0 0 0 0 0 0}$ \\
\hline \multicolumn{6}{|c|}{ Crossover adjusted level of significance $=0.001673654$} \\
\hline Power of 1 & 1 & 82662 & 82661.9 & 336.0514 & $\mathbf{0 . 0 0 0 0 0 0 0}$ \\
\hline Power of 2 & 1 & 40 & 39.8 & 0.1619 & 0.6874415 \\
\hline Power of 3 & 1 & 31 & 31.2 & 0.1267 & 0.7219155 \\
\hline Power of 4 & 1 & 150 & 150.4 & 0.6116 & 0.4341996 \\
\hline Power of 5 & 1 & 17 & 16.5 & 0.0672 & 0.7954938 \\
\hline Power of 6 & 1 & 52 & 52.5 & 0.2132 & 0.6442386 \\
\hline \hline Mutation & 10 & 208227 & 20822.7 & 84.6522 & $\mathbf{0 . 0 0 0 0 0 0 0}$ \\
\hline \multicolumn{2}{|c|}{ Mutation adjusted level of significance =0.001004529 } \\
\hline Power of 1 & 1 & 32019 & 32018.7 & 130.1681 & $\mathbf{0 . 0 0 0 0 0 0 0}$ \\
\hline Power of 2 & 1 & 174262 & 174261.6 & 708.4383 & $\mathbf{0 . 0 0 0 0 0 0 0}$ \\
\hline Power of 3 & 1 & 959 & 959.3 & 3.9000 & 0.0482925 \\
\hline Power of 4 & 1 & 10 & 10.1 & 0.0409 & 0.8398032 \\
\hline Power of 5 & 1 & 108 & 107.8 & 0.4381 & 0.5080262 \\
\hline Power of 6 & 1 & 29 & 28.6 & 0.1162 & 0.7331794 \\
\hline Power of 7 & 1 & 350 & 349.8 & 1.4219 & 0.2330996 \\
\hline Power of 8 & 1 & 90 & 90.1 & 0.3663 & 0.5450536 \\
\hline Power of 9 & 1 & 344 & 344.1 & 1.3989 & 0.2369111 \\
\hline Power of 10 & 1 & 57 & 57.4 & 0.2335 & 0.6289593 \\
\hline
\end{tabular}


Table A-11: F1-Partitioned sum of squares of pooled analysis

\begin{tabular}{|c|c|c|c|c|c|}
\hline Parameter & Df & Sum of Sq & Mean Sq & F Value & $\operatorname{Pr}(\mathrm{F})$ \\
\hline \hline Crossover & 6 & 714.601 & 119.100 & 256.130 & $\mathbf{0 . 0 0 0 0 0 0 0}$ \\
\hline \multicolumn{6}{|c|}{ Crossover adjusted level of significance $=0.001673654$} \\
\hline Power of 1 & 1 & 708.852 & 708.852 & 1524.420 & $\mathbf{0 . 0 0 0 0 0 0 0}$ \\
\hline Power of 2 & 1 & 3.884 & 3.884 & 8.352 & 0.0041303 \\
\hline Power of 3 & 1 & 0.065 & 0.065 & 0.140 & 0.7082399 \\
\hline Power of 4 & 1 & 0.199 & 0.199 & 0.429 & 0.5131917 \\
\hline Power of 5 & 1 & 0.344 & 0.344 & 0.740 & 0.3904751 \\
\hline Power of 6 & 1 & 1.257 & 1.257 & 2.703 & 0.1011870 \\
\hline \hline Mutation & 10 & 2153.876 & 215.388 & 463.201 & $\mathbf{0 . 0 0 0 0 0 0 0}$ \\
\hline \multicolumn{7}{|c|}{ Mutation adjusted level of significance $=0.001004529$} \\
\hline Power of 1 & 1 & 473.173 & 473.173 & 1017.581 & $\mathbf{0 . 0 0 0 0 0 0 0}$ \\
\hline Power of 2 & 1 & 1665.259 & 1665.259 & 3581.217 & $\mathbf{0 . 0 0 0 0 0 0 0}$ \\
\hline Power of 3 & 1 & 6.476 & 6.476 & 13.926 & $\mathbf{0 . 0 0 0 2 2 6 9}$ \\
\hline Power of 4 & 1 & 3.828 & 3.828 & 8.232 & 0.0044039 \\
\hline Power of 5 & 1 & 2.830 & 2.830 & 6.087 & 0.0141682 \\
\hline Power of 6 & 1 & 0.397 & 0.397 & 0.854 & 0.3560224 \\
\hline Power of 7 & 1 & 0.984 & 0.984 & 2.116 & 0.1467925 \\
\hline Power of 8 & 1 & 0.760 & 0.760 & 1.634 & 0.2021186 \\
\hline Power of 9 & 1 & 0.154 & 0.154 & 0.330 & 0.5658050 \\
\hline Power of 10 & 1 & 0.015 & 0.015 & 0.031 & 0.8595995 \\
\hline
\end{tabular}


Table A-12: F3-Partitioned sum of squares of pooled analysis

\begin{tabular}{|c|c|c|c|c|c|}
\hline Parameter & Df & Sum of Sq & Mean Sq & F Value & $\operatorname{Pr}(\mathrm{F})$ \\
\hline \hline Crossover & 4 & 196.365 & 49.091 & 34.871 & $\mathbf{0 . 0 0 0 0 0 0 0}$ \\
\hline \multicolumn{6}{|c|}{ Crossover adjusted level of significance $=0.00250943$} \\
\hline Power of 1 & 1 & 191.806 & 191.806 & 136.247 & $\mathbf{0 . 0 0 0 0 0 0 0}$ \\
\hline Power of 2 & 1 & 0.773 & 0.773 & 0.549 & 0.4596335 \\
\hline Power of 3 & 1 & 1.118 & 1.118 & 0.794 & 0.3740606 \\
\hline Power of 4 & 1 & 2.668 & 2.668 & 1.895 & 0.1703326 \\
\hline \hline Mutation & 8 & 3520.036 & 440.004 & 312.551 & $\mathbf{0 . 0 0 0 0 0 0 0}$ \\
\hline Mutation adjusted level of significance =0.001255503 \\
\hline Power of 1 & 1 & 127.126 & 127.126 & 90.302 & $\mathbf{0 . 0 0 0 0 0 0 0}$ \\
\hline Power of 2 & 1 & 3377.901 & 3377.901 & 2399.447 & $\mathbf{0 . 0 0 0 0 0 0 0}$ \\
\hline Power of 3 & 1 & 6.795 & 6.795 & 4.827 & 0.0293291 \\
\hline Power of 4 & 1 & 4.257 & 4.257 & 3.024 & 0.0837819 \\
\hline Power of 5 & 1 & 2.047 & 2.047 & 1.454 & 0.2294650 \\
\hline
\end{tabular}


Table A-13: F2-Partitioned sum of squares of pooled analysis

\begin{tabular}{|c|c|c|c|c|c|}
\hline Parameter & Df & Sum of Sq & Mean Sq & F Value & $\operatorname{Pr}(\mathrm{F})$ \\
\hline Crossover & 14 & 29291.3 & 2092.24 & 46.109 & 0.0000000 \\
\hline \multicolumn{6}{|c|}{ Crossover adjusted level of significance $=0.0007176235$} \\
\hline Power of 1 & 1 & 28663.0 & 28662.98 & 631.676 & 0.0000000 \\
\hline Power of 2 & 1 & 149.4 & 149.43 & 3.293 & 0.0699523 \\
\hline Power of 3 & 1 & 60.2 & 60.24 & 1.328 & 0.2495765 \\
\hline Power of 4 & 1 & 62.7 & 62.66 & 1.381 & 0.2403146 \\
\hline Power of 5 & 1 & 0.1 & 0.07 & 0.002 & 0.9677584 \\
\hline Power of 6 & 1 & 96.2 & 96.19 & 2.120 & 0.1458023 \\
\hline Power of 7 & 1 & 5.3 & 5.33 & 0.118 & 0.7318478 \\
\hline Power of 8 & 1 & 64.0 & 64.01 & 1.411 & 0.2353115 \\
\hline Power of 9 & 1 & 30.2 & 30.15 & 0.665 & 0.4152246 \\
\hline Power of 10 & 1 & 73.4 & 73.37 & 1.617 & 0.2039037 \\
\hline Power of 11 & 1 & 27.2 & 27.20 & 0.599 & 0.4390594 \\
\hline Power of 12 & 1 & 12.3 & 12.28 & 0.271 & 0.6030844 \\
\hline Power of 13 & 1 & 43.8 & 43.83 & 0.966 & 0.3259990 \\
\hline Power of 14 & 1 & 3.5 & 3.54 & 0.078 & 0.7799435 \\
\hline Mutation & 12 & 103575.8 & 8631.32 & 190.217 & 0.0000000 \\
\hline \multicolumn{6}{|c|}{ Mutation adjusted level of significance $=0.0008371774$} \\
\hline Power of 1 & 1 & 3878.8 & 3878.80 & 85.481 & 0.0000000 \\
\hline Power of 2 & 1 & 96213.2 & 96213.19 & 2120.350 & 0.0000000 \\
\hline Power of 3 & 1 & 2662.8 & 2662.77 & 58.682 & 0.0000000 \\
\hline Power of 4 & 1 & 20.8 & 20.84 & 0.459 & 0.4982083 \\
\hline Power of 5 & 1 & 13.5 & 13.46 & 0.297 & 0.5862050 \\
\hline Power of 6 & 1 & 172.7 & 172.68 & 3.805 & 0.0514453 \\
\hline Power of 7 & 1 & 5.3 & 5.31 & 0.117 & 0.7323648 \\
\hline Power of 8 & 1 & 72.0 & 72.03 & 1.587 & 0.2080834 \\
\hline Power of 9 & 1 & 116.6 & 116.57 & 2.569 & 0.1093895 \\
\hline Power of 10 & 1 & 57.4 & 57.37 & 1.264 & 0.2611975 \\
\hline Power of 11 & 1 & 343.5 & 343.54 & 7.571 & 0.0060701 \\
\hline Power of 12 & 1 & 19.3 & 19.26 & 0.424 & 0.5149314 \\
\hline
\end{tabular}


Table A-14: F2-Partitioned sum of squares of pooled analysis continued

\begin{tabular}{|l|c|c|c|c|c|}
\hline Parameter & Df & Sum of Sq & Mean Sq & F Value & $\operatorname{Pr}(\mathrm{F})$ \\
\hline \hline Interaction & 168 & 10717.5 & 63.79 & 1.406 & $\mathbf{0 . 0 0 1 5 5 0 1}$ \\
\hline \multicolumn{7}{|c|}{ Interaction adjusted level of significance = 0.00005982164. } \\
\hline $\begin{array}{l}\text { Power of 1: } \\
\text { Power of 1 }\end{array}$ & 1 & 2924.0 & 2923.96 & 64.438 & $\mathbf{0 . 0 0 0 0 0 0 0}$ \\
\hline
\end{tabular}


Table A-15: F6-Partitioned sum of squares of pooled analysis

\begin{tabular}{|c|c|c|c|c|c|}
\hline Parameter & Df & Sum of $\mathrm{Sq}$ & Mean Sq & F Value & $\operatorname{Pr}(\mathrm{F})$ \\
\hline Crossover & 14 & 54420.8 & 3887.2 & 93.454 & 0.0000000 \\
\hline \multicolumn{6}{|c|}{ Crossover adjusted level of significance $=0.0007176235$} \\
\hline Power of 1 & 1 & 51558.8 & 51558.8 & 1239.544 & 0.0000000 \\
\hline Power of 2 & 1 & 2723.0 & 2723.0 & 65.465 & 0.0000000 \\
\hline Power of 3 & 1 & 0.1 & 0.1 & 0.002 & 0.9672032 \\
\hline Power of 4 & 1 & 0.2 & 0.2 & 0.005 & 0.9438726 \\
\hline Power of 5 & 1 & 14.2 & 14.2 & 0.340 & 0.5597281 \\
\hline Power of 6 & 1 & 10.2 & 10.2 & 0.246 & 0.6203542 \\
\hline Power of 7 & 1 & 5.0 & 5.0 & 0.121 & 0.7282759 \\
\hline Power of 8 & 1 & 17.3 & 17.3 & 0.417 & 0.5187929 \\
\hline Power of 9 & 1 & 59.5 & 59.5 & 1.430 & 0.2321141 \\
\hline Power of 10 & 1 & 1.7 & 1.7 & 0.040 & 0.8419240 \\
\hline Power of 11 & 1 & 0.0 & 0.0 & 0.000 & 0.9855772 \\
\hline Power of 12 & 1 & 0.1 & 0.1 & 0.002 & 0.9613900 \\
\hline Power of 13 & 1 & 30.7 & 30.7 & 0.739 & 0.3901418 \\
\hline Power of 14 & 1 & 0.0 & 0.0 & 0.000 & 0.9893777 \\
\hline
\end{tabular}


Table A-16: F6-Partitioned sum of squares of pooled analysis continued

\begin{tabular}{|c|c|c|c|c|c|}
\hline Parameter & Df & Sum of Sq & Mean Sq & F Value & $\operatorname{Pr}(\mathrm{F})$ \\
\hline Mutation & 14 & 162014.1 & 11572.4 & 278.217 & 0.0000000 \\
\hline \multicolumn{6}{|c|}{ Mutation adjusted level of significance $=0.0007176235$} \\
\hline Power of 1 & 1 & 49729.9 & 49729.9 & 1195.574 & 0.0000000 \\
\hline Power of 2 & 1 & 111146.3 & 111146.3 & 2672.109 & 0.0000000 \\
\hline Power of 3 & 1 & 485.9 & 485.9 & 11.681 & 0.0006599 \\
\hline Power of 4 & 1 & 209.9 & 209.9 & 5.047 & 0.0249066 \\
\hline Power of 5 & 1 & 42.7 & 42.7 & 1.027 & 0.3112273 \\
\hline Power of 6 & 1 & 26.7 & 26.7 & 0.641 & 0.4233990 \\
\hline Power of 7 & 1 & 245.7 & 245.7 & 5.908 & 0.0152684 \\
\hline Power of 8 & 1 & 52.5 & 52.5 & 1.263 & 0.2613394 \\
\hline Power of 9 & 1 & 35.8 & 35.8 & 0.861 & 0.3538391 \\
\hline Power of 10 & 1 & 31.1 & 31.1 & 0.749 & 0.3871409 \\
\hline Power of 11 & 1 & 4.8 & 4.8 & 0.116 & 0.7339592 \\
\hline Power of 12 & 1 & 0.1 & 0.1 & 0.003 & 0.9595070 \\
\hline Power of 13 & 1 & 1.8 & 1.8 & 0.043 & 0.8351457 \\
\hline Power of 14 & 1 & 0.8 & 0.8 & 0.019 & 0.8895168 \\
\hline
\end{tabular}


Table A-17: F6-Partitioned sum of squares of pooled analysis continued

\begin{tabular}{|c|c|c|c|c|c|}
\hline Parameter & Df & Sum of Sq & Mean Sq & F Value & $\operatorname{Pr}(\mathrm{F})$ \\
\hline \hline Interaction & 196 & 50461.5 & 257.5 & 6.190 & $\mathbf{0 . 0 0 0 0 0 0 0}$ \\
\hline \multicolumn{7}{|c|}{ Interaction adjusted level of significance = 0.00005127591. } \\
Only significant results shown. \\
\hline $\begin{array}{c}\text { Power of 1: } \\
\text { Power of 1 }\end{array}$ & 1 & 34688.8 & 34688.8 & 833.966 & $\mathbf{0 . 0 0 0 0 0 0 0}$ \\
\hline $\begin{array}{c}\text { Power of 2: } \\
\text { Power of 1 }\end{array}$ & 1 & 1464.2 & 1464.2 & 35.200 & $\mathbf{0 . 0 0 0 0 0 0 0}$ \\
\hline $\begin{array}{c}\text { Power of 1: } \\
\text { Power of 2 }\end{array}$ & 1 & 5426.3 & 5426.3 & 130.457 & $\mathbf{0 . 0 0 0 0 0 0 0}$ \\
\hline $\begin{array}{c}\text { Power of 1: } \\
\text { Power of 3 }\end{array}$ & 1 & 925.8 & 925.8 & 22.257 & $\mathbf{0 . 0 0 0 0 0 2 8}$ \\
\hline
\end{tabular}


Table A-18: F6-Partitioned sum of squares of pooled analysis for crossover

\begin{tabular}{|c|c|c|c|c|c|}
\hline Parameter & Df & Sum of Sq & Mean Sq & F Value & $\operatorname{Pr}(\mathrm{F})$ \\
\hline Crossover & 15 & 818.36 & 54.56 & 1.890 & 0.0207598 \\
\hline \multicolumn{6}{|c|}{ Crossover adjusted level of significance $=0.000669798$} \\
\hline Power of 1 & 1 & 381.88 & 381.88 & 13.232 & 0.0002900 \\
\hline Power of 2 & 1 & 7.33 & 7.33 & 0.254 & 0.6143782 \\
\hline Power of 3 & 1 & 0.68 & 0.68 & 0.024 & 0.8778748 \\
\hline Power of 4 & 1 & 54.75 & 54.75 & 1.897 & 0.1687276 \\
\hline Power of 5 & 1 & 37.90 & 37.90 & 1.313 & 0.2520953 \\
\hline Power of 6 & 1 & 35.89 & 35.89 & 1.243 & 0.2650954 \\
\hline Power of 7 & 1 & 1.05 & 1.05 & 0.037 & 0.8484232 \\
\hline Power of 8 & 1 & 23.91 & 23.91 & 0.828 & 0.3629396 \\
\hline Power of 9 & 1 & 3.03 & 3.03 & 0.105 & 0.7461390 \\
\hline Power of 10 & 1 & 0.10 & 0.10 & 0.003 & 0.9528493 \\
\hline Power of 11 & 1 & 18.28 & 18.28 & 0.634 & 0.4262661 \\
\hline Power of 12 & 1 & 50.86 & 50.86 & 1.762 & 0.1846610 \\
\hline Power of 13 & 1 & 193.18 & 193.18 & 6.693 & 0.0098245 \\
\hline Power of 14 & 1 & 4.52 & 4.52 & 0.156 & 0.6925059 \\
\hline Power of 15 & 1 & 4.99 & 4.99 & 0.173 & 0.6776497 \\
\hline
\end{tabular}




\section{Fitted response curves}

Table A-19: Equations of fitted response curves

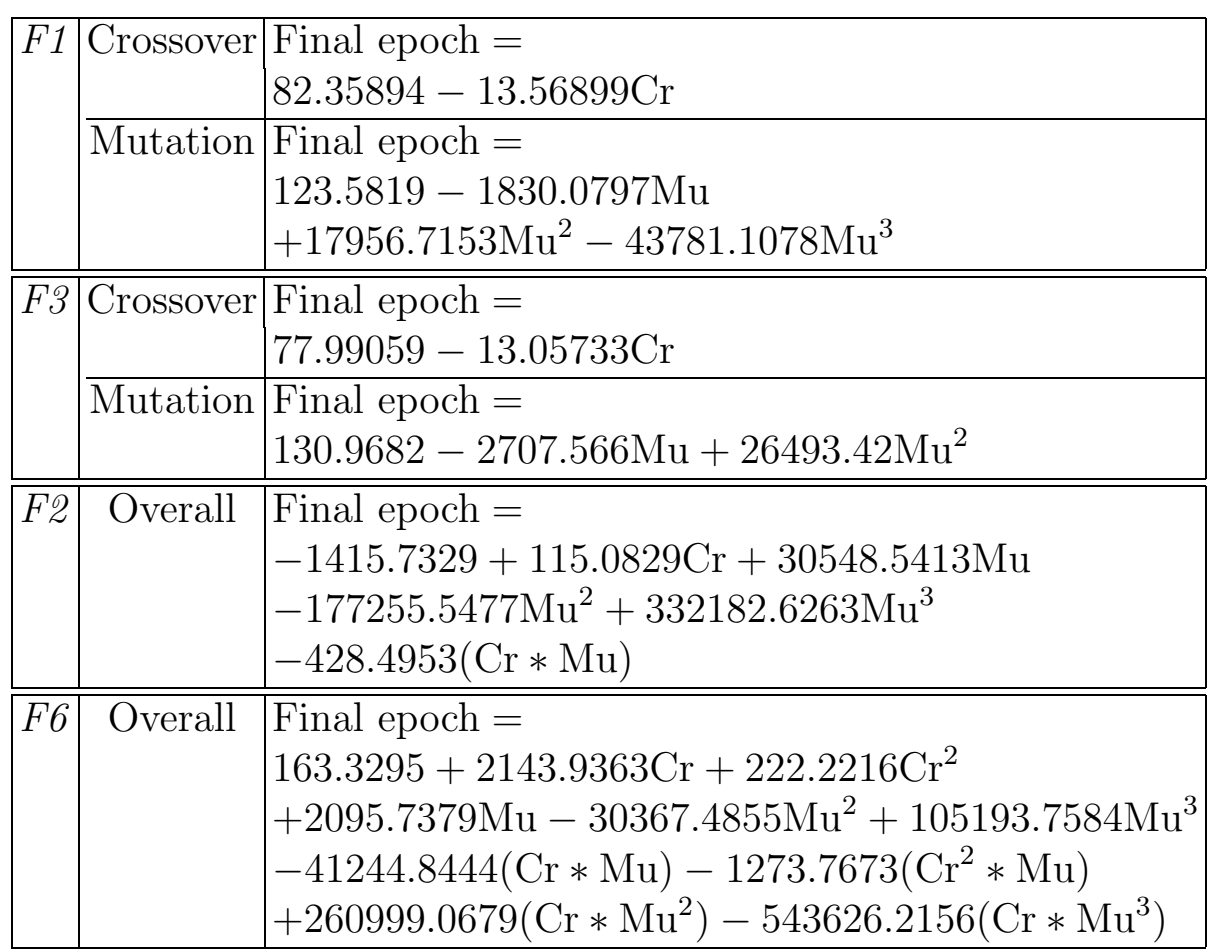

Crossover parameter level $(\mathrm{Cr})$, Mutation parameter level $(\mathrm{Mu})$. 


\title{
Appendix B
}

\section{FN1 to FN6}

\author{
B Results
}

\section{ANOVA Tables}

Table B-1: ANOVA results of $F N 1$

\begin{tabular}{|c|c|c|c|c|c|}
\hline \multicolumn{7}{|c|}{ Test function $\boldsymbol{F N 1}$} \\
\hline \multicolumn{7}{|c|}{ Crossover: 0.7 to 1 with an interval of 0.05} \\
\hline Mutation: 0.07 to 0.11 with interval of 0.005 \\
\hline \hline Parameter & Df & Sum of Sq & Mean Sq & F Value & p-value \\
\hline Mutation & 8 & 195.0530 & 24.38163 & 71.1810 & 0.0000000 \\
\hline Interaction & 48 & 12.5655 & 0.26178 & 0.7643 & 0.8678564 \\
\hline Block & 4 & 5.7498 & 1.43745 & 4.1966 & 0.0026330 \\
\hline Residuals & 248 & 84.9475 & 0.34253 & - & - \\
\hline \multicolumn{7}{|c|}{ Residual standard error: 0.5852608, Power $=87.03 \%}$. \\
\hline
\end{tabular}


Table B-2: ANOVA results of FN2 and FN3

\begin{tabular}{|c|c|c|c|c|c|}
\hline \multicolumn{7}{|c|}{ Test function FN2 } \\
\hline \multicolumn{7}{|c|}{ Crossover: 0.8 to 1 with an interval of 0.05 } \\
\hline Mutation: 0.07 to 0.11 with an interval of 0.005 \\
\hline Parameter & Df & Sum of Sq & Mean Sq & F Value & p-value \\
\hline Crossover & 4 & 79.23721 & 19.80930 & 66.65568 & 0.0000000 \\
\hline Mutation & 8 & 91.09235 & 11.38654 & 38.31421 & 0.0000000 \\
\hline Interaction & 32 & 9.95044 & 0.31095 & 1.04631 & 0.4066007 \\
\hline Block & 5 & 1.74695 & 0.34939 & 1.17565 & 0.3220536 \\
\hline Residuals & 220 & 65.38147 & 0.29719 & - & - \\
\hline Residual standard error: 0.54515, Power $=88.24 \%$. \\
\hline \hline \multicolumn{7}{|c|}{ Test function FN3 } \\
\hline Crossover: 0.4 to 1 with an interval of 0.05 \\
\hline Mutation: 0.16 to 0.19 with an interval of 0.005 \\
\hline Parameter & Df & Sum of Sq & Mean Sq & F Value & p-value \\
\hline \hline Crossover & 12 & 14002.2 & 1166.85 & 3.9242 & 0.00001088 \\
\hline Mutation & 6 & 313701.8 & 52283.64 & 175.8325 & 0.00000000 \\
\hline Interaction & 72 & 31744.0 & 440.89 & 1.4827 & 0.01105187 \\
\hline Block & 4 & 5179.7 & 1294.94 & 4.3549 & 0.00188308 \\
\hline Residuals & 360 & 107045.7 & 297.35 & - & - \\
\hline Residual standard error: 17.24381, Power $=95.96 \%$. \\
\hline
\end{tabular}


Table B-3: ANOVA results of FN4 and FN5

\begin{tabular}{|c|c|c|c|c|c|}
\hline \multicolumn{7}{|c|}{ Test function FN4 } \\
\hline \multicolumn{7}{|c|}{ Crossover: 0.7 to 1 with an interval of 0.05 } \\
\hline Parameter & Df & Sum of Sq & Mean Sq & F Value & p-value \\
\hline Crossover & 6 & 169.0313 & 28.17188 & 93.23987 & 0.0000000 \\
\hline Mutation & 8 & 131.1151 & 16.38938 & 54.24359 & 0.0000000 \\
\hline Interaction & 48 & 10.1115 & 0.21066 & 0.69720 & 0.9329824 \\
\hline Block & 4 & 4.9472 & 1.23681 & 4.09345 & 0.0031292 \\
\hline Residuals & 248 & 74.9318 & 0.30214 & - & - \\
\hline Residual standard error: 0.5496764, Power $=87.03 \%$. \\
\hline \hline \multicolumn{7}{|c|}{ Test function FN5 } \\
\hline Crossover: 0.1 to 1 with an interval of 0.05 \\
\hline Mutation: 0.12 to 0.16 with an interval of 0.005 \\
\hline Parameter & Df & Sum of Sq & Mean Sq & F Value & p-value \\
\hline \hline Crossover & 18 & 5566.06 & 309.225 & 46.8718 & 0.00000000 \\
\hline Mutation & 8 & 18131.18 & 2266.398 & 343.5364 & 0.00000000 \\
\hline Interaction & 144 & 1558.08 & 10.820 & 1.6401 & $\mathbf{0 . 0 0 0 0 2 6 6 3}$ \\
\hline Block & 4 & 54.90 & 13.724 & 2.0802 & 0.08175970 \\
\hline Residuals & 680 & 4486.13 & 6.597 & - & - \\
\hline Residual standard error: 2.568512, Power $=99.90 \%$. \\
\hline
\end{tabular}


Table B-4: ANOVA results of FN6

\begin{tabular}{|c|c|c|c|c|c|}
\hline \multicolumn{7}{|c|}{ Test function $\boldsymbol{F N 6}$} \\
\hline \multicolumn{7}{|c|}{ Crossover: 0.1 to 1 with an interval of 0.05} \\
\hline Parameter & Df & Sum of Sq & Mean Sq & F Value & p-value \\
\hline \hline Crossover & 18 & 207154 & 11509 & 4.0106 & 0.000000057 \\
\hline Mutation & 8 & 16671466 & 2083933 & 726.2203 & 0.000000000 \\
\hline Interaction & 144 & 736294 & 5113 & 1.7819 & $\mathbf{0 . 0 0 0 0 0 1 0 0 8}$ \\
\hline Block & 4 & 41181 & 10295 & 3.5878 & 0.006617639 \\
\hline Residuals & 680 & 1951301 & 2870 & - & - \\
\hline \multicolumn{7}{|c|}{ Residual standard error: 53.56828, Power $=99.90 \%}$. \\
\hline
\end{tabular}




\section{Fitted response curves}

Table B-5: Equations of fitted response curves for FN1 to FN6

\begin{tabular}{|c|c|c|}
\hline$F N 1$ & Mutation & $\begin{array}{l}\text { Final epoch }= \\
56.97715-8.15829 \mathrm{Cr} \\
\text { Final epoch }= \\
81.23346-745.06687 \mathrm{Mu} \\
+4338.52814 \mathrm{Mu}^{2}\end{array}$ \\
\hline FN2 & \begin{tabular}{|l|} 
Crossover \\
Mutation
\end{tabular} & $\begin{array}{l}\text { Final epoch }= \\
56.9028000-7.6368889 \mathrm{Cr} \\
\text { Final epoch }= \\
7.877 \times 10^{1}-6.652 \times 10^{2} \mathrm{Mu} \\
+3.765 \times 10^{3} \mathrm{Mu}^{2}\end{array}$ \\
\hline FN3 & Mutation & $\begin{array}{l}\text { Final epoch }= \\
454.9500-26.0478 \mathrm{Cr} \\
\text { Final epoch }= \\
9.540 \times 10^{3}-1.047 \times 10^{5} \mathrm{Mu} \\
+2.999 \times 10^{5} \mathrm{Mu}^{2} \\
\end{array}$ \\
\hline FN4 & \begin{tabular}{|l} 
Crossover \\
Mutation
\end{tabular} & $\begin{array}{l}\text { Final epoch }= \\
51.395690-7.307079 \mathrm{Cr} \\
\text { Final epoch }= \\
6.958 \times 10^{1}-5.954 \times 10^{2} \mathrm{Mu} \\
+3.539 \times 10^{3} \mathrm{Mu}^{2}\end{array}$ \\
\hline$F N 5$ & Overall & $\begin{array}{l}\text { Final epoch }= \\
-218.5247+16.10332 \mathrm{Cr}+8.586955 \mathrm{Cr}^{2} \\
+11631.9485 \mathrm{Mu}-113700.7892 \mathrm{Mu}^{2} \\
+344700.9038 \mathrm{Mu}^{3}-246.3479(\mathrm{Cr} * \mathrm{Mu})\end{array}$ \\
\hline$F N 6$ & Overall & $\begin{array}{l}\text { Final epoch }= \\
-3731.3012+892.2784 \mathrm{Cr}+237189.8786 \mathrm{Mu} \\
-2052110.9896 \mathrm{Mu}^{2}+4964206.9821 \mathrm{Mu}^{3} \\
-4941.4196(\mathrm{Cr} * \mathrm{Mu})\end{array}$ \\
\hline
\end{tabular}

Crossover parameter level (Cr), Mutation parameter level $(\mathrm{Mu})$. 


\section{Polynomial Regression Tables}

Table B-6: Polynomial regression of FN1 to FN4

\begin{tabular}{|c|c|c|c|}
\hline \multicolumn{4}{|c|}{ Test function $F N 1$} \\
\hline Parameter 1 & Response curve shape & Estimated best rate & $99 \% \mathrm{CI}$ \\
\hline Crossover & Linear & $100 \%$ & - \\
\hline Mutation & Quadratic & $8.59 \%$ & $8.43 \%-8.75 \%$ \\
\hline Interaction & Nil & - & - \\
\hline \multicolumn{4}{|c|}{ Test function $F N 2$} \\
\hline Parameter 1 & Response curve shape & Estimated best rate & $99 \% \mathrm{CI}$ \\
\hline Crossover & Linear & $100 \%$ & - \\
\hline Mutation & Quadratic & $8.83 \%$ & $8.70 \%-8.96 \%$ \\
\hline Interaction & Nil & - & - \\
\hline \multicolumn{4}{|c|}{ Test function $F N 3$} \\
\hline Parameter 1 & Response curve shape & Estimated best rate & $99 \% \mathrm{CI}$ \\
\hline Crossover & Linear & $100 \%$ & - \\
\hline Mutation & Quadratic & $17.45 \%$ & $17.41 \%-17.49 \%$ \\
\hline Interaction & Nil & - & - \\
\hline \multicolumn{4}{|c|}{ Test function $F N_{4}$} \\
\hline Parameter 1 & Response curve shape & Estimated best rate & $99 \% \mathrm{CI}$ \\
\hline Crossover & Linear & $100 \%$ & - \\
\hline Mutation & Quadratic & $8.41 \%$ & $8.23 \%-8.59 \%$ \\
\hline Interaction & Nil & - & - \\
\hline
\end{tabular}


Table B-7: Polynomial regression of FN5 and FN6

\begin{tabular}{|c|c|c|c|}
\hline \multicolumn{4}{|c|}{ Test function $\boldsymbol{F N 5}$} \\
\hline Parameter & Response curve shape & Estimated best rate & $99 \%$ CI \\
\hline \hline Crossover & Quadratic & $100 \%$ & - \\
\hline Mutation & Cubic & $14.11 \%$ & $14.01 \%-14.21 \%$ \\
\hline Interaction & Linear:Linear & - & - \\
\hline \hline \multicolumn{4}{|c|}{ Test function $\mathbf{F N 6}$} \\
\hline Parameter & Response curve shape & Estimated best rate & $99 \%$ CI \\
\hline \hline Crossover & Linear & $100 \%$ & - \\
\hline Mutation & Cubic & $19.47 \%$ & $19.42 \%-19.53 \%$ \\
\hline Interaction & Linear:Linear & - & - \\
\hline
\end{tabular}




\title{
Appendix C
}

\section{FN1R45 to FN6R45 and}

\section{Landscape $\_20 \_101$}

\author{
C Results
}

\section{ANOVA Tables}

Table C-1: ANOVA results of FN1R 45

\begin{tabular}{|c|c|c|c|c|c|}
\hline \multicolumn{7}{|c|}{ Test function FN1R45 } \\
\hline Crossover: 0.6 to 1 with an interval of 0.05 \\
\hline Mutation: 0.07 to 0.12 with an interval of 0.005 \\
\hline Parameter & Df & Sum of Sq & Mean Sq & F Value & p-value \\
\hline \hline Crossover & 8 & 596.70 & 74.59 & 101.9350 & $<2 \times 10^{-16}$ \\
\hline Mutation & 10 & 1551.30 & 155.13 & 212.0105 & $<2 \times 10^{-16}$ \\
\hline Interaction & 80 & 54.96 & 0.69 & 0.9389 & 0.6263 \\
\hline Block & 4 & 2.12 & 0.53 & 0.7242 & 0.5758 \\
\hline Residuals & 392 & 286.83 & 0.73 & - & - \\
\hline Residual standard error=0.8554008, Power $=97.02 \%$, Threshold=7. \\
\hline
\end{tabular}


Table C-2: ANOVA results of FN1R45 and FN2R45

\begin{tabular}{|c|c|c|c|c|c|}
\hline \multicolumn{6}{|c|}{ Test function FN2R45 } \\
\hline \multicolumn{5}{|c|}{ Crossover: 0 to 0.4 with an interval of 0.05 } \\
\hline Mutation: 0.23 to 0.28 with an interval of 0.005 \\
\hline Parameter & Df & Sum of Sq & Mean Sq & F Value & p-value \\
\hline \hline Crossover & 8 & 691359 & 86420 & 30.6658 & $<2.2 \times 10^{-16}$ \\
\hline Mutation & 10 & 7590923 & 759092 & 269.3608 & $<2.2 \times 10^{-16}$ \\
\hline Interaction & 80 & 422004 & 5275 & 1.8718 & $4.963 \times 10^{-05}$ \\
\hline Block & 4 & 12955 & 3239 & 1.1493 & 0.3329 \\
\hline Residuals & 392 & 1104705 & 2818 & - & - \\
\hline Residual standard error=53.08601, Power=97.02\%, Threshold=6. \\
\hline \hline \multicolumn{7}{|c|}{ Test function FN3R45 } \\
\hline Crossover: 0 to 1 with an interval of 0.05 \\
\hline Mutation: 0.11 to 0.14 with an interval of 0.005 \\
\hline Parameter & Df & Sum of Sq & Mean Sq & F Value & p-value \\
\hline \hline Crossover & 20 & 942.53 & 47.13 & 11.0612 & $<2.2 \times 10^{-16}$ \\
\hline Mutation & 6 & 2235.96 & 372.66 & 87.4686 & $<2.2 \times 10^{-16}$ \\
\hline Interaction & 120 & 844.28 & 7.04 & 1.6514 & $8.505 \times 10^{-05}$ \\
\hline Block & 4 & 69.94 & 17.48 & 4.1039 & 0.002742 \\
\hline Residuals & 584 & 2488.14 & 4.26 & - & - \\
\hline Residual standard error=2.064100, Power=99.71\%, Threshold=7. \\
\hline
\end{tabular}


Table C-3: ANOVA results of FN4R45 and FN5R45

\begin{tabular}{|c|c|c|c|c|c|}
\hline \multicolumn{7}{|c|}{ Test function FN4R45 } \\
\hline \multicolumn{6}{|c|}{ Crossover: 0 to 0.8 with an interval of 0.05 } \\
\hline Mutation: 0.33 to 0.37 with an interval of 0.005 \\
\hline Parameter & Df & Sum of Sq & Mean Sq & F Value & p-value \\
\hline \hline Crossover & 16 & 1159371 & 72461 & 61.5758 & $<2.2 \times 10^{-16}$ \\
\hline Mutation & 8 & 1968603 & 246075 & 209.1107 & $<2.2 \times 10^{-16}$ \\
\hline Interaction & 128 & 402189 & 3142 & 2.6701 & $1.458 \times 10^{-15}$ \\
\hline Block & 4 & 6601 & 1650 & 1.4022 & 0.2317 \\
\hline Residuals & 608 & 715477 & 1177 & - & - \\
\hline Residual standard error=34.30410, Power=99.76\%, Threshold=5. \\
\hline \hline \multicolumn{7}{|c|}{ Test function FN5R45 } \\
\hline Crossover: 0 to 0.5 with an interval of 0.05 \\
\hline Mutation: 0.3 to 0.35 with an interval of 0.005 \\
\hline Parameter & Df & Sum of Sq & Mean Sq & F Value & p-value \\
\hline \hline Crossover & 10 & 756983 & 75698 & 84.9871 & $<2.2 \times 10^{-16}$ \\
\hline Mutation & 10 & 3162538 & 316254 & 355.0607 & $<2.2 \times 10^{-16}$ \\
\hline Interaction & 100 & 186328 & 1863 & 2.0919 & $1.301 \times 10^{-07}$ \\
\hline Block & 4 & 710 & 178 & 0.1994 & 0.9386 \\
\hline Residuals & 480 & 427538 & 891 & - & - \\
\hline Residual standard error=29.84466, Power=98.86\%, Threshold=5. \\
\hline
\end{tabular}


Table C-4: ANOVA results of FN6R45 and Landscape_20_101

\begin{tabular}{|c|c|c|c|c|c|}
\hline \multicolumn{7}{|c|}{ Test function FN6R45 } \\
\hline \multicolumn{7}{|c|}{ Crossover: 0 to 1 with an interval of 0.05 } \\
\hline Mutation: 0.09 to 0.15 with an interval of 0.005 \\
\hline \hline Crossover & 20 & 12649 & 632 & 52.6177 & $<2 \times 10^{-16}$ \\
\hline Mutation & 12 & 269824 & 22485 & 1870.6825 & $<2 \times 10^{-16}$ \\
\hline Interaction & 240 & 25698 & 107 & 8.9080 & $<2 \times 10^{-16}$ \\
\hline Block & 4 & 111 & 28 & 2.3052 & 0.05652 \\
\hline Residuals & 1088 & 13078 & 12 & - & - \\
\hline Residual standard error=3.466965, Power=100\%, Threshold=7. \\
\hline \hline \multicolumn{7}{|c|}{ Test function Landscape_20_101 } \\
\hline Crossover: 0 to 1 with an interval of 0.1 \\
\hline Mutation: 0.15 to 0.21 with an interval of 0.01 \\
\hline Parameter & Df & Sum of Sq & Mean Sq & F Value & p-value \\
\hline \hline Crossover & 10 & 897763 & 89776 & 21.2996 & $<2 \times 10^{-16}$ \\
\hline Mutation & 6 & 11679219 & 1946536 & 461.8201 & $<2 \times 10^{-16}$ \\
\hline Interaction & 60 & 1059207 & 17653 & 4.1883 & $<2 \times 10^{-16}$ \\
\hline Block & 4 & 33611 & 8403 & 1.9936 & 0.09541 \\
\hline Residuals & 304 & 1281337 & 4215 & - & - \\
\hline Residual standard error=64.92244, Power=92.65\%, Threshold=5. \\
\hline
\end{tabular}




\section{Fitted response curves}

Table C-5: Equations of fitted response curves for FN1R45 to FN6R45

\begin{tabular}{|c|c|c|}
\hline \multirow[t]{2}{*}{ FN1R45 } & Crossover & $\begin{array}{l}\text { Final epoch }= \\
70.410317-8.471164 \mathrm{Cr}\end{array}$ \\
\hline & Mutation & $\begin{array}{l}\text { Final epoch }= \\
1.048 \times 10^{2}-9.756 \times 10^{2} \mathrm{Mu} \\
+5.556 \times 10^{3} \mathrm{Mu}^{2}\end{array}$ \\
\hline$F N 2 R 45$ & Overall & $\begin{array}{l}\text { Final epoch }= \\
3.666 \times 10^{4}+3.283 \times 10^{3} \mathrm{Cr}-2.811 \times 10^{5} \mathrm{Mu} \\
+5.569 \times 10^{5} \mathrm{Mu}^{2}-1.174 \times 10^{4}(\mathrm{Cr} * \mathrm{Mu})\end{array}$ \\
\hline$F N 3 R 45$ & Overall & $\begin{array}{l}\text { Final epoch }= \\
1.228 \times 10^{4}+2.619 \times 10^{1} \mathrm{Cr}+9.058 \mathrm{Cr}^{2} \\
-3.854 \times 10^{5} \mathrm{Mu}+4.577 \times 10^{6} \mathrm{Mu}^{2} \\
-2.419 \times 10^{7} \mathrm{Mu}^{3}+4.801 \times 10^{7} \mathrm{Mu}^{4} \\
-2.605 \times 10^{2}(\mathrm{Cr} * \mathrm{Mu})\end{array}$ \\
\hline$F_{4} R_{4} 5$ & Overall & $\begin{array}{l}\text { Final epoch }= \\
-1.260 \times 10^{5}+2.234 \times 10^{3} \mathrm{Cr}+1.203 \times 10^{6} \mathrm{Mu} \\
-3.768 \times 10^{6} \mathrm{Mu}^{2}+3.906 \times 10^{6} \mathrm{Mu}^{3} \\
-5.934 \times 10^{3}(\mathrm{Cr} * \mathrm{Mu})\end{array}$ \\
\hline$F N 5 R 45$ & Overall & $\begin{array}{l}\text { Final epoch }= \\
-6.428 \times 10^{4}+1.858 \times 10^{3} \mathrm{Cr}+6.774 \times 10^{5} \mathrm{Mu} \\
-2.316 \times 10^{6} \mathrm{Mu}^{2}+2.602 \times 10^{6} \mathrm{Mu}^{3} \\
-5.032 \times 10^{3}(\mathrm{Cr} * \mathrm{Mu})\end{array}$ \\
\hline$F N 6 R 45$ & Overall & $\begin{array}{l}\text { Final epoch }= \\
1.177 \times 10^{3}+7.129 \times 10^{2} \mathrm{Cr}+5.974 \times 10^{1} \mathrm{Cr}^{2} \\
-3.074 \times 10^{4} \mathrm{Mu}+3.463 \times 10^{5} \mathrm{Mu}^{2} \\
-1.835 \times 10^{6} \mathrm{Mu}^{3}+3.845 \times 10^{6} \mathrm{Mu}^{4} \\
-1.633 \times 10^{4}(\mathrm{Cr} * \mathrm{Mu})-4.103 \times 10^{2}\left(\mathrm{Cr}^{2} * \mathrm{Mu}\right) \\
+1.232 \times 10^{5}\left(\mathrm{Cr} * \mathrm{Mu}^{2}\right)-3.084 \times 10^{5}\left(\mathrm{Cr} * \mathrm{Mu}^{3}\right)\end{array}$ \\
\hline
\end{tabular}

Crossover parameter level (Cr), Mutation parameter level $(\mathrm{Mu})$. 
Table C-6: Equations of fitted response curve for Landscape_20_101

\begin{tabular}{|l|l|l|}
\hline Landscape_20_101 & Overall & Final epoch $=$ \\
& \\
& & $2.214 \times 10^{4}+5.246 \times 10^{3} \mathrm{Cr}-3.141 \times 10^{5} \mathrm{Mu}$ \\
& $+1.485 \times 10^{6} \mathrm{Mu}^{2}-2.285 \times 10^{6} \mathrm{Mu}^{3}$ \\
& $-5.009 \times 10^{4}(\mathrm{Cr} * \mathrm{Mu})+1.196 \times 10^{5}\left(\mathrm{Cr} * \mathrm{Mu}^{2}\right)$ \\
\hline
\end{tabular}

Crossover parameter level (Cr), Mutation parameter level (Mu). 


\section{Polynomial Regression Tables}

Table C-7: Polynomial Regression Tables for FN1R45 to FN6R45 and Landscape_20_101

\begin{tabular}{|c|c|c|}
\hline FN1R45 & Crossover & $100 \%$ \\
\hline \hline & Mutation & $8.78 \%$ \\
\hline \hline FN2R45 & Crossover & $0 \%$ \\
\hline \hline & Mutation & $25.45 \%$ \\
\hline \hline FN4R45 & Crossover & $33.23 \%$ \\
\hline \hline FN5R45 & Mutation & $12.36 \%$ \\
\hline & Crossover & $0 \%$ \\
\hline \hline FN6R45 & Mutation & $35.30 \%$ \\
\hline & Crossover & $39.17 \%$ \\
\hline \hline Landscape_20_101 & Crossover & $0 \%$ \\
\hline & Mutation & $18.93 \%$ \\
\hline
\end{tabular}

